\title{
A Labelled Sequent Calculus for BBI: Proof Theory and Proof Search
}

\author{
Zhé Hóu, Alwen Tiu and Rajeev Goré \\ Research School of Computer Science \\ The Australian National University \\ Canberra, ACT 0200, Australia
}

\begin{abstract}
We present a labelled sequent calculus for Boolean BI (BBI), a classical variant of the logic of Bunched Implication. The calculus is simple, sound, complete, and enjoys cut-elimination. We show that all the structural rules in the calculus, i.e., those rules that manipulate labels and ternary relations, can be localised around applications of certain logical rules, thereby localising the handling of these rules in proof search. Based on this, we demonstrate a free variable calculus that deals with the structural rules lazily in a constraint system. We propose a heuristic method to quickly solve certain constraints, and show some experimental results to confirm that our approach is feasible for proof search. Additionally, we show that different semantics for BBI and some axioms in concrete models can be captured by adding extra structural rules.
\end{abstract}

\section{Introduction}

The logic of bunched implications (BI) was introduced to reason about resources using additive connectives $\wedge, \vee, \rightarrow, T, \perp$, and multiplicative connectives $T^{*}, *,-*$ [14. Both parts are intuitionistic so BI is also Intuitionistic logic (IL) plus Lambek multiplicative logic (LM). Changing the additive part to classical logic gives Boolean BI (BBI). Replacing LM by multiplicative classical linear logic gives Classical BI (CBI). BI logics are closely related to separation logic [17, a logic for proving properties of programs. Thus, the semantics and proof theory of BI-logics, particularly for proof search, are important in computer science.

The ternary relational Kripke semantics of BBI-logics come in at least three different flavours: nondeterministic (ND), partial deterministic (PD), and total deterministic (TD) 9. These semantics give different logics w.r.t. validity, i.e., $B B I_{N D}, B B I_{P D}, B B I_{T D}$ respectively, and all are undecidable [39]. The purely syntactic proof theory of BBI also comes in three flavours: Hilbert calculi [1615, display calculi [1] and nested sequent calculi [15]. All are sound and complete w.r.t. the ND-semantics [5/115].

In between the relational semantics and the purely syntactic proof theory are the labelled tableaux of Larchey-Wendling and Galmiche which are sound and complete w.r.t. the PD-semantics [87]. They remark that "the adaptation of this tableaux system to $B B I_{T D}$ should be straightforward (contrary to $B B I_{N D}$ )" [10]. We return to these issues in Section 8

The structural rules of display calculi, especially the contraction rule on structures, are impractical for backward proof search. Nested sequents also face similar problems, and although Park et al. showed the admissibility of contraction in an improved nested sequent calculus, it contains other rules that explicitly contract structures. Their iterative deepening automated theorem prover for BBI based on nested sequents is terminating and incomplete for bounded depths, but complete and potentially non-terminating for an unbounded depth [15. The labelled tableaux of Larchey-Wendling and Galmiche compile all structural rules into PD-monoidal constraints, and are cut-fee complete for $\mathrm{BBI}_{\mathrm{PD}}$ using a potentially infinite counter-model construction [7. But effective proof search is only a "perspective" and is left as further work [7, page 2].

Surprisingly, many applications of BBI do not directly correspond to it's widely used non-deterministic semantics. For example, separation logic models are instances of partial deterministic models [9] while "memory models" for BBI are restricted to have indivisible units: "the empty memory cannot be split into non-empty pieces" 3. Our goal is to give a labelled proof system for BBI based upon the ND-semantics which easily extend to the PD- and TD-semantics, and also these other, more "practical", semantics.

Our labelled sequent calculus $L S_{B B I}$ for BBI adopts some features from existing labelled tableaux for BBI [8] and existing labelled sequent calculi for modal logics [12]. Unlike these calculi, some $L S_{B B I}$-rules contain substitutions on labels. From a proof-search perspective, labelled calculi are no better than display 
calculi since they require extra-logical rules to explicitly encode the frame conditions of the underlying (Kripke) semantics. Such rules, which we refer to simply as structural rules, are just as bad as display postulates for proof search since we may be forced to explore all potential models. As a step towards our goal, we show that the applications of these structural rules can be localised around logical rules. Thus these structural rules are only triggered by applications of logical rules, leading to a purely syntax-driven proof search procedure for $L S_{B B I}$.

Our work is novel from two perspectives. Compared to the labelled tableaux of Larchey-Wendling and Galmiche, we deal with the non-deterministic semantics of BBI, which they have flagged as a difficulty, and obtain a constructive cut-elimination procedure. Compared to the nested sequent calculus of Park et al., our calculus is much simpler, and generally gives much shorter proofs. Note that Park et al. actually gave a labelled variant of their nested sequent calculus, with the same logical rules as ours. However, their structural rules are still just notational variants of the original ones, which are lengthy and do not use ternary relations. We also show that adding certain structural rules to $L S_{B B I}$ allows us to obtain cut-free labelled calculi for all the other semantics mentioned above.

The rest of the paper is organized as follows. In Section 2, we present the semantics of BBI, following [9], and our labelled sequent calculus $L S_{B B I}$. We show that $L S_{B B I}$ is sound with respect to the semantics, and it is complete indirectly via a Hilbert system for BBI [5] which is already shown complete. In Section 3 , we prove some important proof theoretic properties of $L S_{B B I}$ : invertibility of inference rules, admissibility of contraction, and more importantly, cut-elimination. In Section 4 we discuss a permutation result for inference rules, allowing us to isolate applications of structural rules during proof search. In Section 5. we describe how to reduce proof search to constraint solving in a free-variable sequent calculus. We give a heuristic method for solving the resulting constraint problem in Section 6 and report on experimental results in Section 7 Section 8 concludes the paper. Detailed proofs are available in the appendices.

\section{The Labelled Sequent Calculus for BBI}

The semantics of BBI is in Section 2.1 then we present our labelled calculus in Section 2.2. The soundness proof is outlined in Section 2.3, followed by the completeness proof in Section 2.4, in which the Hilbert system of BBI is used.

\subsection{Syntax and Semantics of BBI}

BBI formulae are defined inductively as follows, where $p$ is an atomic proposition, $\top^{*}, *,-*$ are the multiplicative unit, conjunction, and implication respectively:

$$
A::=p|\top| \perp|\neg A| A \vee A|A \wedge A| A \rightarrow A\left|\top^{*}\right| A * A \mid A-* A
$$

The labelled sequent calculus for BBI employs a ternary relation of worlds that is based on a nondeterministic monoid structure, a là Galmiche et al. [5].

A non-deterministic monoid is a triple $(\mathcal{M}, \circ, \epsilon)$ where $\mathcal{M}$ is a non-empty set, $\epsilon \in \mathcal{M}$ and $\circ: \mathcal{M} \times \mathcal{M} \rightarrow$ $\mathcal{P}(\mathcal{M})$. The extension of $\circ$ to $\mathcal{P}(\mathcal{M})$ uses $X \circ Y=\bigcup\{x \circ y: x \in X, y \in Y\}$. The following conditions hold in this monoid:

- Identity: $\forall a \in \mathcal{M} . \epsilon \circ a=\{a\}$

- Commutativity: $\forall a, b \in \mathcal{M} . a \circ b=b \circ a$

- Associativity: $\forall a, b, c \in \mathcal{M} . a \circ(b \circ c)=(a \circ b) \circ c$.

The ternary relation over worlds is defined by $\triangleright \subseteq \mathcal{M} \times \mathcal{M} \times \mathcal{M}$ such that $\triangleright(a, b, c)$ if and only if $c \in a \circ b$. Following Galmiche et al., we write $a, b \triangleright c$ instead of $\triangleright(a, b, c)$. We therefore have the following conditions for all $a, b, c, d \in \mathcal{M}$ :

- Identity: $\epsilon, a \triangleright b$ iff $a=b$

- Commutativity: $a, b \triangleright c$ iff $b, a \triangleright c$

- Associativity: If there exists $k$ s.t. $(a, k \triangleright d)$ and $(b, c \triangleright k)$ then there exists $l$ s.t. $(a, b \triangleright l)$ and $(l, c \triangleright d)$. 
Intuitively, the relation $x, y \triangleright z$ means that $z$ can be partitioned into two parts: $x$ and $y$. The identity condition can be read as every world can be partitioned into an empty world and itself. Commutativity captures that partitioning $z$ into $x$ and $y$ is the same as partitioning $z$ into $y$ and $x$. Finally, associativity means that if $z$ can be partitioned into $x$ and $y$, and $x$ can further be partitioned into $u$ and $v$, then all together $z$ consists of $u, v$ and $y$. Therefore there must exist an element $w$ which is the combination of $v$ and $y$, such that $w$ and $u$ form $z$. Note that since we do not restrict this monoid to be cancellable, $(x, y \triangleright x)$ does not imply $y=\epsilon$.

Let $(\mathcal{M}, \triangleright, \epsilon)$ be a relational frame and $v: \operatorname{Var} \rightarrow \mathcal{P}(\mathcal{M})$ be a valuation. A forcing relation "IF" between elements of $\mathcal{M}$ and BBI-formulae is defined as follows [5]:

$$
\begin{aligned}
& m \Vdash T^{*} \text { iff } m=\epsilon \quad m \Vdash P \text { iff } P \in V a r \text { and } m \in v(P) \\
& m \Vdash \perp \text { iff never } \quad m \Vdash A \vee B \text { iff } m \Vdash A \text { or } m \Vdash B \\
& m \Vdash \top \text { iff always } \quad m \Vdash A \wedge B \text { iff } m \Vdash A \text { and } m \Vdash B \\
& m \Vdash \neg A \text { iff } m \Vdash A \text { iff } m \Vdash A \rightarrow B \text { iff } m \Vdash A \text { or } m \Vdash B \\
& m \Vdash A * B \text { iff } \exists a, b .(a, b \triangleright m \text { and } a \Vdash A \text { and } b \Vdash B) \\
& m \Vdash A-* B \text { iff } \forall a, b .((m, a \triangleright b \text { and } a \Vdash A) \text { implies } b \Vdash B)
\end{aligned}
$$

A formula $A$ is true at $m \in \mathcal{M}$ if $m \Vdash A$ and is valid if $m \Vdash A$ for every $m \in \mathcal{M}$ in every model $(\mathcal{M}, \triangleright, \epsilon, v)$.

\subsection{The Labelled Sequent Calculus}

The inference rules of our labelled system $L S_{B B I}$ are shown in Figure 1, where we use $A$ to denote a formula, $w, x, y, z$ are in the set $L V$ ar of label variables, and $\epsilon$ is the label constant. We define a mapping $\rho:\{\epsilon\} \cup L V$ ar $\rightarrow \mathcal{M}$ from labels to worlds. Note that we overload the notation so that the empty world in the semantics and the label constant are both referred to as $\epsilon$, the ternary relation in the semantics and the calculus are both referred to as $\triangleright$. Therefore we impose the following condition on mappings from labels to worlds: $\forall \rho . \rho(\epsilon)=\epsilon$. We shall assume this condition implicitly in what follows.

A labelled formula $w: A$ means formula $A$ is true in world $\rho(w)$. A relational atom $(x, y \triangleright z)$ is interpreted as $\rho(x), \rho(y) \triangleright \rho(z)$ in the semantics. That is, a labelled formula $w: A$ is true iff $\rho(w) \Vdash A$, and a relational atom $(x, y \triangleright z)$ is true iff $\rho(x), \rho(y) \triangleright \rho(z)$ holds.

A sequent is of the form $\Gamma \vdash \Delta$, where $\Gamma$ and $\Delta$ are structures, the empty structure is $\emptyset_{a}$ and $\Gamma$ and $\Delta$ are multisets of labelled formulae and relational atoms, defined formally via:

$$
\begin{aligned}
& \Gamma::=\emptyset_{a}|w: A|(x, y \triangleright z) \mid \Gamma ; \Gamma \\
& \Delta::=w: A \mid \Delta ; \Delta
\end{aligned}
$$

Definition 1 (Sequent Validity). A sequent $\Gamma \vdash \Delta$ in $L S_{B B I}$ is valid if for all $(\mathcal{M}, \triangleright, \epsilon), v$ and $\rho$, if every member of $\Gamma$ is true then so is at least one member of $\Delta$.

Note that BBI-validity of a formula $A$ corresponds to validity of the sequent $\vdash x: A$, where $x$ is an arbitrary label. This notion of validity is also adopted in other work for BBI 915] and CBI [2. but is stronger than that used for BI [16, where a valid sequent is defined as one with a multiplicative unit on the left hand side. For example, the formula $T^{*}$ is valid in $\mathrm{BI}$, but it is not a valid in our definition because the sequent $\vdash x: T^{*}$ is not provable (although the sequent $\vdash \epsilon: T^{*}$ is provable). Translated to our setting, this would correspond to defining a valid formula as one which is true in the world $\epsilon$.

In our definition of sequents, the structural connective ";" in the antecedent means (additive) "and" whereas in the succedent it means (additive) "or". This is slightly different from the traditional sequent notation where "," is used as the structural connective. Our notation is consistent with sequent systems for the family of Bunched Implication (BI) logics, where ";" is the additive structural connective, and "," is used to denote the multiplicative structural connective. The multiplicative structural connective is not explicitly presented in our sequent notation, but as we shall see later, it is encoded implicitly in the relational atoms.

The formula introduced in the conclusion of each rule is the principal formula, and the relational atom introduced in the conclusion of each rule is the principal relational atom.

The semantics of $*$ involves an existential condition, so rules $* L$ and $* R$ incorporate existential and universal quantifiers respectively. Similarly, $-* L$ and $-* R$ incorporate universal and existential quantifiers 
Identity and Cut:

$$
\bar{\Gamma}_{\Gamma: P: P \vdash w: P ; \Delta}{ }^{i d} \quad \frac{\Gamma \vdash x: A ; \Delta \quad \Gamma^{\prime} ; x: A \vdash \Delta^{\prime}}{\Gamma ; \Gamma^{\prime} \vdash \Delta ; \Delta^{\prime}} \text { cut }
$$

\section{Logical Rules:}

$$
\begin{aligned}
& \overline{\Gamma ; w: \perp \vdash \Delta}^{\perp L} \quad \frac{\Gamma[\epsilon / w] \vdash \Delta[\epsilon / w]}{\Gamma ; w: \top^{*} \vdash \Delta} \top^{*}{ }_{L} \quad \frac{}{\Gamma \vdash w: \top ; \Delta}^{\top R} \quad \frac{}{\Gamma \vdash \epsilon: \top^{*} ; \Delta}{ }^{*} R \\
& \frac{\Gamma ; w: A ; w: B \vdash \Delta}{\Gamma ; w: A \wedge B \vdash \Delta} \wedge L \quad \frac{\Gamma \vdash w: A ; \Delta \quad \Gamma \vdash w: B ; \Delta}{\Gamma \vdash w: A \wedge B ; \Delta} \wedge R \\
& \frac{\Gamma \vdash w: A ; \Delta \quad \Gamma ; w: B \vdash \Delta}{\Gamma ; w: A \rightarrow B \vdash \Delta} \rightarrow L \quad \frac{\Gamma ; w: A \vdash w: B ; \Delta}{\Gamma \vdash w: A \rightarrow B ; \Delta} \rightarrow R \\
& \frac{(x, y \triangleright z) ; \Gamma ; x: A ; y: B \vdash \Delta}{\Gamma ; z: A * B \vdash \Delta} * L \quad \frac{(x, y \triangleright z) ; \Gamma ; x: A \vdash z: B ; \Delta}{\Gamma \vdash y: A-* B ; \Delta}-* R \\
& \frac{(x, y \triangleright z) ; \Gamma \vdash x: A ; z: A * B ; \Delta \quad(x, y \triangleright z) ; \Gamma \vdash y: B ; z: A * B ; \Delta}{(x, y \triangleright z) ; \Gamma \vdash z: A * B ; \Delta} * R \\
& \frac{(x, y \triangleright z) ; \Gamma ; y: A-* B \vdash x: A ; \Delta \quad(x, y \triangleright z) ; \Gamma ; y: A-* B ; z: B \vdash \Delta}{(x, y \triangleright z) ; \Gamma ; y: A-* B \vdash \Delta}-* L
\end{aligned}
$$

\section{Structural Rules:}

$$
\begin{array}{cc}
\frac{(y, x \triangleright z) ;(x, y \triangleright z) ; \Gamma \vdash \Delta}{(x, y \triangleright z) ; \Gamma \vdash \Delta} E & \frac{(u, w \triangleright z) ;(y, v \triangleright w) ;(x, y \triangleright z) ;(u, v \triangleright x) ; \Gamma \vdash \Delta}{(x, y \triangleright z) ;(u, v \triangleright x) ; \Gamma \vdash \Delta} A \\
\frac{(x, \epsilon \triangleright x) ; \Gamma \vdash \Delta}{\Gamma \vdash \Delta} U & \frac{(x, w \triangleright x) ;(y, y \triangleright w) ;(x, y \triangleright x) ; \Gamma \vdash \Delta}{(x, y \triangleright x) ; \Gamma \vdash \Delta} A_{C} \\
\frac{\left(\epsilon, w^{\prime} \triangleright w^{\prime}\right) ; \Gamma\left[w^{\prime} / w\right] \vdash \Delta\left[w^{\prime} / w\right]}{\left(\epsilon, w \triangleright w^{\prime}\right) ; \Gamma \vdash \Delta} E q_{1} & \frac{\left(\epsilon, w^{\prime} \triangleright w^{\prime}\right) ; \Gamma\left[w^{\prime} / w\right] \vdash \Delta\left[w^{\prime} / w\right]}{\left(\epsilon, w^{\prime} \triangleright w\right) ; \Gamma \vdash \Delta} E q_{2}
\end{array}
$$

\section{Side conditions:}

In $\top^{*} L, E q_{1}$ and $E q_{2}, w \neq \epsilon$.

In $* L$ and $-* R$, the labels $x$ and $y$ do not occur in the conclusion.

In $A$ and $A_{C}$, the label $w$ does not occur in the conclusion.

Fig. 1. The (cut-free) labelled sequent calculus $L S_{B B I}$ for Boolean BI.

respectively. Therefore, rules $* L$ and $-* R$ create a premise containing new relations, and the labels in the created relation must be fresh (except for the label of the principal formula). Rules $* R$ and $-* L$ create a premise using already existing relations from the conclusion. Further, in rules $A$ and $A_{C}$, the label $w$ must be fresh in the premise, as it represents a new partition of the original world.

In the rule $T^{*} L$, there is an operation of global substitution $[\epsilon / x]$ in the premise. A substitution $\Gamma[y / x]$ is defined in the usual way: replace every occurrence of $x$ in $\Gamma$ by $y$.

The additive rules $(\perp L, \top R, \wedge L, \wedge R, \rightarrow L, \rightarrow R)$ and the multiplicative rules $\left(\top^{*} L, \top^{*} R, * L, * R,-* L\right.$, $-* R$ ) respectively deal with the additive/ multiplicative connectives. The zero-premise rules are those with no premise $\left(i d, \perp L, \top R, \top^{*} R\right)$. Figure 2 shows an example derivation of $L S_{B B I}$.

Note that we start (at the bottom) by labelling the formula with an arbitrary world $a$. Since provability is preserved by substitutions of labels (Lemma 1), provability of $\vdash a: F$ implies provability of $\vdash w: F$, for any world $w$. Thus, if a formula is provable, then it is true in every world. 


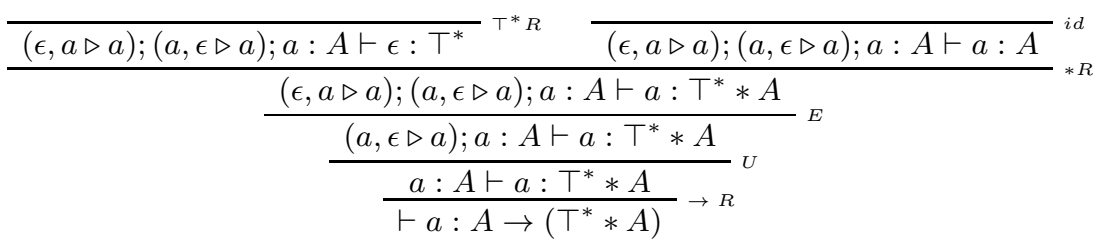

Fig. 2. An example derivation in $L S_{B B I}$.

\section{Axioms}

1. $A \rightarrow\left(\top^{*} * A\right)$

2. $\left(\top^{*} * A\right) \rightarrow A$

3. $(A * B) \rightarrow(B * A)$

4. $(A *(B * C)) \rightarrow((A * B) * C)$

\section{Deduction Rules}

$$
\begin{aligned}
& \frac{\vdash A \quad \vdash A \rightarrow B}{\vdash B} M P \frac{\vdash A \rightarrow C \quad \vdash B \rightarrow D}{\vdash(A * B) \rightarrow(C * D)} * \\
& \frac{\vdash A \rightarrow(B-* C)}{\vdash(A * B) \rightarrow C}-* 1 \quad \frac{\vdash(A * B) \rightarrow C}{\vdash A \rightarrow(B-* C)}-* 2
\end{aligned}
$$

Fig. 3. Some axioms and rules for the Hilbert system for BBI.

\subsection{Soundness}

The soundness proof reasons about the falsifiability of sequents, which is defined as follows.

Definition 2 (Sequent Falsifiability). A sequent $\Gamma \vdash \Delta$ in $L S_{B B I}$ is falsifiable if there exist some $(\mathcal{M}, \triangleright, \epsilon), v$ and $\rho$, such that every relational atom and labelled formula in $\Gamma$ is true and every labelled formula in $\Delta$ is false, where:

$w: A$ is true iff $\rho(w) \Vdash A$

$w: A$ is false iff $\rho(w) \forall A$

$(x, y \triangleright z)$ is true iff $\rho(x), \rho(y) \triangleright \rho(z)$ holds

Theorem 1 (Soundness). The labelled sequent calculus $L S_{B B I}$ is sound w.r.t. the Kripke semantics for $B B I$.

Proof. To prove the soundness of $L S_{B B I}$, we show that each rule preserves falsifiability upwards, as this is a more natural direction in terms of backward proof search. Therefore to prove that a rule is sound, we need to show that if the conclusion is falsifiable, then at least one of the premises is falsifiable (usually in the same choice of $v, \rho$, and $\mathcal{M}$ ). As the rules in $L S_{B B I}$ are designed based on the semantics, this is easy to verify. The details are in Appendix A.1

\subsection{Completeness}

We prove the completeness of $L S_{B B I}$ by showing that every derivation of a formula in the Hilbert system for BBI [5] can be mimicked in $L S_{B B I}$, possibly using cuts.

The Hilbert system for BBI consists of the axioms and rules for classical propositional logic for the additive fragment and additional axioms and rules for the multiplicative fragment. For the latter, we use the axiomatisation given in 5, and listed in Figure 3. We omit the axioms for classical propositional logic as they are standard, and can be found in, e.g., [18].

Theorem 2 (Completeness). The labelled sequent calculus $L S_{B B I}$ is complete w.r.t. the Kripke semantics for $B B I$. 
Proof. Given a derivation $\Pi$ of a formula $A$ in the Hilbert system for BBI, we show that one can construct an $L S_{B B I}$ derivation $\Pi^{\prime}$ of the sequent $\emptyset_{a} \vdash w: A$, for any label $w \neq \epsilon$. It is enough to show that each axiom and each rule of the Hilbert system can be derived. The derivations of the axioms in $L S_{B B I}$ are straightforward; we show here a non-trivial case in the derivation of the rules of the Hilbert system. Consider the rule $-* 1$ : Suppose $\Pi$ is the derivation:

$$
\begin{gathered}
\Pi_{1} \\
\frac{A \rightarrow(B-* C)}{(A * B) \rightarrow C}-* 1
\end{gathered}
$$

The $L S_{B B I}$ derivation $\Pi^{\prime}$ is in Figure 4, where $\Pi_{1}^{\prime}$ comes from $\Pi_{1}$ via the induction hypothesis, $\Pi_{2}$ is the upper derivation in Figure 4 and $\left.\Gamma=\left\{\left(w_{1}, w_{2} \triangleright w\right) ; w_{1}: A ; w_{2}: B\right\}\right)$.

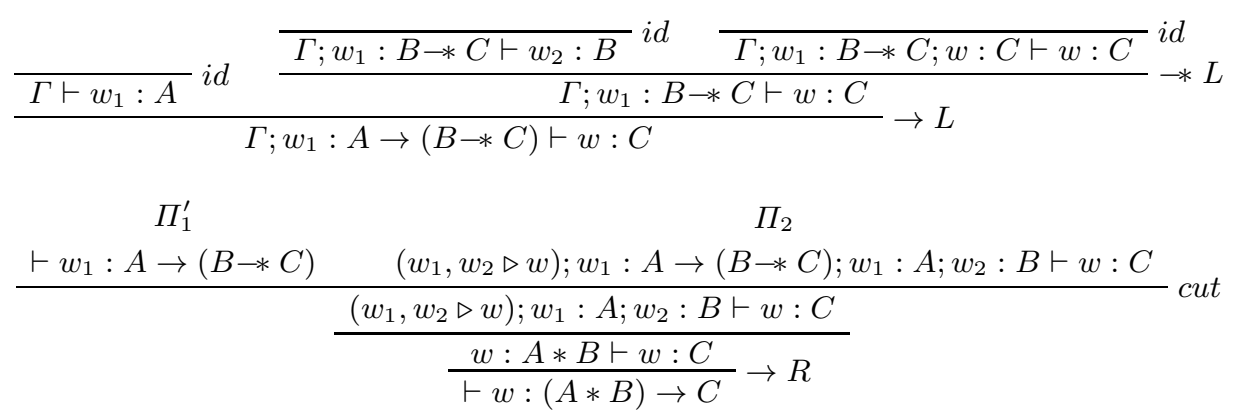

Fig. 4. A derivation of the rule $-* 1$ putting $\left.\Gamma=\left\{\left(w_{1}, w_{2} \triangleright w\right) ; w_{1}: A ; w_{2}: B\right\}\right)$.

Corollary 1 (Formula validity). A BBI formula $A$ is valid iff $\emptyset_{a} \vdash w: A$ is derivable in $L S_{B B I}$, for any arbitrary $w$.

Proof. Follows from the soundness and completeness proof. Since $w$ is arbitrary, $A$ is true at any world for any valuation $v$, mapping $\rho$, and monoid structure $(\mathcal{M}, \triangleright, \epsilon)$.

\section{Cut-elimination}

This section proves the cut-elimination theorem for our labelled sequent calculus. The general proof outlined here is similar to the cut-elimination proof for labelled systems for modal logic [12, i.e., we start by proving a substitution lemma for labels, followed by proving the invertibility of inference rules, weakening admissibility, and contraction admissibility, before proceeding to the main cut-elimination proof. As there are many case analyses in these proofs, we only outline the important parts here. More details are available in Appendix $\mathrm{A}$

Given a derivation $\Pi$, its height $h t(\Pi)$ is defined as the length of a longest branch in the derivation tree of $\Pi$.

The substitution lemma shows that provability is preserved under arbitrary substitutions of labels.

Lemma 1 (Substitution). If $\Pi$ is an $L S_{B B I}$ derivation for the sequent $\Gamma \vdash \Delta$ then there is an $L S_{B B I}$ derivation $\Pi^{\prime}$ of the sequent $\Gamma[y / x] \vdash \Delta[y / x]$ where every occurrence of label $x(x \neq \epsilon)$ is replaced by label $y$, such that $h t\left(\Pi^{\prime}\right) \leq h t(\Pi)$.

Proof. By induction on $h t(\Pi)$. We do case analyses on the last rule of $\Pi$. Most of the cases are similar to Negri's labelled calculus for modal logic [12, the only non-trivial cases are when the last rule is either $T^{*} L$, $E q_{1}$ or $E q_{2}$, and the labels $x$ or $y$ are used in the principal formula/relational atom. The full proof is in Appendix A.2.

Admissibility of weakening is proved by a simple induction on the height of derivations so we state the lemma sans proof. 
Lemma 2 (Weakening admissibility). If $\Gamma \vdash \Delta$ is derivable in $L S_{B B I}$, then for all structures $\Gamma^{\prime}$ and $\Delta^{\prime}$, the sequent $\Gamma ; \Gamma^{\prime} \vdash \Delta ; \Delta^{\prime}$ is derivable with the same height in $L S_{B B I}$.

Combining Lemma 1 and Lemma 2] we can replace a formula that is never used in a derivation by any structure. More supplementary lemmas related to weakening are listed in Appendix A.3.

Lemma 3 (Invertibility of rules). If $\Pi$ is a cut-free $L S_{B B I}$ derivation of the conclusion of a rule, then there is a cut-free $L S_{B B I}$ derivation for each premise, with height at most $h t(\Pi)$.

Proof. Most of the rules are trivially invertible. The proofs for the additive rules are similar to those for the additive rules from labelled calculi for modal logic or $G 3 c$ (cf. [13]) since the rules are the same. The slightly non-trivial cases for the rules involving substitutions of labels follow from Lemma 1, The proof is detailed in Appendix A.4.

The proof of the admissibility of contraction on additive formulae is similar to that for classical sequent calculus since the $L S_{B B I}$ rules for these connectives are the same. In the multiplicative rules, the principal formula is retained in the premise, so admissibility of contraction on multiplicative formulae follows trivially. We need to prove that contraction on relational atoms is admissible, as stated in the next lemma.

Lemma 4. For all structures $\Gamma, \Delta$, and ternary relations $(x, y \triangleright z)$ : if $\Pi$ a cut-free $L S_{B B I}$ derivation of $(x, y \triangleright z) ;(x, y \triangleright z) ; \Gamma \vdash \Delta$, then there is a cut-free $L S_{B B I}$ derivation $\Pi^{\prime}$ of $(x, y \triangleright z) ; \Gamma \vdash \Delta$ with $h t\left(\Pi^{\prime}\right) \leq h t(\Pi)$.

Proof. (Outline) Let $n=h t(\Pi)$. The proof is by induction on $n$. Most of structural rules only has one principal relational atom, so it is easy to show that contraction can permute through them.

The case for $A$ needs more care, as it involves two principal relations. If the two principal relations are different, then the admissibility of contraction follows similarly as above. But if the principal relations are a pair of identical relations, the situation is a bit tricky. The original derivation runs as follows.

$$
\begin{aligned}
& \Pi \\
& \frac{(x, w \triangleright x) ;(y, y \triangleright w) ;(x, y \triangleright x) ;(x, y \triangleright x) ; \Gamma \vdash \Delta}{(x, y \triangleright x) ;(x, y \triangleright x) ; \Gamma \vdash \Delta} A
\end{aligned}
$$

There is no obvious way to make this case admissible, and this is the reason we have a special case of the rule $A$, namely $A_{C}$. In the rule $A_{C}$, contraction is absorbed so that there is only one principal relation. The new derivation is as follows.

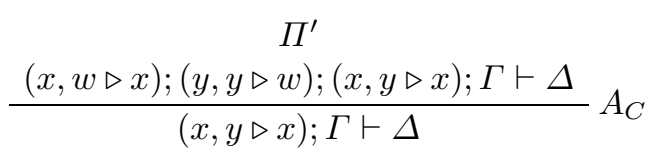

For $E q_{1}$ and $E q_{2}$, as the principal relation is carried to the premise (although some labels may be changed), so admissibility of contraction on those relations is obvious.

The admissibility of contraction on formulae are straightforward, the most of cases are analogous to the ones in Negri's labelled calculus for modal logic [12. For details please see Appendix A.5.

Lemma 5 (Contraction admissibility). If $\Gamma ; \Gamma \vdash \Delta ; \Delta$ is derivable in $L S_{B B I}$, then $\Gamma \vdash \Delta$ is derivable with the same height in $L S_{B B I}$.

\section{Cut Elimination Theorem}

We define the complexity of an application of the cut rule as $\left(|f|, h t\left(\Pi_{1}\right)+h t\left(\Pi_{2}\right)\right)$, where $|f|$ denotes the size of the cut formula (i.e., the number of connectives in the formula), and $h t\left(\Pi_{1}\right), h t\left(\Pi_{2}\right)$ are the heights of the derivations above the cut rule, the sum of them is call the cut height. If there are multiple branches in $\Pi_{1}$, then $h t\left(\Pi_{1}\right)$ shall be the height of the longest branch, similarly for $h t\left(\Pi_{2}\right)$. The strict ordering for both parts of the pair is $>$ on natural numbers.

Theorem 3 (Cut-elimination). If $\Gamma \vdash \Delta$ is derivable in $L S_{B B I}$, then it is also derivable without using the cut rule. 
Proof. By induction on the complexity of the proof in $L S_{B B I}$. We show that each application of cut can either be eliminated, or be replaced by one or more cut rules of less complexity. The argument for termination is similar to the cut-elimination proof for G3ip [13. We start to eliminate the topmost cut first, and repeat this procedure until there is no cut in the derivation. We first show that cut can be eliminated when the cut height is the lowest, i.e., at least one premise is of height 1 . Then we show that the cut height is reduced in all cases in which the cut formula is not principal in both premises of cut. If the cut formula is principal in both premises, then the cut is reduced to one or more cuts on smaller formulae or shorter derivations. Since atoms cannot be principal in logical rules, finally we can either reduce all cuts to the case where the cut formula is not principal in both premises, or reduce those cuts on compound formulae until their cut heights are minimal and then eliminate those cuts. The case analyses are shown in Appendix A.6. Here we only present one interesting case where the cut formula is principal in both premises, and the rules applied on each premise are $* R$ and $* L$ respectively.

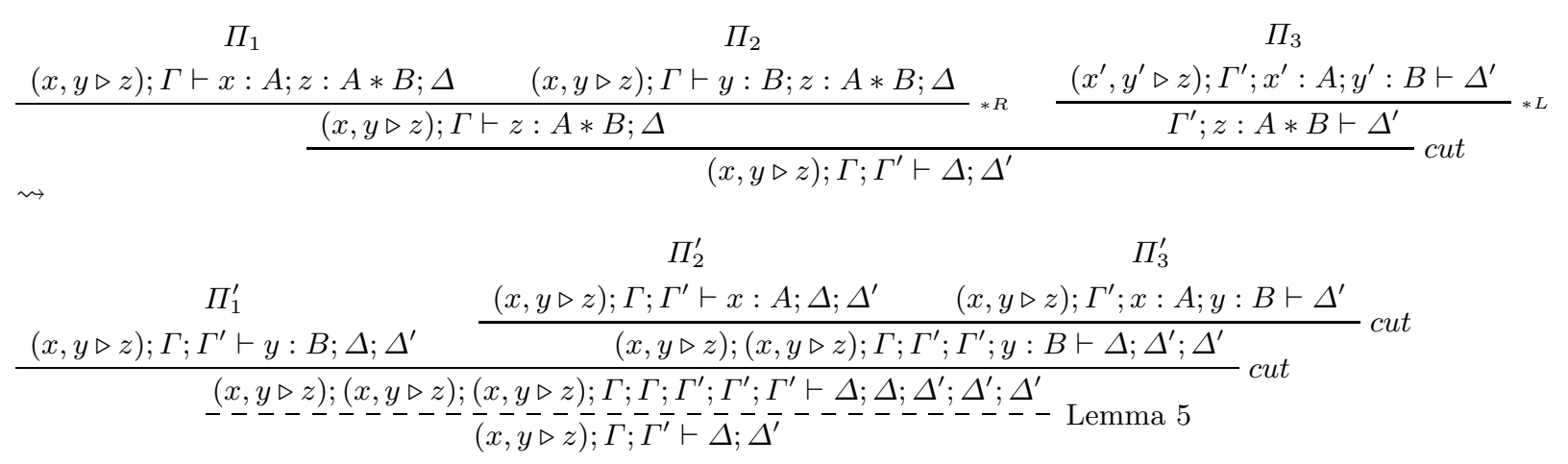

Fig. 5. The cut reduction for $*$ where the cut formula is principal in both premises.

The cut transformation in this case is given in Figure 5 . There, the derivation $\Pi_{1}^{\prime}$ (likewise, $\Pi_{2}^{\prime}$ ) is obtained by applying a cut to $\Pi_{1}$ (resp., $\Pi_{2}$ ) and the right premise of the original cut. We must also apply Lemma 5 to remove excess contexts.

\section{Localising structural rules}

As a first step towards designing an effective proof search procedure for $L S_{B B I}$, we need to restrict the use of structural rules.

We remark the fact that the structural rules in $L S_{B B I}$ can permute through all other rules except for $i d, T^{*} R, * R$, and $-* L$. We refer to these four rules as positive rules, and the rest logical rules in $L S_{B B I}$ as negative rules. The main reason is, all negative rules do not rely on the relational atoms. This is formalised in the following lemma, and proved in Appendix A.7.

Lemma 6. The structural rules in $L S_{B B I}$ can permute upwards through any negative rules in $L S_{B B I}$.

Then we design a more compact proof system where applications of structural rules are separated into a special entailment relation for relational atoms. We shall see in the next section that proof search in this proof system can be separated into two phases: guessing the shape of the proof tree, and deriving the relational atoms needed. The latter will be phrased in terms of a constraint system.

In this section we localise the structural rules in two steps: we first deal with $E q_{1}$ and $E q_{2}$, and then the other structural rules.

\subsection{Localising $E q_{1}$ and $E q_{2}$}

Allowing substitutions in a proof rule simplifies the cut-elimination proof for $L S_{B B I}$. However, for proof search, this creates a problem as $E q_{1}$ and $E q_{2}$ do not permute over certain rules that require matching 
of two labels (e.g., $* R$ or $-* L$ ). Our first intermediate proof system $L S_{B B I}^{e}$ aims to remove substitutions from $L S_{B B I}$. Instead, the equality between labels is captured via a special entailment relation. To define its inference rules, we first need a few preliminary definitions.

Let $r$ be an instance of a structural rule. We can view $r$ as a function that takes a set of relational atoms (in the conclusion of the rule) and outputs another set (in the premise). We shall write $r(\mathcal{G}, \theta)$, where $\mathcal{G}$ is the set of principal relational atoms and $\theta$ is a substitution, to denote the set of relational atoms introduced in the premise of an instance of $r$ with conclusion containing $\mathcal{G}$, and where the substitution used in the rule is $\theta$, which is the identity substitution in all cases except when $r$ is $E q_{1}$ or $E q_{2}$. Let $\sigma$ be a sequence of instances of structural rules $\left[r_{1}\left(\mathcal{G}_{1}, \theta_{1}\right) ; \cdots ; r_{n}\left(\mathcal{G}_{n}, \theta_{n}\right)\right]$. Given a set of relation atoms $\mathcal{G}$, the result of the application of $\sigma$ to $\mathcal{G}$, denoted by $\mathcal{S}(\mathcal{G}, \sigma)$, is defined inductively as follows:

$$
\mathcal{S}(\mathcal{G}, \sigma)=\left\{\begin{array}{lll}
\mathcal{G} & \text { if } & \sigma=[] \\
\mathcal{S}\left(\mathcal{G} \theta \cup r\left(\mathcal{G}^{\prime}, \theta\right), \sigma^{\prime}\right) & \text { if } & \mathcal{G}^{\prime} \subseteq \mathcal{G} \text { and } \sigma=\left[r\left(\mathcal{G}^{\prime}, \theta\right) ; \sigma^{\prime}\right] \\
\text { undefined } & & \text { otherwise }
\end{array}\right.
$$

Given a $\sigma=\left[r_{1}\left(\mathcal{G}_{1}, \theta_{1}\right) ; \cdots ; r_{n}\left(\mathcal{G}_{n}, \theta_{n}\right)\right]$, we denote with $\operatorname{subst}(\sigma)$ the composite substitution $\theta_{1} \circ \cdots \circ \theta_{n}$, where $t\left(\theta_{1} \circ \theta_{2}\right)$ means $\left(t \theta_{1}\right) \theta_{2}$.

Definition 3. Let $\mathcal{G}$ be a set of relational atoms. The entailment relation $\mathcal{G} \vdash_{E} u=v$ holds iff there exists a sequence $\sigma$ of $E q_{1}$ or $E q_{2}$ structural rules such that $\mathcal{S}(\mathcal{G}, \sigma)$ is defined, and $u \theta=v \theta$, where $\theta=\operatorname{subst}(\sigma)$.

We now define the proof system $L S_{B B I}^{e}$ as $L S_{B B I} \backslash\left\{E q_{1}, E q_{2}\right\}$ (i.e., $L S_{B B I}$ without rules $\left.E q_{1}, E q_{2}\right)$ where certain rules modified according to Figure 6.

$$
\begin{aligned}
& \frac{\mathcal{G} \vdash_{E}\left(w_{1}=w_{2}\right)}{\Gamma ; w_{1}: P \vdash w_{2}: P ; \Delta}{ }_{i d} \quad \frac{(\epsilon, w \triangleright \epsilon) ; \Gamma \vdash \Delta}{\Gamma ; w: T^{*} \vdash \Delta} T^{*} L \quad \frac{\mathcal{G} \vdash_{E}(w=\epsilon)}{\Gamma \vdash w: \top^{*} ; \Delta} T^{*} R \\
& \frac{\left(x, y \triangleright z^{\prime}\right) ; \Gamma \vdash x: A ; z: A * B ; \Delta \quad\left(x, y \triangleright z^{\prime}\right) ; \Gamma \vdash y: B ; z: A * B ; \Delta \quad \mathcal{G} \vdash_{E}\left(z=z^{\prime}\right)}{\left(x, y \triangleright z^{\prime}\right) ; \Gamma \vdash z: A * B ; \Delta}{ }_{*} \\
& \frac{\left(x, y^{\prime} \triangleright z\right) ; \Gamma ; y: A-* B \vdash x: A ; \Delta \quad\left(x, y^{\prime} \triangleright z\right) ; \Gamma ; y: A-* B ; z: B \vdash \Delta \quad \mathcal{G} \vdash_{E}\left(y=y^{\prime}\right)}{\left(x, y^{\prime} \triangleright z\right) ; \Gamma ; y: A-* B \vdash \Delta}-* \\
& \frac{(u, w \triangleright z) ;(y, v \triangleright w) ;(x, y \triangleright z) ;\left(u, v \triangleright x^{\prime}\right) ; \Gamma \vdash \Delta \quad \mathcal{G} \vdash_{E}\left(x=x^{\prime}\right)}{(x, y \triangleright z) ;\left(u, v \triangleright x^{\prime}\right) ; \Gamma \vdash \Delta}{ }_{A} \\
& \frac{\left(x, w \triangleright x^{\prime}\right) ;(y, y \triangleright w) ;\left(x, y \triangleright x^{\prime}\right) ; \Gamma \vdash \Delta \quad \mathcal{G} \vdash_{E}\left(x=x^{\prime}\right)}{\left(x, y \triangleright x^{\prime}\right) ; \Gamma \vdash \Delta}{ }^{A_{C}}
\end{aligned}
$$

$\mathcal{G}$ is the set of relational atoms on the left hand side of the conclusion sequent.

Fig. 6. The changed rules in $L S_{B B I}^{e}$.

Note that the new $\top^{*} L$ rule does not modify any labels, instead, the relational atom $(\epsilon, w \triangleright \epsilon)$ in the premise ensures that the derivability of $(w=\epsilon)$ is preserved. The point of this intermediate step is to avoid label substitutions in the proof system.

Theorem 4. A sequent $\Gamma \vdash \Delta$ is derivable in $L S_{B B I}$ if and only if it is derivable in $L S_{B B I}^{e}$.

Proof. (Outline) One direction, from $L S_{B B I}^{e}$ to $L S_{B B I}$ is straightforward, as $\vdash_{E}$ is essentially just a sequence of applications of $E q_{1}$ and $E q_{2}$. The other direction can be proved by showing that $E q_{1}$ and $E q_{2}$ are admissible in $L S_{B B I}^{e}$. A more detailed proof is given in Appendix A.8, A.9, for soundness and completeness respectively. 


\subsection{Localising the rest of the structural rules}

As a second step, we isolate the rest structural rules into a separate entailment relation, as we did with $E q_{1}$ and $E q_{2}$.

Definition 4 (Relation Entailment $\vdash_{R}$ ). The entailment relation $\vdash_{R}$ has the following two forms:

1. $\mathcal{G} \vdash_{R}\left(w_{1}=w_{2}\right)$ is true iff there is a sequence $\sigma$ of $E, U, A, A_{C}$ applications so that $\mathcal{S}(\mathcal{G}, \sigma) \vdash_{E}\left(w_{1}=\right.$ $\left.w_{2}\right)$.

2. $\mathcal{G} \vdash_{R}\left(w_{1}, w_{2} \triangleright w_{3}\right)$ is true iff there is a sequence $\sigma$ of $E, U, A, A_{C}$ applications so that $\left(w_{1}^{\prime}, w_{2}^{\prime} \triangleright w_{3}^{\prime}\right) \in$ $\mathcal{S}(\mathcal{G}, \sigma)$ and the following hold: $\mathcal{S}(\mathcal{G}, \sigma) \vdash_{E}\left(w_{1}=w_{1}^{\prime}\right), \mathcal{S}(\mathcal{G}, \sigma) \vdash_{E}\left(w_{2}=w_{2}^{\prime}\right)$, and $\mathcal{S}(\mathcal{G}, \sigma) \vdash_{E}\left(w_{3}=w_{3}^{\prime}\right)$.

The entailment $\vdash_{R}$ is stronger than $\vdash_{E}$. For example, if $\mathcal{G}$ only contains $(x, \epsilon \triangleright y)$, then $\mathcal{G} \nvdash_{E}(x=y)$; but $\mathcal{G} \vdash_{R}(x=y)$ by applying $E$ to obtain $(\epsilon, x \triangleright y)$, then apply $E q_{1}$ or $E q_{2}$ on the new relational atom.

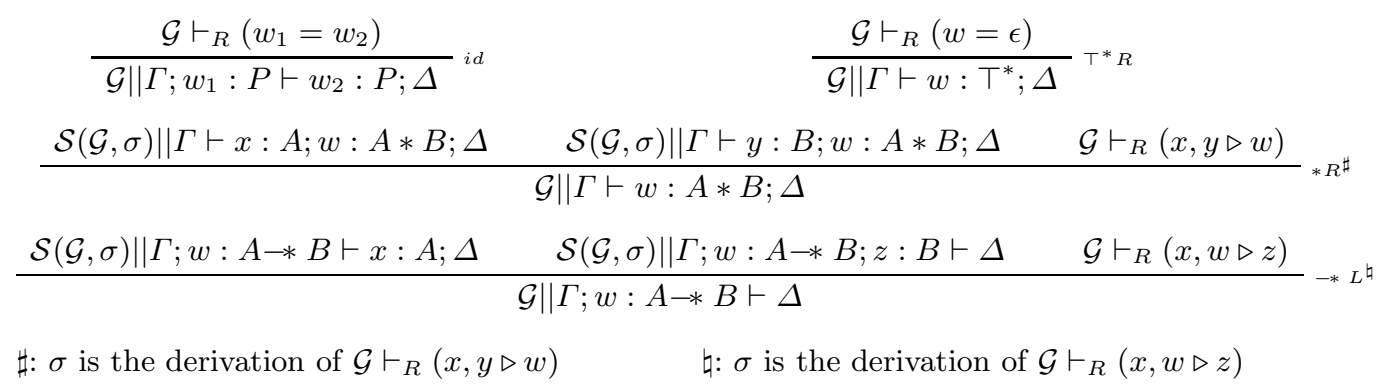

Fig. 7. Changed rules in $L S_{B B I}^{s f}$.

The changed rules in the second intermediate system $L S_{B B I}^{s f}$ is given in Figure 7 where we use a slightly different notation for sequents. We write $\mathcal{G} \| \Gamma \vdash \Delta$ to emphasize that the left hand side of a sequent is partitioned into two parts: $\mathcal{G}$, which contains only relational atoms, and $\Gamma$, which contains only labelled formulae.

The following is an immediate result, the proof is divided in two parts for soundness and completeness, detailed in Appendix A.11 and A.12 respectively.

Theorem 5. A sequent $\Gamma \vdash \Delta$ is derivable in $L S_{B B I}^{e}$ if and only if it is derivable in $L S_{B B I}^{s f}$.

\section{$5 \quad$ Mapping proof search to constraint solving}

We now consider a proof search strategy for $L S_{B B I}^{s f}$. As we have isolated all the structural rules into the entailment relation $\vdash_{R}$, proof search in $L S_{B B I}^{s f}$ consists of guessing the shape of the derivation tree, and then checking that each entailment $\vdash_{R}$ can be proved. The latter involves guessing a splitting of labels in the $* R$ and $-* L$ rules which also satisfies the equality constraints in the $i d$ and $T^{*} R$ rules. We formalise this via a symbolic proof system, where splitting and equality are handled lazily, via the introduction of free variables which are essentially existential variables (or logic variables) that must be instantiated to concrete labels satisfying all the entailment constraints in the proof tree, for a symbolic derivation to be sound.

Free variables are denoted by $\mathbf{x}, \mathbf{y}$ and $\mathbf{z}$. We use $\mathbf{u}, \mathbf{v}, \mathbf{w}$ to denote either labels or free variables, and $a, b, c$ are ordinary labels. A symbolic sequent is just a sequent but possibly with occurrences of free variables in place of labels. We shall sometimes refer to the normal (non-symbolic) sequent as a ground sequent to emphasize the fact that it contains no free variables. The symbolic proof system $F V L S_{B B I}$ is given in Figure 8. The rules are mostly similar to $L S_{B B I}^{s f}$, but lacking the entailment relations $\vdash_{R}$. Instead, new free variables are introduced when applying $* R$ and $-* L$ backward. Notice also that in $F V L S_{B B I}$, the $* R$ and $-* L$ rules do not compute the set $\mathcal{S}(\mathcal{G}, \sigma)$. So the relational atoms in $F V L S_{B B I}$ are those that are created by $* L,-* R, \top^{*} L$. In the following, given a derivation in $F V L S_{B B I}$, we shall assume that the free variables 


\section{Initial Sequent:}

$$
{\overline{\mathcal{G}} \| \Gamma ; \mathbf{w}_{1}: P \vdash \mathbf{w}_{2}: P ; \Delta}^{i d}
$$

\section{Logical Rules:}

$$
\begin{aligned}
& \overline{\mathcal{G} \| \Gamma ; \mathbf{w}: \perp \vdash \Delta}^{\perp L} \quad \overline{\mathcal{G} \| \Gamma \vdash \mathbf{w}: \top ; \Delta}^{\top R} \\
& \frac{\mathcal{G} ;(\epsilon, \mathbf{w} \triangleright \epsilon) \| \Gamma \vdash \Delta}{\mathcal{G} \| \Gamma ; \mathbf{w}: \top^{*} \vdash \Delta} T^{*}{ }_{L} \quad \frac{}{\mathcal{G} \| \Gamma \vdash \mathbf{w}: \top^{*} ; \Delta}{ }^{* *}{ }_{R} \\
& \frac{\mathcal{G} \| \Gamma ; \mathbf{w}: A ; \mathbf{w}: B \vdash \Delta}{\mathcal{G} \| \Gamma ; \mathbf{w}: A \wedge B \vdash \Delta} \wedge L \quad \frac{\mathcal{G}\|\Gamma \vdash \mathbf{w}: A ; \Delta \quad \mathcal{G}\| \Gamma \vdash \mathbf{w}: B ; \Delta}{\mathcal{G} \| \Gamma \vdash \mathbf{w}: A \wedge B ; \Delta} \wedge R \\
& \frac{\mathcal{G}\|\Gamma \vdash \mathbf{w}: A ; \Delta \quad \mathcal{G}\| \Gamma ; \mathbf{w}: B \vdash \Delta}{\mathcal{G} \| \Gamma ; \mathbf{w}: A \rightarrow B \vdash \Delta} \rightarrow L \quad \frac{\mathcal{G} \| \Gamma ; \mathbf{w}: A \vdash \mathbf{w}: B ; \Delta}{\mathcal{G} \| \Gamma \vdash \mathbf{w}: A \rightarrow B ; \Delta} \rightarrow R \\
& \frac{\mathcal{G} ;(a, b \triangleright \mathbf{w}) \| \Gamma ; a: A ; b: B \vdash \Delta}{\mathcal{G} \| \Gamma ; \mathbf{w}: A * B \vdash \Delta}{ }_{* L^{\dagger}} \quad \frac{\mathcal{G} ;(a, \mathbf{w} \triangleright c) \| \Gamma ; a: A \vdash c: B ; \Delta}{\mathcal{G} \| \Gamma \vdash \mathbf{w}: A-* B ; \Delta}{ }_{-* R^{\ddagger}} \\
& \frac{\mathcal{G}\|\Gamma \vdash \mathbf{x}: A ; \mathbf{w}: A * B ; \Delta \quad \mathcal{G}\| \Gamma \vdash \mathbf{y}: B ; \mathbf{w}: A * B ; \Delta}{\mathcal{G} \| \Gamma \vdash \mathbf{w}: A * B ; \Delta} * R^{\sharp} \\
& \frac{\mathcal{G}\|\Gamma ; \mathbf{w}: A-* B \vdash \mathbf{x}: A ; \Delta \quad \mathcal{G}\| \Gamma ; \mathbf{w}: A-* B ; \mathbf{z}: B \vdash \Delta}{\mathcal{G} \| \Gamma ; \mathbf{w}: A-* B \vdash \Delta}-* L^{\natural}
\end{aligned}
$$

$\dagger: a$ and $b$ must be fresh in $* L \quad \ddagger: a$ and $c$ must be fresh in $-* R$ $\sharp$ : $\mathbf{x}$ and $\mathbf{y}$ are new free variables in $* R \quad \sharp$ : $\mathbf{x}$ and $\mathbf{z}$ are new free variables in $-* L$

Fig. 8. Labelled Sequent Calculus $F V L S_{B B I}$ for Boolean BI.

that are created in different branches of the derivation are pairwise distinct. We shall sometimes refer to a derivation in $F V L S_{B B I}$ simply as a symbolic derivation.

An equality constraint is an expression of the form $\mathcal{G} \vdash_{R}^{?}(\mathbf{u}=\mathbf{v})$, and a relational constraint is an expression of the form $\mathcal{G} \vdash_{R}^{\text {? }}(\mathbf{u}, \mathbf{v} \triangleright \mathbf{w})$. In both cases, we refer to $\mathcal{G}$ as the left hand side of the constraints, and $(\mathbf{u}=\mathbf{v})$ and $(\mathbf{u}, \mathbf{v} \triangleright \mathbf{w})$ as the right hand side. Constraints are ranged over by $\mathfrak{c}, \mathfrak{c}^{\prime}, \mathfrak{c}_{1}, \mathfrak{c}_{2}$, etc. Given a constraint $\mathfrak{c}$, we write $\mathcal{G}(\mathfrak{c})$ for the left hand side of $\mathfrak{c}$. A constraint system is just a set of constraints. We write $\mathcal{G} \vdash{ }_{R}^{?} C$ for either an equality or relational constraint. We write $f v(\mathfrak{c})$ to denote the set of free variables in $\mathfrak{c}$, and $f v(\mathcal{C})$ to denote the set of free variables in a set of constraints $\mathcal{C}$.

Definition 5 (Constraint systems). A constraint system is a pair $(\mathcal{C}, \preceq)$ of a set of constraints and a well-founded partial order on elements of $\mathcal{C}$ satisfying Monotonicity: $\mathfrak{c}_{1} \preceq \mathfrak{c}_{2}$ implies $\mathcal{G}\left(\mathfrak{c}_{1}\right) \subseteq \mathcal{G}\left(\mathfrak{c}_{2}\right)$. It is well-formed if it also satisfies Unique variable origin: $\forall \mathbf{x}$ in $\mathcal{C}$, there exists a unique minimum (w.r.t. $\preceq$ ) constraint $\mathfrak{c}(\mathbf{x})=\mathcal{G}_{x} \vdash_{R}^{?}(\mathbf{u}, \mathbf{v} \triangleright \mathbf{w})$ s.t. $\mathbf{x}$ occurs in $(\mathbf{u}, \mathbf{v} \triangleright \mathbf{w})$, but not in $\mathcal{G}_{x}$, and $\mathbf{x}$ does not occur in any $\mathfrak{c}^{\prime}$ where $\mathfrak{c}^{\prime} \preceq \mathfrak{c}(\mathbf{x})$. Such $a \mathfrak{c}(\mathbf{x})$ is the origin of $\mathbf{x}$.

From now on, we shall denote with $\mathfrak{c}(\mathbf{x})$ the constraint where $\mathbf{x}$ originates from, as defined in the above definition. We use the letter $\mathbb{C}$ to range over constraint systems.

We write $\mathfrak{c}_{i} \prec \mathfrak{c}_{j}$ when $\mathfrak{c}_{i} \preceq \mathfrak{c}_{j}$ and $\mathfrak{c}_{i} \neq \mathfrak{c}_{j}$. Further, we define a direct successor relation $\lessdot$ as follows: $\mathfrak{c}_{i} \lessdot \mathfrak{c}_{j}$ iff $\mathfrak{c}_{i} \prec \mathfrak{c}_{j}$ and there does not exist any $\mathfrak{c}_{k}$ such that $\mathfrak{c}_{i} \prec \mathfrak{c}_{k} \prec \mathfrak{c}_{j}$

During proof search, associated constraints are generated as follows.

Definition 6. To a given symbolic derivation $\Pi$, we associate a set of constraints $\mathcal{C}(\Pi)$ as follows where the lowest rule instance of $\Pi$ is: 


$$
\begin{array}{ll}
\text { id } & \mathcal{C}(\Pi)=\left\{\mathcal{G} \vdash_{R}^{?}\left(\mathbf{w}_{1}=\mathbf{w}_{2}\right)\right\} \\
T^{*} R & \mathcal{C}(\Pi)=\left\{\mathcal{G} \vdash_{R}^{?}(\mathbf{w}=\epsilon)\right\} \\
* R & \mathcal{C}(\Pi)=\mathcal{C}\left(\Pi_{1}\right) \cup \mathcal{C}\left(\Pi_{2}\right) \cup\left\{\mathcal{G} \vdash_{R}(\mathbf{x}, \mathbf{y} \triangleright \mathbf{w})\right\} \text { where the left premise derivation is } \Pi_{1} \text { and } \\
& \text { the right-premise derivation is } \Pi_{2} \\
-* L & \mathcal{C}(\Pi)=\mathcal{C}\left(\Pi_{1}\right) \cup \mathcal{C}\left(\Pi_{2}\right) \cup\left\{\mathcal{G} \vdash_{R}^{?}(\mathbf{x}, \mathbf{w} \triangleright \mathbf{y})\right\} \text { where the left premise derivation is } \Pi_{1} \text { and } \\
& \text { the right-premise derivation is } \Pi_{2} \\
& \text { If } \Pi \text { ends with any other rule, with premise derivations }\left\{\Pi_{1}, \ldots, \Pi_{n}\right\}, \text { then } \mathcal{C}(\Pi)= \\
& \mathcal{C}\left(\Pi_{1}\right) \cup \cdots \cup \mathcal{C}\left(\Pi_{n}\right) .
\end{array}
$$

Each constraint $\mathfrak{c} \in \mathcal{C}(\Pi)$ corresponds to a rule instance $r(\mathfrak{c})$ in $\Pi$ where $\mathfrak{c}$ is generated. The ordering of the rules in the derivation tree of $\Pi$ then naturally induces a partial order on $\mathcal{C}(\Pi)$. That is, let $\preceq^{\Pi}$ be an ordering on $\mathcal{C}(\Pi)$ defined as follows: $\mathfrak{c}_{1} \preceq^{\Pi} \mathfrak{c}_{2}$ iff the conclusion of $r\left(\mathfrak{c}_{1}\right)$ appears in the path from the root sequent to the conclusion of $r\left(\mathfrak{c}_{2}\right)$. Then obviously $\preceq^{\Pi}$ is a partial order.

The following property of $\mathcal{C}(\Pi)$ is easy to verify.

Lemma 7. Let $\Pi$ is a symbolic derivation. Then $\left(\mathcal{C}(\Pi), \preceq^{\Pi}\right)$ is a constraint system. Moreover, if the root sequent is ground, then $\left(\mathcal{C}(\Pi), \preceq^{\Pi}\right)$ is well-formed.

Given a symbolic derivation $\Pi$, we define $\mathbb{C}(\Pi)$ as the constraint system $\left(\mathcal{C}(\Pi), \preceq^{\Pi}\right)$ as defined above.

A consequence of Lemma 7 is that if $\mathbb{C}(\Pi) \neq\{\}$, then there exists a minimum constraint $\mathfrak{c}$, w.r.t. the partial order $\preceq^{\Pi}$, such that $\mathcal{G}(\mathfrak{c})$ is ground.

We now define what it means for a constraint system to be solvable. This is a bit complicated, because we need to capture that (ternary) relational atoms created by the solution need to be accumulated across different constraints, in order to guarantee soundness of $F V L S_{B B I}$. A free-variable substitution $\theta$ is a mapping from free variables to free-variables or labels with finite domain. We denote with $\operatorname{dom}(\theta)$ the domain of $\theta$. Given $\theta$ and a set $V$ of free variables, $\theta \uparrow V$ is the substitution obtained from $\theta$ by restricting the domain to $V$, i.e.,

$$
\mathbf{x}(\theta \uparrow V)= \begin{cases}\mathbf{x} \theta & \text { if } \mathbf{x} \in V \\ \mathbf{x} & \text { otherwise. }\end{cases}
$$

Given $\theta$ and $\theta^{\prime}$ such that $\operatorname{dom}\left(\theta^{\prime}\right) \subseteq \operatorname{dom}(\theta)$, we define $\theta \backslash \theta^{\prime}$ as the substitution:

$$
\mathbf{x}\left(\theta \backslash \theta^{\prime}\right)= \begin{cases}\mathbf{x} \theta \text { if } \mathbf{x} \notin \operatorname{dom}\left(\theta^{\prime}\right) \\ \mathbf{x} \text { otherwise. }\end{cases}
$$

Definition 7 (Simple constraints and their solutions). A constraint $\mathfrak{c}$ is simple if its left hand side $\mathcal{G}(\mathfrak{c})$ contains no free variables. A solution $(\theta, \sigma)$ to a simple constraint $\mathfrak{c}$ is a substitution $\theta$ and a sequence $\sigma$ of structural rules such that:

- If $\mathfrak{c}$ is $\mathcal{G} \vdash_{R}^{?}(\mathbf{u}=\mathbf{v})$ then $\sigma$ is a derivation of $\mathcal{G} \vdash_{R}(\mathbf{u} \theta=\mathbf{v} \theta)$.

- If $\mathfrak{c}$ is $\mathcal{G} \vdash_{R}^{?}(\mathbf{u}, \mathbf{v} \triangleright \mathbf{w})$ then $\sigma$ is a derivation of $\mathcal{G} \vdash_{R}(\mathbf{u} \theta, \mathbf{v} \theta \triangleright \mathbf{w} \theta)$.

The minimum constraints of a well-formed constraint system are simple.

Definition 8 (Restricting a constraint system). Let $\mathbb{C}=(\mathcal{C}, \preceq)$ be a well-formed constraint system, and $\mathfrak{c}$ be a minimum (simple) constraint in $\mathbb{C}$. Let $(\theta, \sigma)$ be a solution to $\mathfrak{c}$ and $\mathcal{G}^{\prime}=\mathcal{S}(\mathcal{G}(\mathfrak{c}), \sigma)$. Define a function $f$ on constraints:

$$
f\left(\mathfrak{c}^{\prime}\right)= \begin{cases}\left(\mathcal{G}^{\prime} \cup \mathcal{G} \theta \vdash{ }_{R}^{?} C \theta\right) & \text { if } \mathfrak{c}^{\prime}=\left(\mathcal{G} \vdash{ }_{R}^{?} C\right) \in \mathcal{C} \backslash\{\mathfrak{c}\} \text { and } \mathfrak{c} \preceq \mathfrak{c}^{\prime}, \\ \mathfrak{c}^{\prime} & \text { otherwise. }\end{cases}
$$

The restriction of $\mathbb{C}$ by $(\mathfrak{c}, \theta, \sigma)$, written $\mathbb{C} \uparrow(\mathfrak{c}, \theta, \sigma)$, is the pair $\left(\mathcal{C}^{\prime}, \preceq^{\prime}\right)$, where (1) $\mathcal{C}^{\prime}=\left\{f\left(\mathfrak{c}^{\prime}\right) \mid \mathfrak{c}^{\prime} \in \mathcal{C} \backslash\{\mathfrak{c}\}\right\}$ and (2) $f\left(\mathfrak{c}_{1}\right) \preceq$ f $f\left(\mathfrak{c}_{2}\right)$ iff $\mathfrak{c}_{1} \preceq \mathfrak{c}_{2}$.

Lemma 8. The pair $\mathbb{C} \uparrow(\mathfrak{c}, \theta, \sigma)$ as defined in Definition 8 is a well-formed constraint system.

Definition 9 (Solution to a well-formed constraint system). Let $\mathbb{C}=\left(\left\{\mathfrak{c}_{1}, \ldots, \mathfrak{c}_{n}\right\}, \preceq\right)$ be a wellformed constraint system. A solution $\left(\theta,\left\{\sigma_{1}, \ldots, \sigma_{n}\right\}\right)$ to $\mathbb{C}$ is a substitution and a set of sequences of structural rules, such that: 
If $n=0$ then $\left(\theta,\left\{\sigma_{1}, \ldots, \sigma_{n}\right\}\right)$ is trivially a solution.

If $n \geq 1$ then there must exist some minimum (simple) constraint in $\mathbb{C}$. For any minimum constraint $\mathfrak{c}_{i}$, let $\theta_{i}=\theta \uparrow f v\left(\mathfrak{c}_{i}\right)$, then $\left(\theta_{i}, \sigma_{i}\right)$ is a solution to $\mathfrak{c}_{i}$, and $\left(\theta \backslash \theta_{i},\left\{\sigma_{1}, \ldots, \sigma_{n}\right\} \backslash \sigma_{i}\right)$ is a solution to $\mathbb{C} \uparrow\left(\mathfrak{c}_{i}, \theta_{i}, \sigma_{i}\right)$.

In Definition 9, suppose a constraint system $\mathbb{C}=\left(\left\{\mathfrak{c}_{1}, \cdots, \mathfrak{c}_{n}\right\}, \preceq\right)$ has a solution $\left(\theta,\left\{\sigma_{1}, \cdots, \sigma_{n}\right\}\right)$, then for each constraint $\mathfrak{c}_{i}$ in $\mathbb{C}$, let $\mathfrak{c}_{i}^{\prime}$ be the simple constraint obtained from $\mathfrak{c}_{i}$ in the process of restricting $\mathbb{C}$, there is a solution $\left(\theta_{i}, \sigma_{i}\right)$ for $\mathfrak{c}_{i}^{\prime}$, where $\theta_{i}$ is a restriction on $\theta$ that contains the free variables in $\mathfrak{c}_{i}^{\prime}$, and $\sigma_{i} \in\left\{\sigma_{1}, \cdots, \sigma_{n}\right\}$. In this case, we will simply write $\sigma_{i}=\operatorname{dev}\left(\mathfrak{c}_{i}\right)$ for the mapping between a (possibly ungrounded) constraint in the system and the corresponding derivation in the solution.

Theorem 6 (Soundness). Let $\Pi$ be a symbolic derivation of a ground sequent $\mathcal{G} \| \Gamma \vdash \Delta$. If $\mathcal{C}(\Pi)$ is solvable, then $\mathcal{G} \| \Gamma \vdash \Delta$ is derivable in $L S_{B B I}^{s f}$.

The proof is done by induction on the height of symbolic derivations. The basic idea of the proof is that one progressively "ground" a symbolic derivation, starting from the root of the derivation. At each inductive step we show that grounding the premises corresponds to restricting the constraint system induced by the symbolic derivation. The detailed proof can be found in Appendix A.13.

To prove the completeness of $F V L S_{B B I}$, we show that for every cut-free derivation $\Pi$ of a (ground) sequent in $L S_{B B I}^{s f}$, there is a symbolic derivation $\Pi^{\prime}$ of the same sequent such that $\mathcal{C}\left(\Pi^{\prime}\right)$ is solvable. It is quite obvious that $\Pi^{\prime}$ should have exactly the same rule applications as $\Pi$; the only difference is that some relational atoms are omitted in the derivation, but instead are accumulated in the constraint system. Additionally, some (new) labels are replaced with free variables. This is formalised in the following definition.

Definition 10. Given a sequent in a $L S_{B B I}^{s f}$ derivation, let $\mathcal{G}$ be the set of its relational atoms, we define $\mathcal{G}_{E}$ as the subset of $\mathcal{G}$ that contains those ternary relational atoms created by $* L,-* R$, and $\top^{*} L$. We define $\mathcal{G}_{S}=\mathcal{G} \backslash \mathcal{G}_{E}$. We refer to $\mathcal{G}_{E}$ as the essential subset of $\mathcal{G}$, and $\mathcal{G}_{S}$ as the supplementary subset of $\mathcal{G}$.

For a list $L$, we denote by $\operatorname{head}(L)$ the first element in the list $L$ and $\operatorname{tail}(L)$ the list of $L$ without the first element, and $\operatorname{end}(L)$ the last element in $L$. We denote by $L_{1} @ L_{2}$ the concatenation of two lists $L_{1}$ and $L_{2}$, and $\operatorname{pre}(x)$ the predecessor of $x$ in a list $L$, and $\operatorname{suc}(x)$ the successor of $x$ in $L$.

Given a well-formed constraint system $(\mathcal{C}, \preceq)$, we can define a partial order $\preceq^{v}$ on free variables of $\mathcal{C}$ as follows: $\mathbf{x} \preceq^{v} \mathbf{y}$ iff $\mathfrak{c}(\mathbf{x}) \preceq \mathfrak{c}(\mathbf{y})$. That is, free variables are ordered according to their originations. The relations $\prec^{v}$ and $\lessdot^{v}$ are defined analogously to $\prec$ and $\lessdot$, i.e., as the non-reflexive subset of $\preceq^{v}$ and the successor relation.

Definition 11 (A thread of variables). Let $\mathbb{C}=(\mathcal{C}, \preceq)$ be a well-formed constraint system, and let $X$ be a list of free variables $\mathbf{x}_{1}, \ldots, \mathbf{x}_{n}$, where $n \geq 0$. Let $\preceq^{v}$ be the partial order on variables, derived from $\preceq$. We say $X$ is a thread of free variables of $\mathbb{C}$ (or simply a thread of $\mathbb{C}$ ) iff it satisfies the following conditions:

1. $\forall \mathbf{x} \in X, \mathbf{x} \in f v(\mathcal{C})$

2. For every $i \in\{1, \ldots, n-1\}, \mathbf{x}_{i} \lessdot^{v} \mathbf{x}_{i+1}$.

3. If $n \geq 1$, then $\mathbf{x}_{1}$ is a minimum element and $\mathbf{x}_{n}$ is a maximum element of $\preceq^{v}$.

4. If $n \geq 1$, then $\mathfrak{c}\left(\mathbf{x}_{1}\right)$ is a minimum constraint in $\mathcal{C}$.

A thread is effectively those variables that are generated along a certain branch in a $F V L S_{B B I}$ symbolic derivation. It is not hard to verify that in a valid symbolic derivation in $F V L S_{B B I}$ of a ground sequent, the set of free variables in any symbolic sequent in the derivation can be linearly ordered as a thread.

Definition 12. Let $\mathbb{C}=\left(\mathcal{C}_{1}, \preceq_{1}\right)$ be a well-formed constraint system, let $X$ be a thread of $\mathbb{C}_{1}$ and let $\mathbb{C}_{2}=$ $\left(\mathcal{C}_{2}, \preceq_{2}\right)$ be a constraint system (but not neccessarily well-formed) such that $X$ consists of free variables in $f v\left(\mathcal{C}_{1}\right) \cap f v\left(\mathcal{C}_{2}\right)$. Furthermore, assume that every variable $\mathbf{x}$ in $\mathcal{C}^{\prime}$, except for those in $X$, satisfies the variable origination property, i.e., $\mathbf{x}$ originates from a constraint in $\mathcal{C}^{\prime}$. The composition of $\mathbb{C}_{1}$ and $\mathbb{C}_{2}$ along the thread $X$, written $\mathbb{C}_{1} \circ^{X} \mathbb{C}_{2}$, is the constraint system $(\mathcal{C}, \preceq)$ such that:

$-\mathcal{C}=\mathcal{C}_{1} \cup \mathcal{C}_{2} ;$ and

- For $\mathfrak{c}_{1}, \mathfrak{c}_{2} \in \mathcal{C}, \mathfrak{c}_{1} \preceq \mathfrak{c}_{2}$ iff either one of the following holds:

- $\mathfrak{c}_{1} \preceq_{1} \mathfrak{c}_{2}$, 
- $\mathfrak{c}_{1} \preceq_{2} \mathfrak{c}_{2}$, or

- $X$ is non-empty, $\mathbf{y}=\operatorname{end}(X), \mathfrak{c}_{1}=\mathfrak{c}(\mathbf{y})$ and $\mathfrak{c}_{2} \in \mathcal{C}_{2}$.

This definition basically says that the composition of $\mathbb{C}_{1}$ and $\mathbb{C}_{2}$ along $X$ is obtained by simply ordering the constraints so that all constraints $\mathcal{C}_{2}$ are greater than $\mathfrak{c}(\mathbf{y})$, where $\mathbf{y}$ is the last variable in $X$. If $X$ is empty, then $\mathcal{C}_{1}$ and $\mathcal{C}_{2}$ are independent, and $\prec$ is simply the union of $\prec_{1}$ and $\prec_{2}$.

Lemma 9. Let $(\mathcal{C}, \preceq)$ be as defined in Definition 12 . Then $(\mathcal{C}, \preceq)$ is well-formed.

Proof. This follows straightforwardly from the definition.

Lemma 10. Let $\mathbb{C}=(\mathcal{C}, \preceq)$ be a well-formed constraint system and let $X$ be a thread of $\mathbb{C}$. Let $\Pi$ be a symbolic derivation such that the free variables in its end sequent are exactly those in $X$. Then $\mathbb{C} \circ X \mathbb{C}(\Pi)$ is well-formed.

Definition 13. Let $\mathbb{C}=(\mathcal{C}, \preceq)$ be a well-formed constraint system and let $S=(\theta,\{\vec{\sigma}\})$ be its solution. Let $X$ be a thread of $\mathbb{C}$. Define a set of relational atoms $\mathcal{S}^{*}(\mathbb{C}, S, X)$ inductively as follows:

- If $n=0$ then $\mathcal{S}^{*}(\mathbb{C}, S,[])=\emptyset$

- Suppose $n>0$. Let head $(X)=\mathbf{x}$. Then $\mathfrak{c}(\mathbf{x}) \in \mathcal{C}$ is a minimum constraint of $\mathbb{C}$, and there exists $\sigma_{\mathbf{x}} \in\{\vec{\sigma}\}$ such that $\left(\theta_{\mathbf{x}}, \sigma_{\mathbf{x}}\right)$ is a solution to $\mathfrak{c}(\mathbf{x})$, where $\theta_{\mathbf{x}}=\theta \uparrow f v(\mathfrak{c}(\mathbf{x}))$. In this case, $\mathcal{S}^{*}(\mathbb{C}, S, X)$ is defined as follows.

$$
\mathcal{S}^{*}(\mathbb{C}, S, X)=\mathcal{S}\left(\mathcal{G}(\mathfrak{c}(\mathbf{x})), \sigma_{\mathbf{x}}\right) \cup \mathcal{S}^{*}\left(\mathbb{C} \uparrow\left(\mathfrak{c}(\mathbf{x}), \theta_{\mathbf{x}}, \sigma_{\mathbf{x}}\right), S^{\prime}, \operatorname{tail}(X)\right)
$$

where $S^{\prime}=\left(\theta \backslash \theta_{\mathbf{x}},\{\vec{\sigma}\} \backslash\left\{\sigma_{\mathbf{x}}\right\}\right)$.

Notice that by the definition of restriction to a constraint system, every time a minimum constraint $\mathfrak{c}_{\mathbf{x}}$ is eliminated in the second clause in the above definition, $\mathcal{S}\left(\mathcal{G}\left(\mathfrak{c}_{\mathbf{x}}\right), \sigma_{\mathbf{x}}\right)$ is also added to the left hand side of every successor constraints of $\mathfrak{c}_{\mathbf{x}}$ in $\mathcal{C}$. Therefore it is straightforward that the following proposition holds.

Proposition 1. Let $\mathbb{C}=(\mathcal{C}, \preceq)$ be a well-formed constraint system. Let $\mathcal{G}=\mathcal{S}^{*}(\mathbb{C}, S, X)$, for some thread $X$ of $\mathbb{C}$, let $\mathbf{x}_{e}=\operatorname{end}(X)$ and let $S=(\theta,\{\vec{\sigma}\})$ be a solution to $\mathbb{C}$. Let $\mathfrak{c}=\mathcal{G}_{\mathfrak{c}} \vdash_{R}^{?} C_{\mathfrak{c}}$ be a constraint not in $\mathcal{C}$, such that $\mathcal{G}_{\mathfrak{c}}$ only contains free variables that occur in $\mathbb{C}$. Let $\mathbf{x}$ be a new variable occurring only on the right hand side of $\mathfrak{c}$. Let $\mathbb{C}^{\prime}=\left(\mathcal{C}^{\prime}, \preceq^{\prime}\right)$ be the following constraint system:

$-\mathcal{C}^{\prime}=\mathcal{C} \cup\{\mathfrak{c}\}$

- $\preceq^{\prime}$ is the smallest extension of $\preceq$ such that $\mathfrak{c}\left(\mathbf{x}_{e}\right) \lessdot \mathfrak{c}$.

Let $\left(\theta_{x}, \sigma_{x}\right)$ be the solution to $\mathfrak{c}^{\prime}=\mathcal{G} \cup \mathcal{G}_{\mathfrak{c}} \theta \vdash{ }_{R}^{?} C_{\mathfrak{c}} \theta, S^{\prime}=\left(\theta \cup \theta_{x},\left\{\vec{\sigma}, \sigma_{x}\right\}\right)$, and $X^{\prime}=X @[\mathbf{x}]$. Then $\mathcal{S}^{*}\left(\mathbb{C}^{\prime}, S^{\prime}, X^{\prime}\right)=\mathcal{S}\left(\mathcal{G} \cup \mathcal{G}_{\mathfrak{c}} \theta, \sigma_{x}\right)$.

Theorem 7. Let $\Pi$ be a derivation of a sequent in $L S_{B B I}^{s f}$. Then there exists a symbolic derivation $\Pi^{\prime}$ of the same sequent such that $\mathcal{C}\left(\Pi^{\prime}\right)$ is solvable.

The heart of the proof for this theorem is that we can recover the supplementary subset for each sequent from the constraint system using $\mathcal{S}^{*}$. Since the constraint system accumulates the relational atoms in the derivation, this is not a surprising result. The proof is given in more details in Appendix A.14.

\section{A heuristics for proof search}

Suppose we want to prove $((a * b) * c) \rightarrow(a *(b * c))$. Using $F V L S_{B B I}$, we build a symbolic derivation as in Figure 9 (right associativity for connectives is assumed). The following constraints are generated from this derivation:

$\begin{array}{lll}(a 1, a 2 \triangleright a 0) ;(a 3, a 4 \triangleright a 1) & \vdash_{R} & (a 3=\mathbf{x} 5) \\ (a 1, a 2 \triangleright a 0) ;(a 3, a 4 \triangleright a 1) & \vdash_{R} & (a 4=\mathbf{x} 7) \\ (a 1, a 2 \triangleright a 0) ;(a 3, a 4 \triangleright a 1) & \vdash_{R} & (a 2=\mathbf{x} 8) \\ (a 1, a 2 \triangleright a 0) ;(a 3, a 4 \triangleright a 1) & \vdash_{R} & (\mathbf{x} 7, \mathbf{x} 8 \triangleright \mathbf{x} 6) \\ (a 1, a 2 \triangleright a 0) ;(a 3, a 4 \triangleright a 1) & \vdash_{R} & (\mathbf{x} 5, \mathbf{x} 6 \triangleright a 0)\end{array}$




$$
\begin{aligned}
& \text { Let } \Gamma_{1}:=\{a 2: c ; a 3: a\} \text { and } \Gamma_{2}:=\{a 3: a ; a 4: b\} \text { in }
\end{aligned}
$$

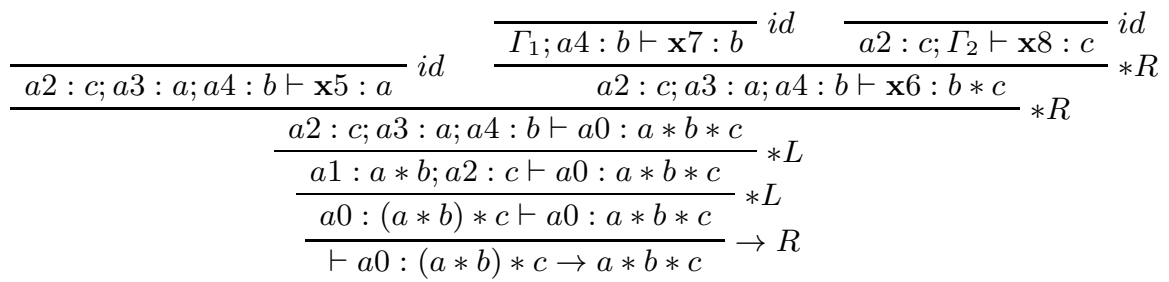

Fig. 9. A symbolic derivation for $((a * b) * c) \rightarrow(a *(b * c))$.

Since the first three constraints are required by the $i d$ rule, we must accept them. Thus we are only left with the last two constraints with free variables $\mathbf{x} 5, \mathbf{x} 7, \mathbf{x} 8$ assigned. In the following, we shall write $(a 1, a 2 \triangleright a 0) ;(a 3, a 4 \triangleright a 1)$ as $\mathcal{G}$, and $(a 3, \mathbf{x} 6 \triangleright a 0) ;(a 2, a 4 \triangleright \mathbf{x} 6)$ as $C$. Now $\mathbf{x} 6$ is the only remaining free variable. We can apply the rule $A$ (upward) on the left hand side $\mathcal{G}$ to obtain $(a 3, w \triangleright a 0)$; $(a 2, a 4 \triangleright w)$, where $w$ is a new label. Then apply the rule $E$ (upward) to obtain ( $a 4, a 2 \triangleright w)$. The two constraints can be solved by assigning $w$ to $\mathbf{x} 6$.

But there is a simpler way to see that such a $w$ must exist: the two ternary relational atoms in $\mathcal{G}$ manifest that $a 0$ can be split into $a 2, a 3, a 4$. This is exactly what $C$ says. For any variant of $\mathcal{G}$ that describes the same splitting of $a 0$ as $C$, the "internal" node $\mathbf{x} 6$ can always be assigned to either an existing label or a label generated by the associativity rule. In the example, $\mathbf{x} 6$ cannot be matched to any existing label, so we can assign $x 6$ to be a fresh label globally, and add $C$ to the l.h.s. of the successor constraints in the partial order $\prec$. Similarly for any variant of $C$ with the same splitting of $a 0$.

We can extend this method to a chain of multiple relational atoms which forms a labelled binary tree. We define a labelled binary tree as a binary tree where each node is associated with a label. Each node in a labelled binary tree has a left child and a right child. The minimum labelled binary tree has a root and two leaves, which corresponds to a single relational atom. We define the following function inductively from a labelled binary tree to a set of relational atoms.

Definition 14. Let $t r$ be a labelled binary tree, the set of relational atoms w.r.t. tr, written as Rel(tr), is defined as follows.

- (Base case): tr only contains a root node labelled with $r$ and two leaves labelled with a, $b$ respectively. Then $\operatorname{Rel}(t r)=\{(a, b \triangleright r)\}$

- (Inductive case): tr contains a root node labelled with $r$ and its left and right children labelled with a and $b$ respectively. Then $\operatorname{Rel}(t r)=\operatorname{Rel}\left(t r_{a}\right) \cup \operatorname{Rel}\left(t_{b}\right) \cup\{(a, b \triangleright r)\}$, where $\operatorname{tr}_{a}$ and $\operatorname{tr}_{b}$ are the subtrees rooted at, respectively, the left child and the right child of the root node of $t r$.

The width of a labelled binary tree is defined as the number of leaves in the tree. A labelled binary tree is a variant of another labelled binary tree if either they are exactly the same, or they differ only in the labels of the internal nodes.

We say that a set $R$ of relational atoms forms a labelled binary tree $t r$ when $R=\operatorname{Rel}(t r)$. In this case, the leaves in $t r$ are actually a "splitting" of the root node. Commutativity and associativity guarantee that we can split a node arbitrarily, as long as the leaves in the tree are the same. Moreover, since all internal nodes are free variables, we can assign them to either existing labels or fresh labels (created by $A, A_{C}$ ) without having clash with existing relational atoms. This idea is formalised in the following lemma, and is proved in Appendix A.15.

Lemma 11. Given constraints $\mathfrak{c}_{1} \lessdot \cdots \lessdot \mathfrak{c}_{n}$ with $\mathcal{G}=\mathcal{G}\left(\mathfrak{c}_{1}\right)=\cdots=\mathcal{G}\left(\mathfrak{c}_{n}\right)$ and the r.h.s. of these constraints gives the set $R$ of relational atoms. If the following hold:

1. $R=\operatorname{Rel}(t r)$, for some labelled binary tree $t r$ where every internal node label is a free variable $\mathbf{x}$ which only occurs once in $t r$, and $\mathfrak{c}_{1} \preceq \mathfrak{c}(\mathbf{x})$.

2. The other node labels in tr are non- $\epsilon$ labels.

3. There exist $\mathcal{G}^{\prime} \subseteq \mathcal{G}$ and $t r^{\prime}$ such that $\mathcal{G}^{\prime}=\operatorname{Rel}\left(t r^{\prime}\right)$ and $t r^{\prime}$ has the same root and leaves as $t r$.

Then $\mathfrak{c}_{1}, \cdots, \mathfrak{c}_{n}$ are solvable. 


\begin{tabular}{|l|l|l|l|}
\hline Formula & $\begin{array}{l}\text { BBeye } \\
\text { (opt) }\end{array}$ & $\begin{array}{l}\text { Naive } \\
\text { (Vamp })\end{array}$ & $\begin{array}{l}F V L S_{B B I} \\
\text { Heuristic }\end{array}$ \\
\hline$(a-* b) \wedge\left(\top *\left(\top^{*} \wedge a\right)\right) \rightarrow b$ & $\mathrm{~d}(2) 0$ & 0.003 & 0.001 \\
$\left(\top^{*}-* \neg\left(\neg a * \top^{*}\right)\right) \rightarrow a$ & $\mathrm{~d}(2) 0$ & 0.003 & 0.000 \\
$\neg((a-* \neg(a * b)) \wedge((\neg a-* \neg b) \wedge b))$ & $\mathrm{d}(2) 0$ & 0.004 & 0.001 \\
$\top^{*} \rightarrow((a-*(b-* c))-*((a * b)-* c))$ & $\mathrm{d}(2) 0.015$ & 0.017 & 0.001 \\
$\top^{*} \rightarrow((a *(b * c))-*((a * b) * c))$ & $\mathrm{d}(2) 0.036$ & 0.006 & 0.000 \\
$\top^{*} \rightarrow((a *((b-* e) * c))-*((a *(b-* e)) * c))$ & $\mathrm{d}(2) 0.07$ & 0.019 & 0.001 \\
$\neg((a-* \neg(\neg(d-* \neg(a *(c * b))) * a)) \wedge c *(d \wedge(a * b)))$ & $\mathrm{d}(2) 0.036$ & 0.037 & 0.001 \\
$\neg((c *(d * e)) \wedge B)$ where & $\mathrm{d}(2) 0.016$ & 0.075 & 0.039 \\
$B:=((a-* \neg(\neg(b-* \neg(d *(e * c))) * a)) *(b \wedge(a * \top)))$ & & & \\
$\neg(C *(d \wedge(a *(b * e))))$ where & $\mathrm{d}(3) 96.639$ & 0.089 & 0.038 \\
$\mathrm{C}:=((a-* \neg(\neg(d-* \neg((c * e) *(b * a))) * a)) \wedge c)$ & $\mathrm{d}(2) 0.009$ & 0.048 & 0.001 \\
$(a *(b *(c * d))) \rightarrow(d *(c *(b * a)))$ & $\mathrm{d}(3) 0.03$ & 0.07 & 0.001 \\
$(a *(b *(c * d))) \rightarrow(d *(b *(c * a)))$ & $\mathrm{d}(3) 1.625$ & 1.912 & 0.001 \\
$(a *(b *(c *(d * e)))) \rightarrow(e *(d *(a *(b * c))))$ & $\mathrm{d}(4) 20.829$ & 0.333 & 0.001 \\
$(a *(b *(c *(d * e)))) \rightarrow(e *(b *(a *(c * d))))$ & $\mathrm{d}(3) 6.258$ & 0.152 & 0.007 \\
\hline$T^{*} \rightarrow(a *((b-* e) *(c * d))-*((a * d) *(c *(b-* e))))$ & \\
\hline
\end{tabular}

Table 1. Initial experimental results.

\section{Experiment}

We used a Dell Optiplex 790 desktop with Intel CORE i7 $2600 @ 3.4 \mathrm{GHz}$ CPU and 8GB memory as the platform, and tested the following provers on the formulae from Park et al. [15]. (1) BBeye: the OCaml prover from Park et al. based upon nested sequents [15]; (2) Naive (Vamp): translates a BBI formula into a firstorder formula using the standard translation, then uses Vampire 2.6 6 6 to solve it; (3) FVLS $B B I$ Heuristic: backward proof search in $F V L S_{B B I}$, using the heuristic-based method to solve the set of constraints.

The results are shown in Table 1. The BBeye (opt) column shows the results from Park et al's prover where the $d()$ indicates the depth of proof search. The other two columns are for the two methods stated above. We see that naive translation is comparable with BBeye in most cases, but the latter is not stable. When the tested formulae involves more interaction between structural rules, BBeye runs significantly slower. The heuristic method outperforms all other methods in the tested cases.

Nonetheless, our prover is slower than BBeye for formulae which contain many occurrences of the same atomic formulae, giving (id) instances such as:

$$
\Gamma ; w_{1}: P ; w_{2}: P ; \cdots ; w_{n}: P \vdash \mathbf{x}: P ; \Delta
$$

We have to choose some $w_{i}$ to match with $\mathbf{x}$ without knowing which choice satisfies other constraints. In the worst case, we have to try each using backtracking. Multiple branches of this form lead to a combinatorial explosion. Determinising the concrete labels (worlds) for formulae in proof search in $L S_{B B I}$ or BBeye [15] avoids this problem. Further work is needed to solve this in $F V L S_{B B I}$.

Even though we do not claim the completeness of our heuristics method, it appears to be a fast way to solve certain problems. Completeness can be restored by fully implementing $L S_{B B I}$ or $F V L S_{B B I}$. The derivations in $L S_{B B I}$ are generally shorter than those in the Display Calculus or Nested Sequent Calculus for BBI. The reader can verify that most of formulae in Table 1 can even be proved by hand in a reasonable time using our labelled system. The optimisations of the implementation, however, is out of the scope of this paper.

\section{Conclusion and Future Work}

Our main contribution is a labelled sequent calculus for $B B I_{N D}$ that is sound, complete, and enjoys cutelimination. There are no explicit contraction rules in $L S_{B B I}$ and all structural rules can be restricted so that proof search is entirely driven by logical rules. We further propose a free variable system to restrict the proof search space so that some applications of $* R,-* L$ rules can be guided by zero-premise rules. Although we can structure proof search to be more manageable compared to the unrestricted (labelled or display) 


$$
\begin{array}{cc}
\frac{(a, b \triangleright c) ; \Gamma[c / d] \vdash \Delta[c / d]}{(a, b \triangleright c) ;(a, b \triangleright d) ; \Gamma \vdash \Delta} P & \frac{(a, b \triangleright c) ; \Gamma \vdash \Delta}{\Gamma \vdash \Delta}{ }_{T} \\
\frac{(\epsilon, \epsilon \triangleright \epsilon) ; \Gamma[\epsilon / a][\epsilon / b] \vdash \Delta[\epsilon / a][\epsilon / b]}{(a, b \triangleright \epsilon) ; \Gamma \vdash \Delta}{ }_{I U} & \frac{(a, b \triangleright c) ; \Gamma[b / d] \vdash \Delta[b / d]}{(a, b \triangleright c) ;(a, d \triangleright c) ; \Gamma \vdash \Delta} C
\end{array}
$$

In $T, a, b$ do occur in the conclusion but $c$ does not

In all substitutions $[y / x], x \neq \epsilon$

Fig. 10. Some auxiliary structural rules.

calculus, the undecidability of BBI implies that there is no terminating proof search strategy for a sound and complete system. The essence of proof search now resides in guessing which relational atom to use in the $* R$ and $-* L$ rules and whether they need to be applied more than once to a formula. Nevertheless, our initial experimental results already raise the hope that a more efficient proof search strategy can be developed based on our calculus.

An immediate task is to find a complete and terminating (if possible) constraint solving strategy. A counter-model construction for $B B I_{P D}$ has been studied by Larchey-Wendling using labelled tableaux [7, the possibility to adapt his method to $B B I_{N D}$ using our calculus is also a future work.

Another interesting topic is to extend our calculus to handle some semantics other than the nondeterministic monoidal ones. Our design of the structural rules in $L S_{B B I}$ can be generalised as follows. If there is a semantic condition of the form $\left(w_{11}, w_{12} \triangleright w_{13}\right) \wedge \cdots \wedge\left(w_{i 1}, w_{i 2} \triangleright w_{i 3}\right) \Rightarrow\left(w_{11}^{\prime}, w_{12}^{\prime} \triangleright w_{13}^{\prime}\right) \wedge \cdots \wedge$ $\left(w_{j 1}^{\prime}, w_{j 2}^{\prime} \triangleright w_{j 3}^{\prime}\right) \wedge\left(x_{11}=x_{12}\right) \wedge \cdots \wedge\left(x_{k 1}=x_{k 2}\right)$, we create a rule:

$$
\frac{\left(w_{11}^{\prime}, w_{12}^{\prime} \triangleright w_{13}^{\prime}\right) ; \cdots ;\left(w_{j 1}^{\prime}, w_{j 2}^{\prime} \triangleright w_{j 3}^{\prime}\right) ;\left(w_{11}, w_{12} \triangleright w_{13}\right) ; \cdots ;\left(w_{i 1}, w_{i 2} \triangleright w_{i 3}\right) ; \Gamma \vdash \Delta}{\left(w_{11}, w_{12} \triangleright w_{13}\right) ; \cdots ;\left(w_{i 1}, w_{i 2} \triangleright w_{i 3}\right) ; \Gamma \vdash \Delta} r
$$

And apply substitutions $\left[x_{12} / x_{11}\right] \cdots\left[x_{k 2} / x_{k 1}\right]$ globally on the premise, where $\epsilon$ is not substituted. Many additional features can be added in this way. We summarise the following desirable ones: (1) PD-semantics: the composition of two elements is either the empty set or a singleton, i.e., $(a, b \triangleright c) \wedge(a, b \triangleright d) \Rightarrow(c=d)$; (2) TD-semantics: the composition of any two elements is always defined as a singleton, i.e., $\forall a, b, \exists c$ s.t. $(a, b \triangleright c)$; (3) indivisible unit: (cf. Section 1) $(a, b \triangleright \epsilon) \Rightarrow(a=\epsilon) \wedge(b=\epsilon)$; and (4) cancellative: if $w \circ w^{\prime}$ is defined and $w \circ w^{\prime}=w \circ w^{\prime \prime}$, then $w^{\prime}=w^{\prime \prime}$, i.e., $(a, b \triangleright c) \wedge(a, d \triangleright c) \Rightarrow(b=d)$. Note that (2) and (4) are in addition to (1). The above are formalised in rules $P, T, I U, C$ respectively in Figure 10,

The formula

$$
(F * F) \rightarrow F, \text { where } F=\neg\left(\top-* \neg \top^{*}\right),
$$

differentiates $B B I_{N D}$ and $B B I_{P D}$ [9] and is provable using $L S_{B B I}+P$. Using $L S_{B B I}+T$, we can prove $\left(\neg \top^{*}-* \perp\right) \rightarrow \top^{*}$, which is valid in $B B I_{T D}$ but not in $B B I_{P D}\left[9\right.$, and also $\left(\top^{*} \wedge((p * q)-* \perp)\right) \rightarrow$ $((p-* \perp) \vee(q-* \perp))$, which is valid in separation models iff the composition is total [4. These additional rules do not break cut-elimination.

Oddly, the formula $\neg\left(\top^{*} \wedge A \wedge\left(B * \neg\left(C-*\left(\top^{*} \rightarrow A\right)\right)\right)\right)$, which is valid in $B B I_{N D}$, is very hard to prove in the display calculus and Park et al.'s method. We ran this formula using Park et al.'s prover for a week on a CORE i7 2600 processor, without success. Very short proofs of this formula exist in $L S_{B B I}$ or Larchey-Wendling and Galmiche's labelled tableaux (this formula must also be valid in $B B I_{P D}$ ). We are currently investigating this phenomenon.

Furthermore, we point out that Park et al.'s following observations on $B B I_{N D}$ [15, page 2] are incorrect:

1. A node can have multiple parent nodes, but each parent node determines a unique sibling node. Hence no node can have two parent nodes with the same sibling node.

2. A node can have multiple child nodes, but each child node determines another unique child node. Hence we can divide all child nodes into groups of two sibling nodes.

Observation 1 is the claim that partial-determinism holds and Observation 2 is the claim that cancellativity holds, but neither of these properties hold for $B B I_{N D}$, which is what their nested sequent calculus is designed for. An earlier version of Park et al.'s technical report said that the two observations also imply each other, which, by Larchey-Wendling and Galmiche's work [11, is only correct about partial-determinism 
(Observation 1) implying cancellativity (Observation 2). The other direction, however, is so far not known, and this claim does not appear in the latest version of Park et al.'s paper [15]. Interestingly, Park et al.'s prover BBeye could not prove Formula 1 which is valid if partial-determinism (Observation 1) holds. We believe that their prover for $B B I_{N D}$ is actually behaving correctly since Observation 1 is false in $B B I_{N D}$, so their own prover does not support their Observation 1 above.

The proofs for the formulae in this section can be found in Appendix A.16.

\section{References}

1. James Brotherston. A unified display proof theory for bunched logic. ENTCS, 265:197-211, September 2010.

2. James Brotherston and Cristiano Calcagno. Classical BI: Its semantics and proof theory. LMCS, 6(3), 2010.

3. James Brotherston and Max Kanovich. Undecidability of propositional separation logic and its neighbours. In LICS, pages 130-139, 2010.

4. James Brotherston and Max Kanovich. Undecidability of propositional separation logic and its neighbours. submitted to the Journal of ACM, 2013.

5. Didier Galmiche and Dominique Larchey-Wendling. Expressivity properties of Boolean BI through relational models. In FSTTCS, pages 358-369, 2006.

6. Krystof Hoder and Andrei Voronkov. Comparing unification algorithms in first-order theorem proving. KI'09, pages 435-443. Springer-Verlag, 2009.

7. Dominique Larchey-Wendling. The formal strong completeness of partial monoidal Boolean BI. Journal of Logic and Computation, 2014.

8. Dominique Larchey-Wendling and Didier Galmiche. Exploring the relation between intuitionistic BI and Boolean BI: An unexpected embedding. MSCS, 19(3):435-500, 2009.

9. Dominique Larchey-Wendling and Didier Galmiche. The undecidability of Boolean BI through phase semantics. LICS, 0:140-149, 2010.

10. Dominique Larchey-Wendling and Didier Galmiche. Non-deterministic phase semantics and the undecidability of Boolean BI. ACM TOCL, 14(1), 2013.

11. Dominique Larchey-Wendling and Didier Galmiche. Looking at separation algebras with Boolean BI-eyes. Theoretical Computer Science, TCS 2014, 2014.

12. Sara Negri. Proof analysis in modal logic. JPL, 34(5-6):507-544, 2005.

13. Sara Negri and Jan von Plato. Structural Proof Theory. CUP, 2001.

14. Peter W. O'Hearn and David J. Pym. The logic of bunched implications. BSL, 5(2):215-244, 1999.

15. Jonghyun Park, Jeongbong Seo, and Sungwoo Park. A theorem prover for Boolean BI. POPL' 13, pages 219-232, New York, NY, USA, 2013. ACM.

16. David J. Pym. The Semantics and Proof Theory of the Logic of Bunched Implications. Applied Logic Series. Kluwer Academic Publishers, 2002.

17. John C. Reynolds. Separation logic: A logic for shared mutable data structures. LICS '02, pages 55-74. IEEE Computer Society, 2002.

18. Anne S. Troelstra and Helmut Schwichtenberg. Basic Proof Theory. CUP, 1996.

\section{A Appendix}

This section provides the details of the proofs in this paper.

\section{A.1 Soundness of $L S_{B B I}$}

Proof for Theorem 1

Proof. To prove the soundness of $L S_{B B I}$, we show that each rule preserves falsifiability upwards, as this is a more natural direction in terms of backward proof search. Therefore to prove that a rule is sound, we need to show that if the conclusion is falsifiable, then at least one of the premises is falsifiable (usually in the same choice of $v, \rho$, and $\mathcal{M})$. Most of the cases are easy, we show some samples here.

$i d$ Since there is no premise in this rule, we simply need to show that the conclusion is not falsifiable.

Suppose the sequent $\Gamma ; w: P \vdash w: P ; \Delta$ is falsifiable, then $\Gamma$ must be true and $\rho(w) \Vdash A$ and $\rho(w) \Vdash A$ and $\Delta$ must be false. However, $\rho(w) \Vdash A$ and $\rho(w) \Vdash A$ cannot hold at the same time for any $(\mathcal{M}, \triangleright, \epsilon)$, $v$ and $\rho$, so we have a contradiction, thus this sequent is not falsifiable. 
$\top^{*} L$ Assume $\Gamma ; w: \top^{*} \vdash \Delta$ is falsifiable, then $\Gamma$ is true and $\rho(w) \Vdash \top^{*}$ and $\Delta$ is false.

From the semantics of $T^{*}$ we know that $\rho(w) \Vdash T^{*}$ iff $\rho(w)=\epsilon$. Therefore by choosing the same $\rho, v$, and $\mathcal{M}$ for the premise, replacing every $w$ by $\epsilon$ in $\Gamma$ and $\Delta$ preserves their valuations, as we know that $\rho(\epsilon)=\epsilon$. That is, $\Gamma[\epsilon / w]$ must be true and $\Delta[\epsilon / w]$ must be false. So the premise is falsifiable.

$* L$ Assume the conclusion is falsifiable, so under some $v, \rho, \mathcal{M}$, we have that $\Gamma$ is true and $\rho(z) \Vdash A * B$ and $\Delta$ is false.

From the semantics of $A * B$, we know that $\exists a, b$ s.t. $a, b \triangleright \rho(z)$ and $a \Vdash A$ and $b \Vdash B$. So we can choose a mapping $\rho^{\prime}$ with $\rho^{\prime}=(x \mapsto a) \cup(y \mapsto b) \cup \rho$. Since $x$ and $y$ are fresh, they should not affect anything in $\rho$. Then, under $\rho^{\prime}$, the following hold: $(x, y \triangleright z)$ is true and $\Gamma$ is true and $\rho^{\prime}(x) \Vdash A$ and $\rho^{\prime}(y) \Vdash B$ and $\Delta$ is false. Thus the premise is falsifiable in $v, \rho^{\prime}$, and $\mathcal{M}$.

$* R$ Assume under some $v, \rho$, and $\mathcal{M},(x, y \triangleright z)$ is true and $\Gamma$ is true and $\rho(z) \forall A * B$ and $\Delta$ is false.

The semantics of $A * B$ yields the following:

$$
\begin{aligned}
\rho(z) \Downarrow A * B & \Leftrightarrow \neg(\exists a, b .(a, b \triangleright \rho(z) \text { and } a \Vdash A \text { and } b \Vdash B)) \\
& \Leftrightarrow \forall a, b .(a, b \triangleright \rho(z) \text { doesn't hold or } a \Downarrow A \text { or } b \Downarrow B)
\end{aligned}
$$

If we pick the same set of $v, \rho, \mathcal{M}$ for the premises, however, in both premises the relational atom $(x, y \triangleright z)$ already exists, which means $\rho(x), \rho(y) \triangleright \rho(z)$ holds. So the possibility is only that either $\rho(x) \forall A$ or $\rho(y) \Downarrow B$. Assume the former one holds, then the left premise is falsifiable, otherwise the right premise is falsifiable.

Rules for additive connectives are straightforward, the cases for $-*$ can be proved similarly as for $*$ above. Structural rules $E, A$ (and $A_{C}$ ), $E q_{1}$ (and $E q_{2}$ and $U$ ) can be proved by using the commutativity, associativity, and identity properties of the monoid structure respectively.

\section{A.2 Substitution for labels}

The proof for Lemma 1

Proof. By induction on $h t(\Pi)$.

(Base case) If $h t(\Pi)=0$, then the only applicable rules are $i d, \perp L, \top R$ and $\top^{*} R$. If the label $x \neq \epsilon$ being substituted is not on the principal formula, then the substitution does not affect the original derivation. Note that since we do not allow to substitute for the label $\epsilon$, the proof for $T^{*} R$ can only be this case. Otherwise we obtain the new derivation by simply replacing the label of the principal formula.

(Inductive case) If $h t(\Pi)>0$, then consider the last rule applied in the derivation. We consider three main cases.

1. Neither $x$ nor $y$ is the label of the principal formula.

(a) Suppose the last rule applied is $T^{*} L$, and $x \neq w$ and $y \neq w$, and $\Pi$ is the following derivation:

$$
\frac{\Gamma^{\prime}[\epsilon / w] \vdash \Delta[\epsilon / w]}{\Gamma^{\prime} ; w: \top^{*} \vdash \Delta} \top^{*} L
$$

By the induction hypothesis, there is a derivation $\Pi_{1}^{\prime}$ of $\Gamma^{\prime}[\epsilon / w][y / x] \vdash \Delta[\epsilon / w][y / x]$ with $h t\left(\Pi_{1}^{\prime}\right) \leq$ $h t\left(\Pi_{1}\right)$. Since $x$ and $y$ are different from $w$, this sequent is equal to $\Gamma^{\prime}[y / x][\epsilon / w] \vdash \Delta[y / x][\epsilon / w]$. Therefore $\Pi^{\prime}$ is constructed as follows.

Obviously $h t\left(\Pi^{\prime}\right) \leq h t(\Pi)$.

$$
\frac{\Gamma^{\prime}[y / x][\epsilon / w] \vdash \Delta[y / x][\epsilon / w]}{\Gamma^{\prime}[y / x] ; w: \top^{*} \vdash \Delta[y / x]} \top^{*} L
$$

(b) If the last rule applied is $E q_{1}$, we distinguish the following cases: $x$ is not $w$ or $w^{\prime} ; x=w ; x=w^{\prime}$.

i. $x \neq w$ and $x \neq w^{\prime}$. The original derivation is as follows.

$$
\frac{\Pi_{1}}{(\epsilon, w \triangleright w) ; \Gamma^{\prime}\left[w / w^{\prime}\right] \vdash \Delta\left[w / w^{\prime}\right]} \quad E q_{1}
$$


A. If $y \neq w$ and $y \neq w^{\prime}$, by the induction hypothesis, there is a derivation $\Pi_{1}^{\prime}$ of $(\epsilon, w \triangleright$ $w) ; \Gamma^{\prime}\left[w / w^{\prime}\right][y / x] \vdash \Delta\left[w / w^{\prime}\right][y / x]$ with $h t\left(\Pi_{1}^{\prime}\right) \leq h t\left(\Pi_{1}\right)$. Since $x, y, w, w^{\prime}$ are different labels, this sequent is equal to $(\epsilon, w \triangleright w) ; \Gamma^{\prime}[y / x]\left[w / w^{\prime}\right] \vdash \Delta[y / x]\left[w / w^{\prime}\right]$. Thus the derivation $\Pi^{\prime}$ is constructed as follows.

$$
\begin{gathered}
\Pi_{1}^{\prime} \\
\frac{(\epsilon, w \triangleright w) ; \Gamma^{\prime}[y / x]\left[w / w^{\prime}\right] \vdash \Delta[y / x]\left[w / w^{\prime}\right]}{\left(\epsilon, w^{\prime} \triangleright w\right) ; \Gamma^{\prime}[y / x] \vdash \Delta[y / x]} E q_{1}
\end{gathered}
$$

B. If $y=w$, this case is similar to Case 1.(b).i.A.

C. Suppose $y=w^{\prime}$. Then we need to derive $(\epsilon, y \triangleright w) ; \Gamma^{\prime}[y / x] \vdash \Delta[y / x]$. If $y \neq \epsilon$, we construct $\Pi^{\prime}$ by first applying $E q_{1}$ bottom-up:

$$
\frac{(\epsilon, w \triangleright w) ; \Gamma^{\prime}[y / x][w / y] \vdash \Delta[y / x][w / y]}{(\epsilon, y \triangleright w) ; \Gamma^{\prime}[y / x] \vdash \Delta[y / x]} E q_{1}
$$

Now the premise is equal to $(\epsilon, w \triangleright w) ; \Gamma^{\prime}[w / y][w / x] \vdash \Delta[w / y][w / x]$, and by the induction hypothesis, there is a derivation $\Pi_{1}^{\prime}$ of this sequent, with $h t\left(\Pi_{1}^{\prime}\right) \leq h t\left(\Pi_{1}\right)$.

If $y=\epsilon$, then we need to apply $E q_{2}$, instead of $E q_{1}$ :

$$
\frac{(\epsilon, \epsilon \triangleright \epsilon) ; \Gamma^{\prime}[\epsilon / x][\epsilon / w] \vdash \Delta[\epsilon / x][\epsilon / w]}{(\epsilon, \epsilon \triangleright w) ; \Gamma^{\prime}[\epsilon / x] \vdash \Delta[\epsilon / x]} E q_{2}
$$

Note that the sequent $(\epsilon, \epsilon \triangleright \epsilon) ; \Gamma^{\prime}[\epsilon / x][\epsilon / w] \vdash \Delta[\epsilon / x][\epsilon / w]$ is the same as

$$
(\epsilon, \epsilon \triangleright \epsilon) ; \Gamma^{\prime}\left[w / w^{\prime}\right][\epsilon / w][\epsilon / x] \vdash \Delta\left[w / w^{\prime}\right][\epsilon / w][\epsilon / x] .
$$

So the premise can be proved by two successive applications of the induction hypothesis to $\Pi_{1}$, one using substitution $[\epsilon / w]$ and the other using substitution $[\epsilon / x]$. Here we can apply the induction hypothesis twice to $\Pi_{1}$ because substitution does not increase the height of derivations.

ii. $x=w$ (so $w$ cannot be $\epsilon$ ).

A. If $y \neq w^{\prime}$, then $\Pi$ has the form:

$$
\begin{gathered}
\Pi_{1} \\
\frac{(\epsilon, x \triangleright x) ; \Gamma^{\prime}\left[x / w^{\prime}\right] \vdash \Delta\left[x / w^{\prime}\right]}{\left(\epsilon, w^{\prime} \triangleright x\right) ; \Gamma^{\prime} \vdash \Delta} E q_{1}
\end{gathered}
$$

By the induction hypothesis we have the folowing derivation:

$$
\Pi_{1}^{\prime}
$$

The end sequent is equal to the following:

$$
(\epsilon, y \triangleright y) ; \Gamma^{\prime}\left[x / w^{\prime}\right][y / x] \vdash \Delta\left[x / w^{\prime}\right][y / x]
$$

$$
(\epsilon, y \triangleright y) ; \Gamma^{\prime}[y / x]\left[y / w^{\prime}\right] \vdash \Delta[y / x]\left[y / w^{\prime}\right] .
$$

Then by using $E q_{1}$, we construct $\Pi^{\prime}$ as follows.

B. If $y=w^{\prime}$, then $\Pi$ has the form:

$$
\frac{\Pi_{1}^{\prime}}{(\epsilon, y \triangleright y) ; \Gamma^{\prime}[y / x]\left[y / w^{\prime}\right] \vdash \Delta[y / x]\left[y / w^{\prime}\right]} \quad E q_{1}
$$

$$
\frac{\Pi_{1}}{(\epsilon, x \triangleright x) ; \Gamma^{\prime}[x / y] \vdash \Delta[x / y]} \frac{}{(\epsilon, y \triangleright x) ; \Gamma^{\prime} \vdash \Delta} E q_{1}
$$

By the induction hypothesis, we have the following derivation:

$$
\Pi_{1}^{\prime}
$$

$$
(\epsilon, y \triangleright y) ; \Gamma^{\prime}[x / y][y / x] \vdash \Delta[x / y][y / x]
$$

Since in the end sequent, we replace every $y$ by $x$, and then change every $x$ back to $y$, the effect is the same as just keeping every $y$ unchanged and only replace every $x$ by $y$. Thus the end sequent is equal to:

$$
(\epsilon, y \triangleright y) ; \Gamma^{\prime}[y / x] \vdash \Delta[y / x]
$$

which is exactly what we need to derive. Therefore we let $\Pi^{\prime}=\Pi_{1}^{\prime}$. Notice that in this case $h t\left(\Pi^{\prime}\right)<h t(\Pi)$.

iii. $x=w^{\prime}$.

A. If $y \neq w$ and $y \neq \epsilon$, the original derivation is as follows. 


$$
\begin{gathered}
\Pi_{1} \\
\frac{(\epsilon, w \triangleright w) ; \Gamma^{\prime}[w / x] \vdash \Delta[w / x]}{(\epsilon, x \triangleright w) ; \Gamma^{\prime} \vdash \Delta} E q_{1}
\end{gathered}
$$

By the induction hypothesis (instead of replacing every $x$ by $y$, we now replace every $y$ by $w)$, we have the following derivation:

The end sequent is equal to:

$$
(\epsilon, w \triangleright w) ; \Gamma^{\prime}[w / x][w / y] \vdash \Delta[w / x][w / y]
$$

$$
(\epsilon, w \triangleright w) ; \Gamma^{\prime}[y / x][w / y] \vdash \Delta[y / x][w / y]
$$

Thus $\Pi^{\prime}$ is constructed as follows.

$$
\begin{gathered}
\Pi_{1}^{\prime} \\
\frac{(\epsilon, w \triangleright w) ; \Gamma^{\prime}[y / x][w / y] \vdash \Delta[y / x][w / y]}{(\epsilon, y \triangleright w) ; \Gamma^{\prime}[y / x] \vdash \Delta[y / x]} E q_{1}
\end{gathered}
$$

B. If $y=\epsilon$ and $w \neq \epsilon$, we need to derive the following sequent:

$$
(\epsilon, \epsilon \triangleright w) ; \Gamma^{\prime}[\epsilon / x] \vdash \Delta[\epsilon / x]
$$

By induction hypothesis, replacing every $w$ by $\epsilon$ in $\Pi_{1}$, then using the rule $E q_{2}$, we get the new derivation:

$$
\begin{gathered}
\Pi_{1}^{\prime} \\
\frac{(\epsilon, \epsilon \triangleright \epsilon) ; \Gamma^{\prime}[\epsilon / x][\epsilon / w] \vdash \Delta[\epsilon / x][\epsilon / w]}{(\epsilon, \epsilon \triangleright w) ; \Gamma^{\prime}[\epsilon / x] \vdash \Delta[\epsilon / x]} E q_{2}
\end{gathered}
$$

C. If $y=w$, then the premise of the last rule is exactly what we need to derive.

(c) If the last rule applied is $E q_{2}$, we consider three cases: $x \neq w$ and $y \neq w ; x=w$; and $y=w$. These are symmetric to the case where the last rule is $E q_{1}$, already discussed above.

2. $y$ is the label of the principal formula. Most of the cases follow similarly as above, except for $\top^{*} L$. In this case the original derivation is as follows.

$$
\begin{gathered}
\Pi_{1} \\
\frac{\Gamma^{\prime}[\epsilon / y] \vdash \Delta[\epsilon / y]}{\Gamma^{\prime} ; y: \top^{*} \vdash \Delta} \top^{*} L
\end{gathered}
$$

Our goal is to derive $\Gamma^{\prime}[y / x] ; y: \top^{*} \vdash \Delta[y / x]$. Applying $\top^{*} L$ as in backward proof search, we get

$$
\Gamma^{\prime}[y / x][\epsilon / y] \vdash \Delta[y / x][\epsilon / y]
$$

Note that this sequent is equal to $\Gamma^{\prime}[\epsilon / y][\epsilon / x] \vdash \Delta[\epsilon / y][\epsilon / x]$, and from induction hypothesis we know that there is a derivation of this sequent of height less than or equal to $h t(\Pi)$.

3. $x$ is the label of the principal formula.

(a) For the additive rules, since the labels stay the same in the premises and conclusions of the rules, even if the label of the principal formula is replaced by some other label, we can still apply the induction hypothesis on the premise, then use the rule to derive the conclusion.

For $\wedge L$,

$$
\begin{aligned}
& \Pi_{1} \\
& \Pi_{1}^{\prime} \\
& \frac{\Gamma^{\prime} ; x: A ; x: B \vdash \Delta}{\Gamma^{\prime} ; x: A \wedge B \vdash \Delta} \wedge L \quad \rightsquigarrow \quad \frac{\Gamma^{\prime}[y / x] ; y: A ; y: B \vdash \Delta[y / x]}{\Gamma^{\prime}[y / x] ; y: A \wedge B \vdash \Delta[y / x]} \wedge L
\end{aligned}
$$

For $\wedge R$,

$$
\text { For } \rightarrow L,
$$

$$
\begin{aligned}
& \Pi_{1} \quad \Pi_{2} \\
& \frac{\Gamma^{\prime} \vdash x: A ; \Delta \quad \Gamma^{\prime} \vdash x: B ; \Delta}{\Gamma^{\prime} \vdash x: A \wedge B ; \Delta} \wedge R \rightsquigarrow \\
& \Pi_{1}^{\prime} \quad \Pi_{2}^{\prime} \\
& \frac{\Gamma^{\prime}[y / x] \vdash y: A ; \Delta[y / x] \quad \Gamma^{\prime}[y / x] \vdash y: B ; \Delta[y / x]}{\Gamma^{\prime}[y / x] \vdash y: A \wedge B ; \Delta[y / x]} \wedge R
\end{aligned}
$$




$$
\begin{aligned}
& \Pi_{1} \quad \Pi_{2} \\
& \frac{\Gamma^{\prime} \vdash x: A ; \Delta \quad \Gamma^{\prime} ; x: B \vdash \Delta}{\Gamma^{\prime} ; x: A \rightarrow B \vdash \Delta} \rightarrow L^{\rightsquigarrow} \\
& \Pi_{1}^{\prime} \quad \Pi_{2}^{\prime} \\
& \frac{\Gamma^{\prime}[y / x] \vdash y: A ; \Delta[y / x] \quad \Gamma^{\prime}[y / x] ; y: B \vdash \Delta[y / x]}{\Gamma^{\prime}[y / x] ; y: A \rightarrow B \vdash \Delta[y / x]} \rightarrow L \\
& \text { For } \rightarrow R \text {, } \\
& \Pi_{1} \\
& \frac{\Gamma^{\prime} ; x: A \vdash x: B ; \Delta}{\Gamma^{\prime} \vdash x: A \rightarrow B ; \Delta} \rightarrow R \quad \rightsquigarrow \quad \frac{\Gamma^{\prime}[y / x] ; y: A \vdash y: B ; \Delta[y / x]}{\Gamma^{\prime}[y / x] \vdash y: A \rightarrow B ; \Delta[y / x]} \rightarrow R
\end{aligned}
$$

(b) For multiplicative rules that do not produce eigenvariables $\left(* R,-* L, \top^{*} L\right)$, we can proceed similarly as in the additive cases, except for the $T^{*} L$ rule. For the $T^{*} L$ rule, if the label $x$ of the principal formula is replaced by some (other) label $y$, i.e., $\Pi$ is

$$
\frac{\Pi_{1}}{\Gamma^{\prime}[\epsilon / x] \vdash \Delta[\epsilon / x]} \frac{\Gamma^{\prime} ; x: \top^{*} \vdash \Delta}{\Gamma^{*} L}
$$

then we then need a derivation of the sequent $\Gamma^{\prime}[y / x] ; y: \top^{*} \vdash \Delta[y / x]$. Using $\top^{*} L$ rule we have:

$$
\frac{\Gamma[y / x][\epsilon / y] \vdash \Delta[y / x][\epsilon / y]}{\Gamma[y / x] ; y: \top^{*} \vdash \Delta[y / x]} \top^{*} L
$$

Note that the premise now is equal to $\Gamma[\epsilon / x][\epsilon / y] \vdash \Delta[\epsilon / x][\epsilon / y]$, and can be proved using the induction hypothesis on $\Pi_{1}$.

If $y=\epsilon$, then $\Pi^{\prime}$ is obtained by applying Lemma 12 to $\Pi_{1}$.

(c) For the multiplicative rules that have eigenvariables $(* L$ and $-* R)$, if the label of the principal formula is replaced by a label other than the newly created labels in the rules, then we proceed similarly as in additive cases. If the label of the principal formula is replaced by one of the newly created labels, then we just need to create a different new label in the new relation.

For $* L$, we have the derivation:

$$
\begin{gathered}
\Pi_{1} \\
\frac{(y, z \triangleright x) ; \Gamma^{\prime} ; y: A ; z: B \vdash \Delta}{\Gamma^{\prime} ; x: A * B \vdash \Delta} * L
\end{gathered}
$$

If $x$ is substituted by $y$ (the case for substituting to $z$ is symmetric), then we need a derivation of $\Gamma^{\prime}[y / x] ; y: A * B \vdash \Delta[y / x]$. Note that since the $* L$ rule requires the relation $(y, z \triangleright x)$ to be fresh, so in the original derivation $y$ and $z$ cannot be in $\Gamma$ or $\Delta$. Therefore by induction hypothesis we must have a derivation $\Pi_{1}^{\prime}$ for

$$
\left(y^{\prime}, z^{\prime} \triangleright x\right) ; \Gamma^{\prime} ; y^{\prime}: A ; z^{\prime}: B \vdash \Delta,
$$

where $y^{\prime}$ and $z^{\prime}$ are new labels, such that $h t\left(\Pi_{1}^{\prime}\right) \leq h t\left(\Pi_{1}\right)$. Applying the induction hypothesis again to $\Pi_{1}^{\prime}$, we have a derivation $\Pi_{1}^{\prime \prime}\left(y^{\prime}, z^{\prime} \triangleright y\right) ; \Gamma^{\prime}[y / x] ; y^{\prime}: A ; z^{\prime}: B \vdash \Delta[y / x]$, with $h t\left(\Pi_{1}^{\prime \prime}\right) \leq h t\left(\Pi_{1}\right)$. Thus the derivation $\Pi^{\prime}$ is constructed as follows." $\Pi_{1}^{\prime \prime}$

The case for $-* R$ is similar. suppose $\Pi$ is:

$$
\frac{\left(y^{\prime}, z^{\prime} \triangleright y\right) ; \Gamma^{\prime}[y / x] ; y^{\prime}: A ; z^{\prime}: B \vdash \Delta[y / x]}{\Gamma^{\prime}[y / x] ; y: A * B \vdash \Delta[y / x]} * L
$$

\section{$\Pi_{1}$}

$$
\frac{(y, x \triangleright z) ; \Gamma ; y: A \vdash z: B ; \Delta^{\prime}}{\Gamma \vdash x: A-* B ; \Delta^{\prime}}-* R
$$

If $x$ is replaced by $y$, then we have the following derivation.

$$
\Pi_{1}^{9}
$$

$$
\frac{\left(y^{\prime}, y \triangleright z^{\prime}\right) ; \Gamma[y / x] ; y^{\prime}: A \vdash z^{\prime}: B ; \Delta^{\prime}[y / x]}{\Gamma[y / x] \vdash y: A-* B ; \Delta^{\prime}[y / x]}-* R
$$

If $x$ is replaced by $z$, then we have the following derivation.

$$
\Pi_{1}^{\prime}
$$

$$
\frac{\left(y^{\prime}, z \triangleright z^{\prime}\right) ; \Gamma[z / x] ; y^{\prime}: A \vdash z^{\prime}: B ; \Delta^{\prime}[z / x]}{\Gamma[z / x] \vdash z: A-* B ; \Delta^{\prime}[z / x]}-* R
$$




\section{A.3 Weakening admissibility of $L S_{B B I}$}

Lemma 12. For all structures $\Gamma, \Delta$, labelled formula $w: A$, and ternary relation $(x, y \triangleright z)$, if $\Gamma \vdash \Delta$ is derivable, then there exists a derivation of the same height for each of the following sequents:

$$
\Gamma ; w: A \vdash \Delta \quad \Gamma \vdash w: A ; \Delta \quad(x, y \triangleright z) ; \Gamma \vdash \Delta .
$$

Proof. By induction on $h t(\Pi)$. Since $i d, \perp L, \top R$, and $T^{*} R$ all have weakening built in, the base case trivially holds. For the inductive cases, the only nontrivial case is for $* L$ and $-* R$, where new labels have to be introduced. These labels can be systematically renamed to make sure that they do not clash with the labels in the weakened formula/relational atom.

This yields the proof for Lemma 2 in the paper. Furthermore, we can prove more useful lemmas based on the weakening property.

The next lemma shows that the assumption $\epsilon: T^{*}$ in the antecedent of a sequent is not used in any derivation, and since there is no rule that can be applied to it, so it can be removed without affecting provability.

Lemma 13. If $\Gamma ; \epsilon: \top^{*} \vdash \Delta$ is derivable, then $\Gamma \vdash \Delta$ is derivable with the same series of rule applications.

Proof. By a straightforward induction on the height of derivation $n$.

(Base case) If $n=0$, then $\Gamma ; \epsilon: \top^{*} \vdash \Delta$ must be the conclusion of one of $i d, \perp L, \top R, \top^{*} R$. Note that $\epsilon: T^{*}$ in the antecedent cannot be the principal formula of any of those rules, therefore those rules are applicable to $\Gamma \vdash \Delta$ as well.

(Inductive case) If $n>0$, consider the last rule in the derivation. It is obvious that $\epsilon: \top^{*}$ in the antecedent of a sequent cannot be the principal formula of any rules, therefore it has to appear in the premise(s) of the last rule. Thus we can apply the induction hypothesis on the premise(s) and then use the corresponding rule to derive $\Gamma \vdash \Delta$.

In general, if a formula is never principal in a derivation, it can obviously be omitted.

Lemma 14. If $w: A$ is not the principal formula of any rule application in the derivation of $\Gamma ; w: A \vdash \Delta$ $(\Gamma \vdash w: A ; \Delta$ resp.), then there is a derivation of $\Gamma \vdash \Delta$ with the same series of rule applications.

If we combine the above lemma and the admissibility of weakening, then we can replace a formula that is never used in a derivation by any structure.

Lemma 15. If $w: A$ is not the principal formula of any rule application (even though the label might be changed) in the derivation of $\Gamma ; w: A \vdash \Delta\left(\Gamma \vdash w: A ; \Delta\right.$ resp.), then there is a derivation of $\Gamma ; \Gamma^{\prime} \vdash \Delta$ $\left(\Gamma \vdash \Delta^{\prime} ; \Delta\right.$ resp.), and in the new derivation, the structure $\Gamma$ ( $\Delta$ resp.) is not altered except that certain labels in $\Gamma$ ( $\Delta$ resp.) are changed.

Proof. By induction on the height of derivation $n$.

(Base case) If $n=0$, since $w: A$ is not the principal formula, the substituted sequent is also the conlcusion of rules $i d, \perp L, \top R, T^{*} R$. This is the same as the base case of the proof for Lemma 13 ,

(Inductive case) If $n>0$, consider the last rule in the derivation. Since $w: A$ is not the principal formula, for all rules except $\top^{*} L$, the original derivation has $w: A$ in the premise(s) of the last rule, therefore we can apply the induction hypothesis on the premise(s) and then use the rule to get the desired derivation. We give an example here.

For $\wedge L$, suppose $w: A$ is in the antecedent, the original derivation is converted as follows.

$$
\begin{aligned}
& \Pi \quad \Pi^{\prime} \\
& \frac{\Gamma ; w: A ; x: B ; x: C \vdash \Delta}{\Gamma ; w: A ; x: B \wedge C \vdash \Delta} \wedge L \quad \rightsquigarrow \quad \frac{\Gamma ; \Gamma^{\prime} ; x: B ; x: C \vdash \Delta}{\Gamma ; \Gamma^{\prime} ; x: B \wedge C \vdash \Delta} \wedge L
\end{aligned}
$$

Other cases except $T^{*} L$ are similar.

If the last rule is $T^{*} L$, then we convert the derivation as follows. 


$$
\begin{array}{cc}
\Pi & \Pi^{\prime} \\
\frac{\Gamma[\epsilon / w] ; \epsilon: A \vdash \Delta[\epsilon / w]}{\Gamma ; w: A ; w: \top^{*} \vdash \Delta} \top^{*} L & \frac{\Gamma[\epsilon / w] ; \Gamma^{\prime}[\epsilon / w] \vdash \Delta[\epsilon / w]}{\Gamma ; \Gamma^{\prime} ; w: \top^{*} \vdash \Delta} \top^{*} L
\end{array}
$$

Note that we incorporate two steps here. First, by induction hypothesis, we have a derivation of $\Gamma[\epsilon / w] ; \Gamma^{\prime} \vdash$ $\Delta[\epsilon / w]$. Then by the Substitution Lemma, there is a derivation $\Pi^{\prime}$ of $\Gamma[\epsilon / w] ; \Gamma^{\prime}[\epsilon / w] \vdash \Delta[\epsilon / w]$, from which we can derive the final sequent.

Therefore the only change to $\Gamma^{\prime}$ in the new derivation is that some of its labels might be changed by the rules $T^{*} L, E q_{1}$, or $E q_{2}$.

Note 1. The admissibility of general weakening shows that if $\Gamma \vdash \Delta$ is derivable, then $\Gamma ; \Gamma^{\prime} \vdash \Delta ; \Delta^{\prime}$ is derivable. A stronger argument here is that in the derivation of the latter sequent, $\Gamma^{\prime}$ and $\Delta^{\prime}$ are never changed except that some labels might be changed. This is similar as in Lemma 15.

\section{A.4 Invertibility of rules in $L S_{B B I}$}

Proof for Lemma 3

Proof. As the additive rules in $L S_{B B I}$ are exactly the same as those in Negri's labelled system for Modal logic or $G 3 c$ (cf. [13]), the proof for them is similar. The main difference is that the rest of our rules are of different forms. However, as most of our rules do not modify the side structures, simply by applying the induction hypothesis and then using the corresponding rule, we get the new derivation. The cases where the last rule applied is $T^{*} L, E q_{1}$, or $E q_{2}$ follow essentially the same, except a global substitution needs to be considered, but that is of no harm.

Rules $E, A, U, A_{C}, * R$ and $-* L$ are trivially invertible as the conclusion is a subset of the premise, and weakening is height-preserving admissible.

To prove the cases for $* L$ and $-* R$, we do inductions on the height $n$ of the derivation. In each case below, it is obvious that each premise is always cut-free derivable with less or same height as the conclusion.

The case for $* L$ is as follows.

(Base case) If $n=0$, then the conclusion of $* L$ is one of the conlucsions of $i d, \perp L, \top R, \top^{*} R$, notice that the identity rule is restricted to propositions, therefore the premise of $* L$ is also the conclusions of the corresponding axiom rule.

(Inductive case) If $n>0$, and the last rule applied is not $* L$ or $-* R$, then no fresh labels are involved, so we can safely apply the induction hypothesis on the premise of the last rule and then use the rule to get the derivation. If the last rule is $* L$ or $-* R$, but the principal formula is in $\Gamma$ or $\Delta$, we proceed similarly, and use the Substitution Lemma to ensure that the eigenvariables are new. If the principal formula is $z: A * B$, then the premise of the last rule yields the desired conclusion.

The case for $-* R$ follows similarly.

For $T^{*} L$, again, we do an induction on the height $n$ of the derivation.

(Base case) If $n=0$, then $\Gamma ; x: \top^{*} \vdash \Delta$ is the conclusion of one of $i d, \perp L, \top R, \top^{*} R$, and $x: \top^{*}$ cannot be the principal formula. Note that in the first three cases the principal formulae can be labelled with anything. Since, in the sequent $\Gamma[\epsilon / x] \vdash \Delta[\epsilon / x]$, the label $x$ is uniformly replaced by $\epsilon$, this sequent can be the conclusion of the corresponding rule as well. For $T^{*} R$, since $T^{*}$ on the right hand side can only be labelled with $\epsilon$, so replacing $x$ to $\epsilon$ does not change its label. Thus this case is not broken either.

(Inductive case) If $n>0$, consider the last rule applied in the derivation.

1. If the principal formula or relation does not involve the label $x$, then we can apply the induction hypothesis directly on the premise of the last rule, then use the last rule to get the derivation.

2. Otherwise, if the principal formula or relation has label $x$, and the last rule is not $\top^{*} L$, we proceed similarly, except replacing the label in the principal relation or formula. The detail is exemplified using $* L$.

For $* L$, we have the following derivation:

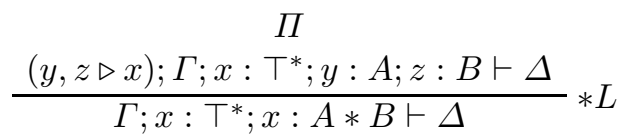


The condition of the rule $* L$ guarantees that $y$ and $z$ cannot be in $\Gamma$ and $\Delta$, so we do not have to worry if they are identical to $x$. By applying the induction hypothesis and then using the rule, we get the following derivation:

$$
\begin{gathered}
\Pi^{\prime} \\
\frac{(y, z \triangleright \epsilon) ; \Gamma[\epsilon / x] ; y: A ; z: B \vdash \Delta[\epsilon / x]}{\Gamma[\epsilon / x] ; \epsilon: A * B \vdash \Delta[\epsilon / x]} * L
\end{gathered}
$$

Another way to do this is by using the Substitution Lemma, replacing $x$ by $\epsilon$, we get a derivation to the premise that has a redundant $\epsilon: T^{*}$, since we know that this labelled formula on the left hand side does not contribute to the derivation, we can safely derive the sequent without it using the same inference, cf. Lemma 14 .

The case where the last rule is $-* R$ is similar.

If the last rule is $E q_{1}$, we consider the following cases:

(a) The label of $\top^{*}$ is not in the principal relation (i.e., $x \neq w$ and $x \neq w^{\prime}$ ). The original derivation is as follows.

$$
\frac{\Pi}{\frac{\Pi}{(\epsilon, w \triangleright w) ; \Gamma\left[w / w^{\prime}\right] ; x: \top^{*} \vdash \Delta\left[w / w^{\prime}\right]}}=q_{1}
$$

By the induction hypothesis, we have the following derivation:

$$
\begin{gathered}
\Pi^{\prime} \\
(\epsilon, w \triangleright w) ; \Gamma\left[w / w^{\prime}\right][\epsilon / x] \vdash \Delta\left[w / w^{\prime}\right][\epsilon / x]
\end{gathered}
$$

Note that since $x, w, w^{\prime}$ are all different, the end sequent is equal to the following:

$$
(\epsilon, w \triangleright w) ; \Gamma[\epsilon / x]\left[w / w^{\prime}\right] \vdash \Delta[\epsilon / x]\left[w / w^{\prime}\right]
$$

from which we can use the rule $E q_{1}$ and derive $\left(\epsilon, w^{\prime} \triangleright w\right) ; \Gamma[\epsilon / x] \vdash \Delta[\epsilon / x]$.

(b) $x=w$. The original derivation is as follows.

$$
\frac{\Pi}{(\epsilon, x \triangleright x) ; \Gamma\left[x / w^{\prime}\right] ; x: \top^{*} \vdash \Delta\left[x / w^{\prime}\right]}=q_{1}
$$

By the substitution lemma, replacing every $x$ by $\epsilon$ in the premise of the last rule, we get the following derivation:

The end sequent is equal to:

$$
\begin{gathered}
\Pi^{\prime} \\
(\epsilon, \epsilon \triangleright \epsilon) ; \Gamma\left[x / w^{\prime}\right][\epsilon / x] ; \epsilon: \top^{*} \vdash \Delta\left[x / w^{\prime}\right][\epsilon / x]
\end{gathered}
$$

$$
(\epsilon, \epsilon \triangleright \epsilon) ; \Gamma[\epsilon / x]\left[\epsilon / w^{\prime}\right] ; \epsilon: \top^{*} \vdash \Delta[\epsilon / x]\left[\epsilon / w^{\prime}\right]
$$

By Lemma 13, $\epsilon: \top^{*}$ in the antecedent can be omitted. Apply the $E q_{1}$ rule on this sequent without $\epsilon: \top^{*}$, we finally get $\left(\epsilon, w^{\prime} \triangleright \epsilon\right) ; \Gamma[\epsilon / x] \vdash \Delta[\epsilon / x]$.

(c) $x=w^{\prime}$. The original derivation is as follows.

$$
\begin{gathered}
\Pi \\
\frac{(\epsilon, w \triangleright w) ; \Gamma[w / x] ; w: \top^{*} \vdash \Delta[w / x]}{(\epsilon, x \triangleright w) ; \Gamma ; x: \top^{*} \vdash \Delta} E q_{1}
\end{gathered}
$$

By the induction hypothesis, we have the following derivation:

Now the end sequent is equal to:

$$
(\epsilon, \epsilon \triangleright \epsilon) ; \Gamma[w / x][\epsilon / w] \vdash \Delta[w / x][\epsilon / w]
$$

$$
(\epsilon, \epsilon \triangleright \epsilon) ; \Gamma[\epsilon / x][\epsilon / w] \vdash \Delta[\epsilon / x][\epsilon / w]
$$

By using the rule $E q_{2}$ on this sequent, we derive $(\epsilon, \epsilon \triangleright w) ; \Gamma[\epsilon / x] \vdash \Delta[\epsilon / x]$.

The case where the last rule is $E q_{2}$ is similar to the case for $E q_{1}$.

If the last rule is $T^{*} L$, then the derivation to the premise of the last rule yields the new derivation.

The invertibility of $E q_{1}$ and $E q_{2}$ follows from the Substitution Lemma, as the reverse versions of these two rules are only about replacing labels. 


\section{A.5 Contraction admissibility of $L S_{B B I}$}

Lemma 16. For all structures $\Gamma, \Delta$, and labelled formula $w: A$, the following holds in $L S_{B B I}$ :

1. If there is a cut-free derivation $\Pi$ of $\Gamma ; w: A ; w: A \vdash \Delta$, then there is a cut-free derivation $\Pi{ }^{\prime}$ of $\Gamma ; w: A \vdash \Delta$ with $h t\left(\Pi^{\prime}\right) \leq h t(\Pi)$.

2. If there is a cut-free derivation $\Pi$ of $\Gamma \vdash w: A ; w: A ; \Delta$, then there is a cut-free derivation $\Pi^{\prime}$ of $\Gamma \vdash w: A ; \Delta$ with $h t\left(\Pi^{\prime}\right) \leq h t(\Pi)$.

Proof. By simultaneous induction on the height of derivations for the left and right contraction. Let $n=$ $h t(\Pi)$.

(Base case) If $n=0$, the premise is one of the conclusions of $i d, \perp L, \top R$ and $\top^{*} R$, then the contracted sequent is also the conclusion of the corresponding rules.

(Inductive case) If $n>0$, consider the last rule applied to the premise of the contraction.

(i) If the contracted formula is not principal in the last rule, then we can apply the induction hypothesis on the premise(s) of the last rule, then use the rule to get the derivation.

(ii) If the contracted formula is the principal formula of the last rule, we have several cases. For the additive rules the cases are reduced to contraction on smaller formulae, cf. [13].

For $T^{*} L$, we have the following derivation:

$$
\frac{\Pi[\epsilon / x] ; \epsilon: \top^{*} \vdash \Delta[\epsilon / x]}{\Gamma ; x: \top^{*} ; x: \top^{*} \vdash \Delta} \top^{*} L
$$

Note that the only case where $T^{*}$ is useful on the left hand side is when it is labelled with a world other than $\epsilon$. Since the substitution $[\epsilon / \epsilon]$ does not do anything to the sequent, $\Pi$ can also be the derivation for $\Gamma[\epsilon / x] \vdash \Delta[\epsilon / x]$, cf. Lemma [3], which leads to $\Gamma ; x: \top^{*} \vdash \Delta$.

For $* R$ and $-* L$, we can apply the induction hypothesis directly on the premise of the corresponding rule since the rules carry the principal formula into the premise(s).

For $* L$, we have a derivation as follows.

$$
\begin{gathered}
\Pi \\
\frac{(x, y \triangleright z) ; \Gamma ; z: A * B ; x: A ; y: B \vdash \Delta}{\Gamma ; z: A * B ; z: A * B \vdash \Delta} * L
\end{gathered}
$$

Apply the Invertibility Lemma on the premise of $* L$, we have:

$$
\begin{gathered}
\Pi^{\prime} \\
(x, y \triangleright z) ;\left(x^{\prime}, y^{\prime} \triangleright z\right) ; \Gamma ; x^{\prime}: A ; y^{\prime}: B ; x: A ; y: B \vdash \Delta
\end{gathered}
$$

The Substitution Lemma yields a derivation for $(x, y \triangleright z) ;(x, y \triangleright z) ; \Gamma ; x: A ; y: B ; x: A ; y: B \vdash \Delta$. Apply the induction hypothesis twice and admissibility of contraction on relational atoms on this sequent, to get a derivation for $(x, y \triangleright z) ; \Gamma ; x: A ; y: B \vdash \Delta$. Apply $* L$ on this sequent to get $\Gamma ; z: A * B \vdash \Delta$.

The case for $-* R$ follows similarly. We have a derivation as follows.

$$
\frac{\Pi}{(x, y \triangleright z) ; \Gamma ; x: A \vdash z: B ; y: A-* B ; \Delta}-* R
$$

The Invertibility of $-* R$ in the premise yields:

$$
(x, y \triangleright z) ;\left(x^{\prime}, y \triangleright z^{\prime}\right) ; \Gamma ; x: A ; x^{\prime}: A \vdash z: B ; z^{\prime}: B ; \Delta
$$

We obtain $(x, y \triangleright z) ;(x, y \triangleright z) ; \Gamma ; x: A ; x: A \vdash z: B ; z: B ; \Delta$ by the Substitution Lemma. Apply induction hypothesis twice, and the admissibility of contraction on relations on this sequent, to get $(x, y \triangleright z) ; \Gamma ; x: A \vdash$ $z: B \Delta$. Finally, apply $-* R$, to derive $\Gamma \vdash y: A-* B ; \Delta$ in the $n$th step. 


\section{A.6 Cut elimination}

The proof for Theorem 3 ,

Proof. By induction on the complexity of the proof in $L S_{B B I}$. We show that each application of cut can either be eliminated, or be replaced by one or more cut rules of less complexity. The argument for termination is similar to the cut-elimination proof for G3ip [13. We start to eliminate the topmost cut first, and repeat this procedure until there is no cut in the derivation. We first show that cut can be eliminated when the cut height is the lowest, i.e., at least one premise is of height 1 . Then we show that the cut height is reduced in all cases in which the cut formula is not principal in both premises of cut. If the cut formula is principal in both premises, then the cut is reduced to one or more cuts on smaller formulae or shorter derivations. Since atoms cannot be principal in logical rules, finally we can either reduce all cuts to the case where the cut formula is not principal in both premises, or reduce those cuts on compound formulae until their cut heights are minimal and then eliminate those cuts.

(Base case) If at least one premise of the $c u t$ rule is $i d, \perp L, \top R$, or $\top^{*} R$, we consider the following cases:

1. The left premise of cut is an application of $i d$, and the cut formula is not principal, then the derivation is transformed as follows.

$$
\begin{aligned}
& \frac{{ }^{\Gamma ; y: B \vdash y: B ; x: A ; \Delta} i d \quad \Gamma^{\prime} ; x: A \vdash \Delta^{\prime}}{\Gamma ; \Gamma^{\prime} ; y: B \vdash y: B ; \Delta ; \Delta^{\prime}} \text { cut }{ }^{\rightsquigarrow} \\
& \overline{\Gamma ; \Gamma^{\prime} ; y: B \vdash y: B ; \Delta ; \Delta^{\prime}} i d
\end{aligned}
$$

The same transformation works for $\perp L, \top R, \top^{*} R$ in this case.

2. The left premise of cut is an application of $i d$, and the cut formula is principal, then the derivation is transformed as follows.

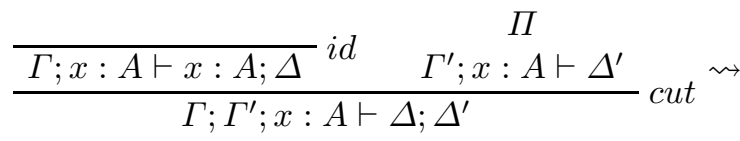

$$
\begin{aligned}
& \Pi \\
& \Gamma^{\prime} ; x: A \vdash \Delta^{\prime} \\
& \overline{\Gamma ;} \overline{\Gamma^{\prime}} ; x: \overline{A \vdash} \overline{\Delta ;} \overline{\Delta^{\prime}} \text { Lemma } 2
\end{aligned}
$$

3. The left premise of cut is an application of $T R$, and the cut formula is principal, then the derivation is transformed as follows.

$$
\begin{aligned}
& \frac{\frac{\Pi}{\Gamma \vdash x: \top ; \Delta} \top R \quad \Gamma^{\prime} ; x: \top \vdash \Delta^{\prime}}{\Gamma ; \Gamma^{\prime} \vdash \Delta ; \Delta^{\prime}} \text { cut } \rightsquigarrow \\
& \Pi^{\prime} \\
& -\overline{\Gamma ;} \bar{\Gamma}^{\prime} \vdash-\frac{\Delta^{\prime}}{\vdash}-\bar{\Delta} ; \Delta^{\prime} \text { Lemma } 2
\end{aligned}
$$

As $x$ : $\top$ cannot be a principal formula in the antecedent, by Lemma 14 there is a derivation $\Pi^{\prime}$ of $\Gamma^{\prime} \vdash \Delta^{\prime}$.

The same holds for $T^{*} R$.

4. The right premise of cut is an application of $i d, \perp L, \top R$ or $\top^{*} R$, and the cut formula is not principal. This case is similar to case 1 .

5. The right premise of $c u t$ is an application of $i d$, and the cut formula is principal. This case is similar to case 2.

6. The right premise of cut is an application of $\perp L$, and the cut formula is principal. This case is similar to case 3 . 
(Inductive case) If both premises are not in one of the base cases, we distinguish three cases here: the cut formula is not principal in the left premises; the cut formula is only principal in the left premise; and the cut formula is principal in both premises.

1. The cut formula is not principal in the left premise. Suppose the left premise ends with a rule $r$.

(a) If $r$ is $T^{*} L$, w.l.o.g. we assume the label of the principal formula is $y$ (which might be equal to $x$ ). The original derivation is as follows.

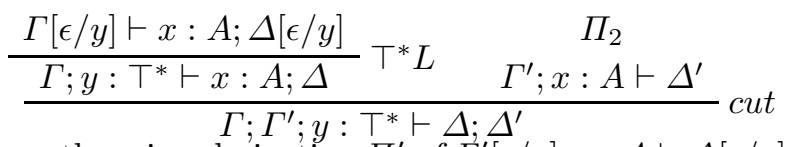

By the Substitution lemma, there is a derivation $\Pi_{2}^{\prime}$ of $\Gamma^{\prime}[\epsilon / y] ; x: A \vdash \Delta[\epsilon / y]$. Thus we can transform the derivation into the following:

$$
\begin{aligned}
& \Pi_{1} \quad \Pi_{2}^{\prime} \\
& \frac{\Gamma[\epsilon / y] \vdash x: A ; \Delta[\epsilon / y] \quad \Gamma^{\prime}[\epsilon / y] ; x: A \vdash \Delta^{\prime}[\epsilon / y]}{\frac{\Gamma[\epsilon / y] ; \Gamma^{\prime}[\epsilon / y] \vdash \Delta[\epsilon / y] ; \Delta^{\prime}[\epsilon / y]}{\Gamma ; \Gamma^{\prime} ; y: \top^{*} \vdash \Delta ; \Delta^{\prime}}} \top^{*} L
\end{aligned}
$$

If $x=y$ in the original derivation, then the new derivation cuts on $\epsilon$ : $A$ instead. As substitution is height preserving, the cut height in this case is reduced as well.

(b) If $r$ is $E q_{1}$, and the label $x$ of the principal formula is not equal to $w^{\prime}$, the original derivation is as follows.

$$
\begin{aligned}
& \Pi_{1}
\end{aligned}
$$

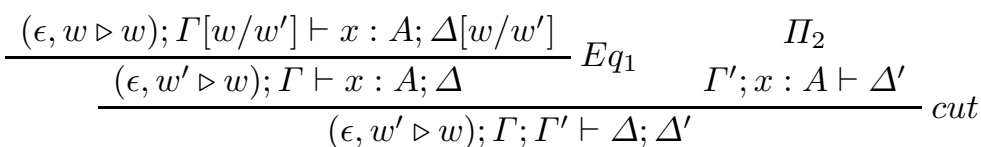

This cut is reduced in the same way as the $\top^{*} L$ case, where we get $\Pi_{2}^{\prime}$ from the Substitution Lemma:

$$
\begin{aligned}
& \Pi_{1}
\end{aligned}
$$

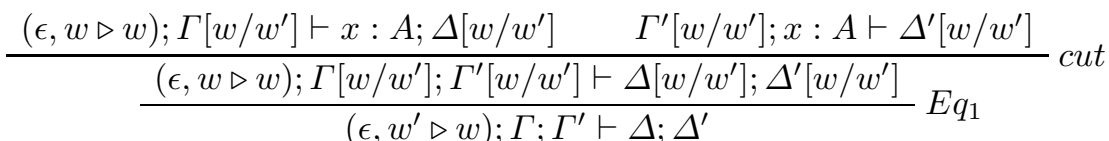

If $x=w^{\prime}$, then we cut on $w: A$ instead in the reduced version.

(c) If $r$ is $E q_{2}$, the procedure follows similarly as the case for $E q_{1}$ above.

(d) If $r$ is a unary inference except for $\top_{\Pi_{1}}^{*} L, E q_{1}$, and $E q_{2}$, then the original derivation is as follows.

$$
\frac{\frac{\Gamma_{1} \vdash x: A ; \Delta_{1}}{\Gamma \vdash x: A ; \Delta} r \quad \Gamma^{\prime} ; x: A \vdash \Delta^{\prime}}{\Gamma ; \Gamma^{\prime} \vdash \Delta ; \Delta^{\prime}} \text { cut }
$$

Then we can delay the application of cut as follows. $\Pi_{1}$

$$
\frac{\Gamma_{1} \vdash x: A ; \Delta_{1} \quad \Gamma^{\prime} ; x: A \vdash \Delta^{\prime}}{\frac{\Gamma_{1} ; \Gamma^{\prime} \vdash \Delta_{1} ; \Delta^{\prime}}{\Gamma ; \Gamma^{\prime} \vdash \Delta ; \Delta^{\prime}} r} \text { cut }
$$

Note that as all our rules except $\top^{*} L, E q_{1}$, and $E q_{2}$ do not modify side structures, $\Gamma^{\prime}$ and $\Delta^{\prime}$ in the premise of $r$ are not changed. The complexity of the original cut is $\left(|x: A|,\left|\Pi_{1}\right|+1+\left|\Pi_{2}\right|\right)$, whereas the complexity of the new cut is $\left(|x: A|,\left|\Pi_{1}\right|+\left|\Pi_{2}\right|\right)$, so the cut height reduces.

(e) If $r$ is a binary inference, we can transform the derivation similarly.

$$
\begin{aligned}
& \Pi_{1} \quad \Pi_{2}
\end{aligned}
$$

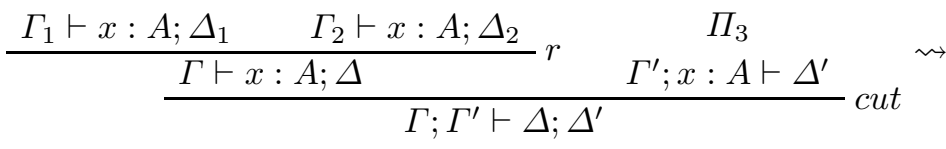

$$
\begin{aligned}
& \Pi_{1} \quad \Pi_{3} \quad \Pi_{2} \quad \Pi_{3} \\
& \frac{\Gamma_{1} \vdash x: A ; \Delta_{1} \quad \Gamma^{\prime} ; x: A \vdash \Delta^{\prime}}{\frac{\Gamma_{1} ; \Gamma^{\prime} \vdash \Delta_{1} ; \Delta^{\prime}}{\Gamma} \text { cut } \frac{\Gamma_{2} \vdash x: A ; \Delta_{2}}{\Gamma_{2} ; \Gamma^{\prime} \vdash \Delta_{2} ; \Delta^{\prime}}} r
\end{aligned}
$$


The complexity of the original cut is $\left(|x: A|, \max \left(\left|\Pi_{1}\right|,\left|\Pi_{2}\right|\right)+1+\left|\Pi_{3}\right|\right)$, and that of the new two cuts are $\left(|x: A|,\left|\Pi_{1}\right|+\left|\Pi_{3}\right|\right)$ and $\left(|x: A|,\left|\Pi_{2}\right|+\left|\Pi_{3}\right|\right)$ respectively. Thus the cut heights are reduced.

2. The cut formula is only principal in the left premise. We only consider the last rule in the right branch. The proof of this case is symmetric to those in Case 1.

3. The cut formula is principal in both premises. We do a case analysis on the main connective of the cut formula. If the main connective is additive, then there is no need to substitute any labels.

For $\wedge$,

$$
\begin{aligned}
& \Pi_{1} \quad \Pi_{2} \quad \Pi_{3}
\end{aligned}
$$

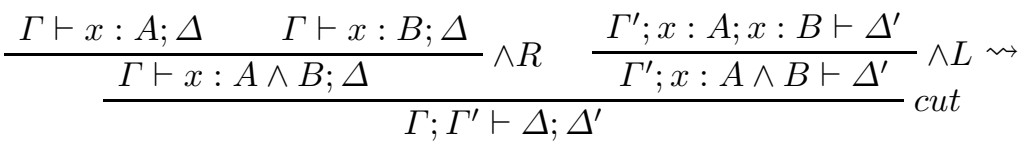

$$
\begin{aligned}
& \Pi_{2} \quad \Pi_{3}
\end{aligned}
$$

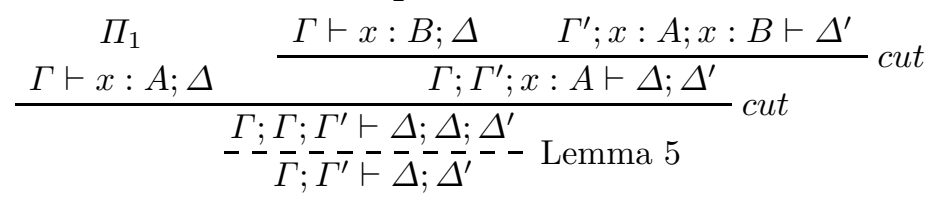

For $\rightarrow$,

$$
\begin{aligned}
& \Pi_{1} \quad \Pi_{2} \quad \Pi_{3} \\
& \frac{\frac{\Gamma^{\prime} ; x: A \vdash x: B ; \Delta^{\prime}}{\Gamma^{\prime} \vdash x: A \rightarrow B ; \Delta^{\prime}} \rightarrow R \quad \frac{\Gamma \vdash x: A ; \Delta \quad \Gamma ; x: B \vdash \Delta}{\Gamma ; x: A \rightarrow B \vdash \Delta}}{\Gamma ; \Gamma^{\prime} \vdash \Delta ; \Delta^{\prime}} \text { cut } \rightarrow L \rightsquigarrow \\
& \Pi_{1} \quad \Pi_{3}
\end{aligned}
$$

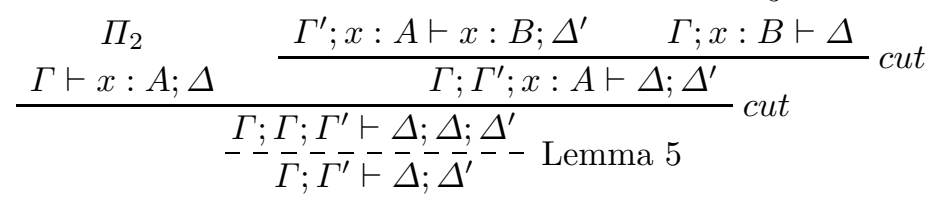

For both $\wedge$ and $\rightarrow$, cut is reduced to applications on smaller formulae, therefore the complexity of the cut reduces.

There is an asymmetry in the rules for $T^{*}$. That is, the left rule for $\top^{*}$ requires that the label $w$ of $\top^{*}$ cannot be $\epsilon$, whereas the right rule for $T^{*}$ restricts the label of $T^{*}$ to be $\epsilon$ only. As a consequence, when the cut formula is $T^{*}$, it cannot be the principal formula of both premises at the same time. Therefore the cases for $T^{*}$ are handled in the proof above.

When the main connective of the cut formula is $*$ or $-*$, the case is more complicated. For $*$, we have the following two derivations as the premises of the cut rule:

$$
\frac{\Pi_{1}}{(x, y \triangleright z) ; \Gamma \vdash x: A ; z: A * B ; \Delta \quad(x, y \triangleright z) ; \Gamma \vdash y: B ; z: A * B ; \Delta} * R
$$

and

$$
\begin{aligned}
& \Pi_{3} \\
& \frac{\left(x^{\prime}, y^{\prime} \triangleright z\right) ; \Gamma^{\prime} ; x^{\prime}: A ; y^{\prime}: B \vdash \Delta^{\prime}}{\Gamma^{\prime} ; z: A * B \vdash \Delta^{\prime}} *
\end{aligned}
$$

And the cut rule gives the end sequent $(x, y \triangleright z) ; \Gamma ; \Gamma^{\prime} \vdash \Delta ; \Delta^{\prime}$. The complexity of this cut is $(\mid A *$ $\left.B\left|, \max \left(\left|\Pi_{1}\right|,\left|\Pi_{2}\right|\right)+1+\right| \Pi_{3} \mid+1\right)$.

We use several cuts with less complexity to derive $(x, y \triangleright z) ; \Gamma ; \Gamma^{\prime} \vdash \Delta ; \Delta^{\prime}$ as follows.

Firstly, 


$$
\begin{aligned}
& \Pi_{3} \\
& \frac{\Pi_{1}}{(x, y \triangleright z) ; \Gamma \vdash x: A ; z: A * B ; \Delta} \frac{\frac{\left(x^{\prime}, y^{\prime} \triangleright z\right) ; \Gamma^{\prime} ; x^{\prime}: A ; y^{\prime}: B \vdash \Delta^{\prime}}{\Gamma^{\prime} ; z: A * B \vdash \Delta^{\prime}}}{(x, y \triangleright z) ; \Gamma ; \Gamma^{\prime} \vdash x: A ; \Delta ; \Delta^{\prime}} \text { cut }
\end{aligned}
$$

The complexity of this cut is $\left.\left(|A * B|,\left|\Pi_{1}\right|+\left|\Pi_{3}\right|+1\right)\right)$, thus is less than the original cut. The second cut works similarly.

$$
\frac{\Pi_{2}}{(x, y \triangleright z) ; \Gamma \vdash y: B ; z: A * B ; \Delta} \begin{gathered}
\Pi_{3} \\
(x, y \triangleright z) ; \Gamma ; \Gamma^{\prime} \vdash y: B ; \Delta ; \Delta^{\prime}
\end{gathered}
$$

The third cut works on a smaller formula.

$$
\frac{(x, y \triangleright z) ; \Gamma ; \Gamma^{\prime} \vdash x: A ; \Delta ; \Delta^{\prime} \quad(x, y \triangleright z) ; \Gamma^{\prime} ; x: A ; y: B \vdash \Delta^{\prime}}{(x, y \triangleright z) ;(x, y \triangleright z) ; \Gamma ; \Gamma^{\prime} ; \Gamma^{\prime} ; y: B \vdash \Delta ; \Delta^{\prime} ; \Delta^{\prime}} c u t
$$

The cut formula is $x: A$, thus the complexity of this cut is less regardless of the height of the derivations. Note that in the $\Pi_{3}$ branch, the $* L$ rule requires that the relation $\left(x^{\prime}, y^{\prime} \triangleright z\right)$ is newly created, so $x^{\prime}$ and $y^{\prime}$ cannot be $\epsilon$ and they cannot be in $\Gamma^{\prime}$ or $\Delta^{\prime}$. Therefore we are allowed to use the substitution lemma to get a derivation $\Pi_{3}^{\prime}$ of $(x, y \triangleright z) ; \Gamma^{\prime} ; x: A ; y: B \vdash \Delta^{\prime}$ by just substituting $x^{\prime}$ for $x$ and $y^{\prime}$ for $y$.

Finally we cut on another smaller formula $y: B$.

$$
\frac{(x, y \triangleright z) ; \Gamma ; \Gamma^{\prime} \vdash y: B ; \Delta ; \Delta^{\prime} \quad(x, y \triangleright z) ;(x, y \triangleright z) ; \Gamma ; \Gamma^{\prime} ; \Gamma^{\prime} ; y: B \vdash \Delta ; \Delta^{\prime} ; \Delta^{\prime}}{(x, y \triangleright z) ;(x, y \triangleright z) ;(x, y \triangleright z) ; \Gamma ; \Gamma ; \Gamma^{\prime} ; \Gamma^{\prime} ; \Gamma^{\prime} \vdash \Delta ; \Delta ; \Delta^{\prime} ; \Delta^{\prime} ; \Delta^{\prime}} c u t
$$

The complexity of this cut is less than the original cut. We then apply the admissibility of contraction to derive $(x, y \triangleright z) ; \Gamma ; \Gamma^{\prime} \vdash \Delta ; \Delta^{\prime}$.

The case for $-*$ is similar. The two premises in the original cut are as follows.

$$
\begin{aligned}
& \Pi_{1} \\
& \frac{\left(x^{\prime}, y \triangleright z^{\prime}\right) ; \Gamma^{\prime} ; x^{\prime}: A \vdash z^{\prime}: B ; \Delta^{\prime}}{\Gamma^{\prime} \vdash y: A-* B ; \Delta^{\prime}}-* R
\end{aligned}
$$

and

$$
\begin{aligned}
& \Pi_{2} \quad \Pi_{3} \\
& \frac{(x, y \triangleright z) ; \Gamma ; y: A-* B \vdash x: A ; \Delta \quad(x, y \triangleright z) ; \Gamma ; y: A-* B ; z: B \vdash \Delta}{(x, y \triangleright z) ; \Gamma ; y: A-* B \vdash \Delta}-* L
\end{aligned}
$$

And the cut rule yields the end sequent $(x, y \triangleright z) ; \Gamma ; \Gamma^{\prime} \vdash \Delta ; \Delta^{\prime}$. We use two cuts on the same formula, but with smaller derivation height.

$$
\begin{aligned}
& \Pi_{1}
\end{aligned}
$$

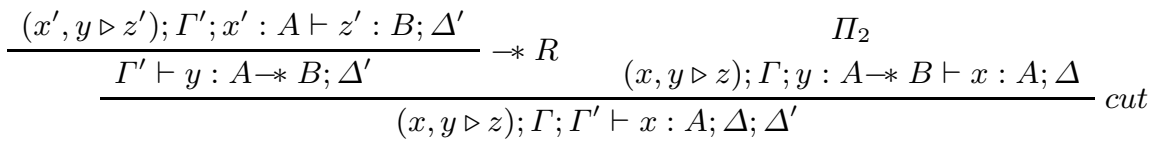

$$
\begin{aligned}
& \Pi_{1}
\end{aligned}
$$

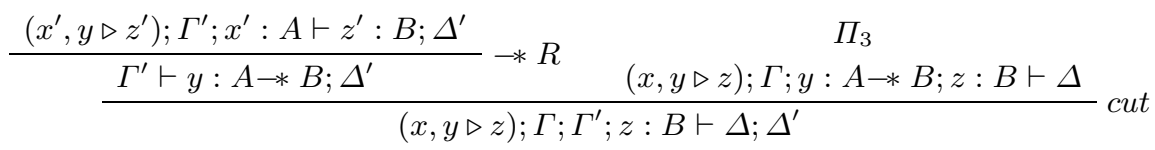

Then we cut on a smaller formula $x: A$.

$$
\frac{(x, y \triangleright z) ; \Gamma ; \Gamma^{\prime} \vdash x: A ; \Delta ; \Delta^{\prime} \quad(x, y \triangleright z) ; \Gamma^{\prime} ; x: A \vdash z: B ; \Delta^{\prime}}{(x, y \triangleright z) ;(x, y \triangleright z) ; \Gamma ; \Gamma^{\prime} ; \Gamma^{\prime} \vdash z: B ; \Delta ; \Delta^{\prime} ; \Delta^{\prime}} c u t
$$


Again, in the original derivation, $x^{\prime}$ and $z^{\prime}$ are fresh in the premise of $-* R$ rule, thus by the Substitution Lemma we can have a derivation $\Pi_{1}^{\prime}$ of the sequent $(x, y \triangleright z) ; \Gamma^{\prime} ; x: A \vdash z: B ; \Delta^{\prime}$, with $x^{\prime}$ substituted to $x$ and $z^{\prime}$ substituted to $z$.

Then we cut on $z: B$.

$$
\frac{(x, y \triangleright z) ;(x, y \triangleright z) ; \Gamma ; \Gamma^{\prime} ; \Gamma^{\prime} \vdash z: B ; \Delta ; \Delta^{\prime} ; \Delta^{\prime} \quad(x, y \triangleright z) ; \Gamma ; \Gamma^{\prime} ; z: B \vdash \Delta ; \Delta^{\prime}}{(x, y \triangleright z) ;(x, y \triangleright z) ;(x, y \triangleright z) ; \Gamma ; \Gamma ; \Gamma^{\prime} ; \Gamma^{\prime} ; \Gamma^{\prime} \vdash \Delta ; \Delta ; \Delta^{\prime} ; \Delta^{\prime} ; \Delta^{\prime}} c u t
$$

In the end we use the theorem of admissibility of contraction to obtain the required sequent $(x, y \triangleright$ $z) ; \Gamma ; \Gamma^{\prime} \vdash \Delta ; \Delta^{\prime}$.

\section{A.7 Permutation of structural rules in $L S_{B B I}$}

Proof for Lemma 6.

Proof. To prove this lemma, we need to show that if a derivation involves the structural rules, we can always apply them exactly before $* R$ and $-* L$, or before zero-premise rules. We show this by an induction on the height of the derivation. Since we do not permute structural rules through zero-premise rules, the proof in the base case and the inductive step are essentially the same. Here we give some examples of the permutations. Assuming the lemma holds up to any derivation of height $n-1$, consider a derivation of height $n$.

1. Permute the application of $E q_{1}$ or $E q_{2}$ through non-zero-premise logical rules except for $* R$ and $-* L$. Here we give some examples, the rest are similar.

(a) Permute $E q_{2}$ through additive logical rules is trivial, this is exemplified by $\wedge L$, assuming the label of the principal formula is modified by the $E q_{2}$ application. The original derivation is as follows.

$$
\frac{(\epsilon, \epsilon \triangleright \epsilon) ; \Gamma[\epsilon / w] ; \epsilon: A ; \epsilon: B \vdash \Delta[\epsilon / w]}{\frac{(\epsilon, \epsilon \triangleright \epsilon) ; \Gamma[\epsilon / w] ; \epsilon: A \wedge B \vdash \Delta[\epsilon / w]}{(\epsilon, \epsilon \triangleright w) ; \Gamma ; w: A \wedge B \vdash \Delta} \wedge L} E q_{2}
$$

The derivation is changed to the following:

$$
\frac{(\epsilon, \epsilon \triangleright \epsilon) ; \Gamma[\epsilon / w] ; \epsilon: A ; \epsilon: B \vdash \Delta[\epsilon / w]}{\frac{(\epsilon, \epsilon \triangleright w) ; \Gamma ; w: A ; w: B \vdash \Delta}{(\epsilon, \epsilon \triangleright w) ; \Gamma ; w: A \wedge B \vdash \Delta} \wedge L} E q_{2}
$$

(b) Permute $E q_{1}$ through $\top^{*} L$, assuming the label of principal formula is $w$. The derivation is as follows.

$$
\Pi
$$

$$
\frac{\frac{(\epsilon, \epsilon \triangleright \epsilon) ; \Gamma\left[w / w^{\prime}\right][\epsilon / w] \vdash \Delta\left[w / w^{\prime}\right][\epsilon / w]}{(\epsilon, w \triangleright w) ; \Gamma\left[w / w^{\prime}\right] ; w: \top^{*} \vdash \Delta\left[w / w^{\prime}\right]}}{\left(\epsilon, w^{\prime} \triangleright w\right) ; \Gamma ; w^{\prime}: \top^{*} \vdash \Delta} \top^{*} L
$$

We modify the derivation as follows.

$$
\begin{aligned}
& \text { II } \\
& \frac{(\epsilon, \epsilon \triangleright \epsilon) ; \Gamma\left[\epsilon / w^{\prime}\right][\epsilon / w] \vdash \Delta\left[\epsilon / w^{\prime}\right][\epsilon / w]}{\frac{(\epsilon, \epsilon \triangleright w) ; \Gamma\left[\epsilon / w^{\prime}\right] \vdash \Delta\left[\epsilon / w^{\prime}\right]}{\left(\epsilon, w^{\prime} \triangleright w\right) ; \Gamma ; w^{\prime}: \top^{*} \vdash \Delta} \top^{*} L} E q_{2}
\end{aligned}
$$

Notice that the premises of the two derivations below $\Pi$ are exactly the same. The application of $E q_{1}$ in the original derivation is changed to an application of $E q_{2}$ in the modified derivation. However, this does not break the proof, as the induction hypothesis ensures that either of them can be permuted upwards.

Also, the label of principal formula in the rule $T^{*} L$ cannot be the one that is replaced in the rule $E q_{2}$ below it, this is the reason we do not exemplify this situation using $E q_{2}$.

(c) Permute $E q_{2}$ through $* L$, assuming the label of principal formula is $z$, and it is modified by the $E q_{2}$ application.

$$
\begin{aligned}
& \text { II } \\
& \frac{(x, y \triangleright \epsilon) ;(\epsilon, \epsilon \triangleright \epsilon) ; \Gamma[\epsilon / z] ; x: A ; y: B \vdash \Delta[\epsilon / z]}{\frac{(\epsilon, \epsilon \triangleright \epsilon) ; \Gamma[\epsilon / z] ; \epsilon: A * B \vdash \Delta[\epsilon / z]}{(\epsilon, \epsilon \triangleright z) ; \Gamma ; z: A * B \vdash \Delta} E q_{2}} * L
\end{aligned}
$$


Since $x$ and $y$ are fresh labels, they will not be affected by $E q_{2}$. Thus the derivation can be changed to the following:

$$
\begin{aligned}
& \Pi \\
& \frac{(x, y \triangleright \epsilon) ;(\epsilon, \epsilon \triangleright \epsilon) ; \Gamma[\epsilon / z] ; x: A ; y: B \vdash \Delta[\epsilon / z]}{\frac{(x, y \triangleright z) ;(\epsilon, \epsilon \triangleright z) ; \Gamma ; x: A ; y: B \vdash \Delta}{(\epsilon, \epsilon \triangleright y) ; \Gamma ; z: A * B \vdash \Delta} * L} E q_{2}
\end{aligned}
$$

Since $E q_{1}$ and $E q_{2}$ only globally replaces labels, their action can be safely delayed through all the rules other than $* R$ and $-* L$. The applications of these two rules after the last $* R$ or $* L$ will be delayed until the zero-premise rule is necessary.

2. Permute the applications of $E, U, A$, and $A_{C}$ through non-zero premise logical rules other than $* R$ and $-* L$. Again, we give some examples, the rest are similar.

(a) Permute $E$ through $T^{*} L$, assuming the label of the principal formula is $y$. The original derivation runs as follows.

The new derivation is as follows.

$$
\begin{gathered}
\Pi \\
\frac{(\epsilon, x \triangleright z) ;(x, \epsilon \triangleright z) ; \Gamma[\epsilon / y] \vdash \Delta[\epsilon / y]}{(y, x \triangleright z) ;(x, y \triangleright z) ; \Gamma ; y: \top^{*} \vdash \Delta} \\
(x, y \triangleright z) ; \Gamma ; y: \top^{*} \vdash \Delta
\end{gathered}
$$

$$
\frac{(\epsilon, x \triangleright z) ;(x, \epsilon \triangleright z) ; \Gamma[\epsilon / y] \vdash \Delta[\epsilon / y]}{\frac{(x, \epsilon \triangleright z) ; \Gamma[\epsilon / y] \vdash \Delta[\epsilon / y]}{(x, y \triangleright z) ; \Gamma ; y: \top^{*} \vdash \Delta} \top^{*} L} E
$$

This shows that if the logical rule only does substitution, delaying the application of structural rules makes no difference.

(b) Permute $U$ through $* L$, assuming the label of the principal formula is $z$. The original derivation is as follows.

The new derivation is as follows.

$$
\frac{(x, y \triangleright z) ;(z, \epsilon \triangleright z) ; \Gamma ; x: A ; y: B \vdash \Delta}{\frac{(z, \epsilon \triangleright z) ; \Gamma ; z: A * B \vdash \Delta}{\Gamma ; z: A * B \vdash \Delta} U} * L
$$

$$
\frac{(z, \epsilon \triangleright z) ;(x, y \triangleright z) ; \Gamma ; x: A ; y: B \vdash \Delta}{\frac{(x, y \triangleright z) ; \Gamma ; z: A * B \vdash \Delta}{\Gamma ; z: A * B \vdash \Delta} * L} U
$$

Since the labels $x$ and $y$ are all fresh labels, it is safe to change the order to rule applications as above.

Additive logical rules are totally independent on the relational atoms, so those cases are similar as the one shown above, except that those rules do not add relational atoms to the sequent.

\section{A.8 Soundness of $L S_{B B I}^{e}$}

Theorem 8. If there is a derivation $\Pi$ for a sequent $\Gamma \vdash \Delta$ in $L S_{B B I}^{e}$, then there is a derivation $\Pi^{\prime}$ for the same sequent in $L S_{B B I}$.

Proof. By induction on the height $n$ of $\Pi$.

1. Base case: $n=1$. In this case the only rule must be a zero-premise rule. If the rule is $\perp L$ or $\top R$, then we can use the same rule in $L S_{B B I}$, since they are the same. Otherwise, suppose the rule is $i d$, then $\Pi$ reads as follows.

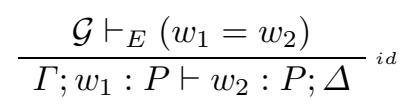

Since $\mathcal{G} \vdash_{E}\left(w_{1}=w_{2}\right)$ is true, there is a sequence $\sigma$ of $E q_{1}, E q_{2}$ applications such that $\mathcal{S}(\mathcal{G}, \sigma)$ is defined and $w_{1} \theta=w_{2} \theta$, where $\theta=\operatorname{subst}(\sigma)$. Therefore we can construct $\Pi^{\prime}$ are follows. 


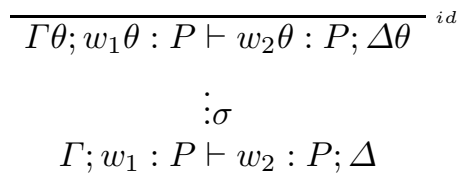

If the rule is $\top^{*} R, \Pi$ is:

$$
\frac{\mathcal{G} \vdash_{E}(w=\epsilon)}{\Gamma \vdash w: \top^{*} ; \Delta} \top^{*} R
$$

We construct $\Pi^{\prime}$ similarly, as $w \theta=\epsilon$ after the application of $\sigma$.

$$
\begin{aligned}
& {\overline{\Gamma \theta \vdash w \theta: \top^{*} ; \Delta \theta}}^{{ }^{*}{ }_{R}} \\
& \vdots \sigma \\
& \Gamma \vdash w: \top^{*} ; \Delta
\end{aligned}
$$

2. Inductive cases: suppose every sequent that is derivable in $L S_{B B I}^{e}$ with height less than $n$ is also derivable in $L S_{B B I}$, consider a $L S_{B B I}^{e}$ derivation of height $n$. We do a case analysis on the bottom rule in the derivation.

(a) If the rule is $\wedge L, \wedge R, \rightarrow L, \rightarrow R, * L, * R, E$ or $U$, we can use the same rule in $L S_{B B I}$, since nothing is changed.

(b) If the rule is $T^{*} L$, then $\Pi$ must be the following:

$$
\frac{(\epsilon, w \triangleright \epsilon) ; \Gamma \vdash \Delta}{\Gamma ; w: \top^{*} \vdash \Delta} \top^{*}{ }_{L}
$$

By the induction hypothesis, $(\epsilon, w \triangleright \epsilon) ; \Gamma \vdash \Delta$ is derivable in $L S_{B B I}$. Applying Lemma 1 (substitution for labels in $\left.L S_{B B I}\right)$ with $[\epsilon / w]$, we obtain $(\epsilon, \epsilon \triangleright \epsilon) ; \Gamma[\epsilon / w] \vdash \Delta[\epsilon / w]$. Thus we construct $\Pi^{\prime}$ as follows.

$$
\Pi_{1}^{\prime}
$$

$$
\frac{\frac{(\epsilon, \epsilon \triangleright \epsilon) ; \Gamma[\epsilon / w] \vdash \Delta[\epsilon / w]}{(w, \epsilon \triangleright w) ; \Gamma ; w: \top^{*} \vdash \Delta}}{\Gamma ; w: \top^{*} \vdash \Delta}{ }^{*}{ }_{L}
$$

(c) If the rule is $* R, \Pi$ runs as follows.

$$
\frac{\begin{array}{c}
\Pi_{1} \\
\Pi_{2}
\end{array}}{\left(x, y \triangleright z^{\prime}\right) ; \Gamma \vdash x: A ; z: A * B ; \Delta \quad\left(x, y \triangleright z^{\prime}\right) ; \Gamma \vdash y: B ; z: A * B ; \Delta} * R
$$

The condition on the $* R$ rule is $\mathcal{G} \vdash_{E}\left(z=z^{\prime}\right)$. Let $\sigma$ be the sequence of $E q_{1}, E q_{2}$ applications such that $\mathcal{S}(\mathcal{G}, \sigma)$ is defined and, $z \theta=z^{\prime} \theta$ holds, where $\theta=\operatorname{subst}(\sigma)$. Also, applying the induction hypothesis on $\Pi_{1}$ and $\Pi_{2}$, we obtain the $L S_{B B I}$ derivations for each branch respectively. Then with the help of the Substitution lemma, we get two derivations as follows. Note that we use dashed lines when applying the Substitution lemmas.

$$
\begin{aligned}
& \Pi_{1}^{\prime} \\
& \left(x, y \triangleright z^{\prime}\right) ; \Gamma \vdash x: A ; z: A * B ; \Delta \\
& \overline{\left(x \theta, y \theta \triangleright z^{\prime} \theta\right)} ; \bar{\Gamma} \bar{\theta} \bar{\vdash} \bar{x} \bar{A}: \bar{A} ; \bar{y}: \bar{A} * \bar{B} ; \bar{\Delta} \bar{\theta} \text { Lemma回 }
\end{aligned}
$$

and

$$
\begin{aligned}
& \Pi_{2}^{\prime} \\
& \left(x, y \triangleright z^{\prime}\right) ; \Gamma \vdash y: B ; z: A * B ; \Delta
\end{aligned}
$$

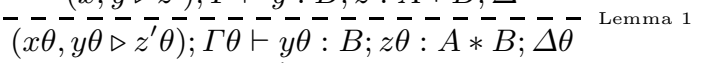

Then we can apply $* R$ and obtain $\left(x \theta, y \theta \triangleright z^{\prime} \theta\right) ; \Gamma \vdash z \theta: A * B ; \Delta \theta$. Then by applying $\sigma$ we obtain the end sequent as follows.

$$
\begin{gathered}
\left(x \theta, y \theta \triangleright z^{\prime} \theta\right) ; \Gamma \vdash z \theta: A * B ; \Delta \theta \\
\vdots \sigma \\
\left(x, y \triangleright z^{\prime}\right) ; \Gamma \vdash z: A * B ; \Delta
\end{gathered}
$$

The case for $-* L$ is treated similarly.

(d) If the rule is $A$, the treatment for the equality entailment is the same. $\Pi$ is in the following form: 


$$
\frac{(u, w \triangleright z) ;(y, v \triangleright w) ;(x, y \triangleright z) ;\left(u, v \triangleright x^{\prime}\right) ; \Gamma \vdash \Delta \quad \mathcal{G} \vdash_{E}\left(x=x^{\prime}\right)}{(x, y \triangleright z) ;\left(u, v \triangleright x^{\prime}\right) ; \Gamma \vdash \Delta} A
$$

Let $\mathcal{S}(\mathcal{G}, \sigma)$ yield $x \theta=x^{\prime} \theta$, where $\theta=\operatorname{subst}(\sigma)$, we obtain $\Pi^{\prime}$ as follows.

$$
\Pi_{1}^{\prime}
$$

$$
\begin{aligned}
& (u, w \triangleright z) ;(y, v \triangleright w) ;(x, y \triangleright z) ;\left(u, v \triangleright x^{\prime}\right) ; \Gamma \vdash \Delta
\end{aligned}
$$

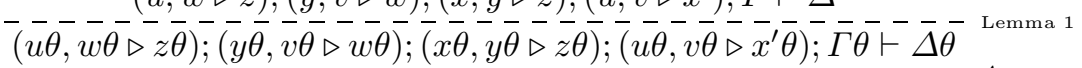

$$
\begin{aligned}
& (x \theta, y \theta \triangleright z \theta) ;\left(u \theta, v \theta \triangleright x^{\prime} \theta\right) ; \Gamma \theta \vdash \Delta \theta \\
& \vdots \sigma \\
& (x, y \triangleright z) ;\left(u, v \triangleright x^{\prime}\right) ; \Gamma \vdash \Delta
\end{aligned}
$$

The case for $A_{C}$ is similar.

\section{A.9 Completeness of $L S_{B B I}^{e}$}

To prove the completeness of $L S_{B B I}^{e}$, firstly we add $E q_{1}$ and $E q_{2}$ in $L S_{B B I}^{e}$ and show that the resultant system has the same power as $L S_{B B I}$. Then we prove the admissibility of $E q_{1}$ and $E q_{2}$ in $L S_{B B I}^{e}$.

Lemma 17. If a sequent $\Gamma \vdash \Delta$ is derivable in $L S_{B B I}$, then it is derivable in $L S_{B B I}^{e}+E q_{1}+E q_{2}$.

Proof. By induction on the height of the $L S_{B B I}$ derivation. Since with $E q_{1}$ and $E q_{2}$, most of other rules become identical, the only non-trivial case is $\top^{*} L$.

In $L S_{B B I}$, the derivation runs as follows.

$$
\frac{\Pi}{\Gamma[\epsilon / w] \vdash \Delta[\epsilon / w]} \frac{{ }^{*}{ }_{L}}{\Gamma ; w: \top^{*} \vdash \Delta}
$$

By the induction hypothesis, there is a derivation for $\Gamma[\epsilon / w] \vdash \Delta[\epsilon / w]$ in $L S_{B B I}^{e}+E q_{1}+E q_{2}$. Therefore we construct the derivation as follows.

$$
\begin{gathered}
\Pi^{\prime} \\
\frac{\Gamma[\epsilon / w] \vdash \Delta[\epsilon / w]}{\frac{(\epsilon, \epsilon \triangleright \bar{\epsilon}) ; \bar{\Gamma}[\bar{\epsilon} / \bar{w}] \vdash \bar{\Delta}[\bar{\epsilon} / \bar{w}]}{\Gamma ; w \triangleright \epsilon) ; \Gamma \vdash \Delta}} \mathrm{T}^{*}{ }_{L} \\
q_{1}
\end{gathered}
$$

Lemma 18. If $\mathcal{G}[x / y] ;(\epsilon, x \triangleright x) \vdash_{E}\left(w_{1}[x / y]=w_{2}[x / y]\right)$ then $\mathcal{G} ;(\epsilon, y \triangleright x) \vdash_{E}\left(w_{1}=w_{2}\right)$.

Proof. Let $\mathcal{G}^{\prime}=\mathcal{G} ;(\epsilon, y \triangleright x)$ and $\mathcal{S}\left(\mathcal{G}^{\prime}[x / y], \sigma\right)$ yield $\left(w_{1}[x / y] \theta=w_{2}[x / y] \theta\right)$, we show that $\mathcal{G}^{\prime} \vdash_{E}(x=y)$ by following:

$$
\begin{gathered}
\mathcal{G}^{\prime}[x / y] \theta \vdash_{E}\left(w_{1}[x / y] \theta=w_{2}[x / y] \theta\right) \\
\frac{\mathcal{G}^{\prime}[x / y] \vdash_{E}\left(w_{1}[x / y]=w_{2}[x / y]\right)}{\mathcal{G} ;(\epsilon, y \triangleright x) \vdash_{E}(x=y)} E q_{1}
\end{gathered}
$$

Now we show that $E q_{1}$ is admissible in $L S_{B B I}^{e}$.

Lemma 19. If $(\epsilon, x \triangleright x) ; \Gamma[x / y] \vdash \Delta[x / y]$ is derivable in $L S_{B B I}^{e}$, then $(\epsilon, y \triangleright x) ; \Gamma \vdash \Delta$ is derivable in LS ${ }_{B B I}^{e}$.

Proof. We show that $E q_{1}$ can always permute up through all other rules, and eventually disappear when it hits the zero-premise rule. Since Lemma 6 is sufficient to show the permutations through nagative rules, here we particularly show the cases for positive rules. 
1. First let us show the cases for the zero-premise rules. $\perp L$ and $T R$ are trivial, as they are applicable for an arbitrary label. The permutation for $i d$ runs as follows, where $\mathcal{G}$ is the set of relational atoms in $(\epsilon, y \triangleright x) ; \Gamma$.

$$
\frac{\mathcal{G}[x / y] \vdash_{E}\left(w_{1}[x / y]=w_{2}[x / y]\right)}{(\epsilon, x \triangleright x) ; \Gamma[x / y] ; w_{1}[x / y]: P \vdash w_{2}[x / y]: P ; \Delta}{ }^{i d}{ }^{i d}
$$

By Lemma 18, if $\mathcal{G}[x / y] \vdash_{E}\left(w_{1}[x / y]=w_{2}[x / y]\right)$ then $\mathcal{G} \vdash_{E}\left(w_{1}=w_{2}\right)$ (note that this is because $(\epsilon, y \triangleright x) \in \mathcal{G})$. Therefore we can apply $i d$ directly on the bottom sequent, and eliminate the $E q_{1}$ application.

The case for $T^{*} R$ is treated similarly. As we have shown, structural rules can permute through $\top^{*} L, \wedge L$, $\wedge R, \rightarrow L, \rightarrow R, * L$ and $-* R$, so these cases are left out here.

2. Permute $E q_{1}$ through $E$, assuming the label being replaced is $y$. The original derivation is as follows.

$$
\begin{aligned}
& \text { II } \\
& \frac{(w, x, \triangleright z) ;(x, w \triangleright z) ;(\epsilon, w \triangleright w) ; \Gamma[w / y] \vdash \Delta[w / y]}{\frac{(x, w \triangleright z) ;(\epsilon, w \triangleright w) ; \Gamma[w / y] \vdash \Delta[w / y]}{(x, y \triangleright z) ;(\epsilon, y \triangleright w) ; \Gamma \vdash \Delta} E q_{1}} E
\end{aligned}
$$

The permuted derivation is as follows.

$$
\frac{\Pi}{\frac{(w, x, \triangleright z) ;(x, w \triangleright z) ;(\epsilon, w \triangleright w) ; \Gamma[w / y] \vdash \Delta[w / y]}{\frac{(y, x \triangleright z) ;(x, y \triangleright z) ;(\epsilon, y \triangleright w) ; \Gamma \vdash \Delta}{(x, y \triangleright z) ;(\epsilon, y \triangleright w) ; \Gamma \vdash \Delta}} E q_{1}}
$$

3. Premute $E q_{1}$ through $U$, assuming the replaced label is $x$. Then the derivation runs as follows.

$$
\frac{\Pi}{\frac{(w, \epsilon \triangleright w) ;(\epsilon, w \triangleright w) ; \Gamma[w / x] \vdash \Delta[w / x]}{(\epsilon, w \triangleright w) ; \Gamma[w / x] \vdash \Delta[w / x]}} E q_{1}
$$

We modify the derivation as follows.

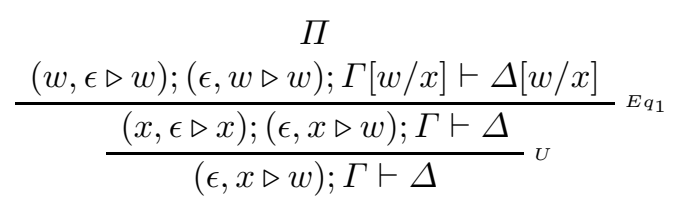

Note that we can also generate $(w, \epsilon \triangleright w)$ directly using the $U$ rule, but the effect is the same.

4. Permute $E q_{1}$ through $* R$. Suppose the principal relational atom of $E q_{1}$ is not the same as the one used in $* R$, let $\mathcal{G}$ be the set of relational atoms in $\left(\epsilon, w \triangleright w^{\prime}\right)\left(x, y \triangleright z^{\prime}\right) ; \Gamma$, the derivation runs as follows. Here we write $(\Gamma \vdash \Delta)[x / y]$ to mean that replace every $y$ by $x$ in the entire sequent. The equality entailment is $\mathcal{G}\left[w^{\prime} / w\right] \vdash_{E}\left(z\left[w^{\prime} / w\right]=z^{\prime}\left[w^{\prime} / w\right]\right)$ (to save space, we do not write the constraint in the derivation).

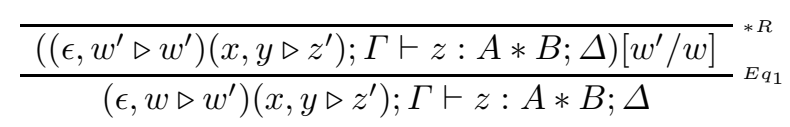

The two premises of the $* R$ rule application are listed below.

$$
\begin{gathered}
\left(\left(\epsilon, w^{\prime} \triangleright w^{\prime}\right) ;\left(; x, y \triangleright z^{\prime}\right) ; \Gamma \vdash x: A ; z: A * B ; \Delta\right)\left[w^{\prime} / w\right] \\
\left(\left(\epsilon, w^{\prime} \triangleright w^{\prime}\right) ;\left(x, y \triangleright z^{\prime}\right) ; \Gamma \vdash y: B ; z: A * B ; \Delta\right)\left[w^{\prime} / w\right]
\end{gathered}
$$

By Lemma [18, since $\mathcal{G}\left[w^{\prime} / w\right] \vdash_{E}\left(z\left[w^{\prime} / w\right]=z^{\prime}\left[w^{\prime} / w\right]\right)$, and $\left(\epsilon, w \triangleright w^{\prime}\right) \in \mathcal{G}, \mathcal{G} \vdash_{E}\left(z=z^{\prime}\right)$ holds. Therefore we have the following two derivations:

$$
\frac{\left(\left(\epsilon, w^{\prime} \triangleright w^{\prime}\right) ;\left(; x, y \triangleright z^{\prime}\right) ; \Gamma \vdash x: A ; z: A * B ; \Delta\right)\left[w^{\prime} / w\right]}{\left(\epsilon, w \triangleright w^{\prime}\right) ;\left(; x, y \triangleright z^{\prime}\right) ; \Gamma \vdash x: A ; z: A * B ; \Delta}=q_{1}
$$


and

$$
\frac{\left(\left(\epsilon, w^{\prime} \triangleright w^{\prime}\right) ;\left(x, y \triangleright z^{\prime}\right) ; \Gamma \vdash y: B ; z: A * B ; \Delta\right)\left[w^{\prime} / w\right]}{\left(\epsilon, w \triangleright w^{\prime}\right) ;\left(x, y \triangleright z^{\prime}\right) ; \Gamma \vdash y: B ; z: A * B ; \Delta} E_{1}
$$

then we use the $* R$ rule, where the equality entailment is $\mathcal{G} \vdash_{E}\left(z=z^{\prime}\right)$, to obtain the end sequent $\left(\epsilon, w \triangleright w^{\prime}\right)\left(x, y \triangleright z^{\prime}\right) ; \Gamma \vdash z: A * B ; \Delta$.

If the principal relational atom is used in the $* R$ rule, the permutation is analogous. The permutation through $-* L$ is similar.

5. Permutation through $A$. We show the case where the principal relational atom in $E q_{1}$ is not in $A$, the other cases are similar. The original derivation is as follows.

$$
\frac{\left((\epsilon, w \triangleright w) ;(u, w \triangleright z) ;(y, v \triangleright w) ;(x, y \triangleright z) ;\left(u, v \triangleright x^{\prime}\right) ; \Gamma \vdash \Delta\right)\left[w / w^{\prime}\right]}{\frac{\left((\epsilon, w \triangleright w) ;(x, y \triangleright z) ;\left(u, v \triangleright x^{\prime}\right) ; \Gamma \vdash \Delta\right)\left[w / w^{\prime}\right]}{\left(\epsilon, w^{\prime} \triangleright w\right) ;(x, y \triangleright z) ;\left(u, v \triangleright x^{\prime}\right) ; \Gamma \vdash \Delta}} E q_{1}
$$

The condition on the $A$ rule is $\mathcal{G}\left[w / w^{\prime}\right] \vdash_{E}\left(x\left[w / w^{\prime}\right]=x^{\prime}\left[w / w^{\prime}\right]\right)$. By Lemma 18, $\mathcal{G} \vdash_{E}\left(x=x^{\prime}\right)$ holds. Therefore the derivation is transformed into the following:

$$
\frac{\left(\left(\epsilon, w^{\prime} \triangleright w\right) ;(u, w \triangleright z) ;(y, v \triangleright w) ;(x, y \triangleright z) ;\left(u, v \triangleright x^{\prime}\right) ; \Gamma \vdash \Delta\right)\left[w / w^{\prime}\right]}{\frac{\left(\epsilon, w^{\prime} \triangleright w\right) ;(u, w \triangleright z) ;(y, v \triangleright w) ;(x, y \triangleright z) ;\left(u, v \triangleright x^{\prime}\right) ; \Gamma \vdash \Delta}{\left(\epsilon, w^{\prime} \triangleright w\right) ;(x, y \triangleright z) ;\left(u, v \triangleright x^{\prime}\right) ; \Gamma \vdash \Delta}}{ }_{A} q_{1}
$$

The condition on the $A$ rule is $\mathcal{G} \vdash_{E}\left(x=x^{\prime}\right)$. $A_{C}$ is treated similarly.

Lemma 20. If $(\epsilon, y \triangleright y) ; \Gamma[y / x] \vdash \Delta[y / x]$ is derivable in $L S_{B B I}^{e}$, then $(\epsilon, y \triangleright x) ; \Gamma \vdash \Delta$ is derivable in LS $S_{B B I}^{e}$.

Proof. Symmetric to the proof in Lemma 19.

Theorem 9. If a sequent is derivable in $L S_{B B I}$, then it is also derivable in $L S_{B B I}^{e}$.

Proof. Immediate by Lemma 17, 19, 20.

\section{A.10 Substitution lemma for $L S_{B B I}^{e}$}

This section proves the substitution lemma for the intermediate system $L S_{B B I}^{e}$, as this will be used in some proofs.

Lemma 21. If $\mathcal{G} \vdash_{E}(x=y)$ then for any substitution $[s / t]$, where $t \neq \epsilon, \mathcal{G}[s / t] \vdash_{E}(x[s / t]=y[s / t])$.

Proof. Let $(\mathcal{G}, \sigma, \phi)$ be the solution to $\mathcal{G} \vdash_{E}(x=y)$, we prove this lemma by induction on the length of $\sigma$.

1. Base case, $\sigma$ is an empty sequence. In this case, the sequence of substitutions $\phi$ is also empty, therefore $x=y$. As a result, it must be the case that $x[s / t]=y[s / t]$, so $\mathcal{G}[s / t] \vdash_{E}(x[s / t]=y[s / t])$ trivially holds.

2. Inductive case, assume $|\sigma|=n$. Let us look at the first rule application in $\sigma$. Assume this rule is $E q_{1}$ (the case for $E q_{2}$ is symmetric), and the principal relational atom is $(\epsilon, u \triangleright v)$, then $\sigma$ is as follows.

$$
\begin{gathered}
\mathcal{G} \phi \vdash_{E}(x \phi=y \phi) \\
\vdots \sigma^{\prime} \\
\frac{\mathcal{G}^{\prime}[v / u] ;(\epsilon, v \triangleright v) \vdash_{E}(x[v / u]=y[v / u])}{\mathcal{G}^{\prime} ;(\epsilon, u \triangleright v) \vdash_{E}(x=y)}{ }^{E q_{1}}
\end{gathered}
$$

(a) If $u=t$ and $v=s$, then the premise of the last rule application is already what we need.

(b) If $u=t$ and $v \neq s$, we obtain the desired entailment as follows $(I H[x / y]$ stands for applying the induction hypothesis with the substitution $[x / y]$, we use double line to mean that the premise and the conclusion are equivalent). 


$$
\begin{gathered}
I H[v / s] \\
\frac{\mathcal{G}^{\prime}[v / u][v / s] ;(\epsilon, v \triangleright v) \vdash_{E}(x[v / u][v / s]=y[v / u][v / s])}{\frac{\mathcal{G}^{\prime}[s / u][v / s] ;(\epsilon, v \triangleright v) \vdash_{E}(x[s / u][v / s]=y[s / u][v / s])}{\mathcal{G}^{\prime}[s / u] ;(\epsilon, s \triangleright v) \vdash_{E}(x[s / u]=y[s / u])}}
\end{gathered}
$$

(c) If $u=s$, we prove the substituted entailment as follows.

$$
I H[v / t]
$$

$$
\frac{\frac{\mathcal{G}^{\prime}[v / u][v / t] ;(\epsilon, v \triangleright v) \vdash_{E}(x[v / u][v / t]=y[v / u][v / t])}{\overline{\mathcal{G}}^{\prime}[u / t][v / u] ;(\epsilon, v \triangleright v) \vdash_{E}(x[u / t][v / u]=y[u / t][v / u])}}{\mathcal{G}^{\prime}[u / t] ;(\epsilon, u \triangleright v) \vdash_{E}(x[u / t]=y[u / t])}
$$

Note that under this case if $v=t$, the proof is just a special case of the one above.

(d) If $v=t$, the case is shown below.

(e) If $v=s$, the proof is as follows.

$$
\begin{gathered}
I H[s / v] \\
\frac{\mathcal{G}^{\prime}[v / u][s / v] ;(\epsilon, s \triangleright s) \vdash_{E}(x[v / u][s / v]=y[v / u][s / v])}{\frac{\mathcal{G}^{\prime}[s / v][s / u] ;(\epsilon, s \triangleright s) \vdash_{E}(x[s / v][s / u]=y[s / v][s / u])}{\mathcal{G}^{\prime}[s / v] ;(\epsilon, u \triangleright s) \vdash_{E}(x[s / v]=y[s / v])}}
\end{gathered}
$$

$$
\begin{gathered}
I H[v / t] \\
\frac{\mathcal{G}^{\prime}[v / u][v / t] ;(\epsilon, v \triangleright v) \vdash_{E}(x[v / u][v / t]=y[v / u][v / t])}{\frac{\mathcal{G}^{\prime}[v / t][v / u] ;(\epsilon, v \triangleright v) \vdash_{E}(x[v / t][v / u]=y[v / t][v / u])}{\mathcal{G}^{\prime}[v / t] ;(\epsilon, u \triangleright v) \vdash_{E}(x[v / t]=y[v / t])}} \\
E q_{1}
\end{gathered}
$$

(f) If $[s / t]$ and $[u / v]$ are independent, then we can switch the order of substitution, and derive the entailment as follows.

$$
\begin{gathered}
I H[s / t] \\
\frac{\mathcal{G}^{\prime}[v / u][s / t] ;(\epsilon, v \triangleright v) \vdash_{E}(x[v / u][s / t]=y[v / u][s / t])}{\frac{\mathcal{G}^{\prime}[s / t][v / u] ;(\epsilon, v \triangleright v) \vdash_{E}(x[s / t][v / u]=y[s / t][v / u])}{\mathcal{G}^{\prime}[s / t] ;(\epsilon, u \triangleright v) \vdash_{E}(x[s / t]=y[s / t])}} \\
E q_{1}
\end{gathered}
$$

Since substitution does not break the equality entailment, we can show a substitution lemma for the system $L S_{B B I}^{e}$.

Lemma 22 (Substitution in $L S_{B B I}^{e}$ ). If there is a derivation for the sequent $\Gamma \vdash \Delta$ in $L S_{B B I}^{e}$ then there is a derivation of the same height for the sequent $\Gamma[y / x] \vdash \Delta[y / x]$ in $L S_{B B I}^{e}$, where every occurrence of label $x(x \neq \epsilon)$ is replaced by label $y$.

Proof. The proof is basically the same as the one for $L S_{B B I}$, since there are a lot of common rules. For the rules that are changed, the case for $T^{*} L$ is similar to those cases for additive rules. The proof for the rest of changed rules are straightforward with the help of Lemma 21.

\section{A.11 Soundness of $L S_{B B I}^{s f}$}

Theorem 10. If there is a derivation $\Pi$ for a sequent $\mathcal{G} \| \Gamma \vdash \Delta$ in $L S_{B B I}^{s f}$, then there is a derivation $\Pi^{\prime}$ for the sequent $\mathcal{G} ; \Gamma \vdash \Delta$ in $L S_{B B I}^{e}$.

Proof. The soundness proof for this system is rather straightforward. To prove this, we show that each rule in $L S_{B B I}^{s f}$ can be simulated in $L S_{B B I}^{e}$. To do this, one just need to unfold the structural rule applications into the derivation. For instance, we can simulate the $i d$ rule in $L S_{B B I}^{s f}$ by using the following rules in $L S_{B B I}^{e}$ :

$$
\begin{gathered}
\frac{\mathcal{S}(\mathcal{G}, \sigma) \vdash_{E}\left(w_{1}=w_{2}\right)}{\mathcal{S}(\mathcal{G}, \sigma) ; \Gamma ; w_{1}: P \vdash w_{2}: P ; \Delta}{ }^{i d} \\
\vdots \sigma \\
\mathcal{G} ; \Gamma ; w_{1}: P \vdash w_{2}: P ; \Delta
\end{gathered}
$$


The above works because the $i d$ rule in $L S_{B B I}^{s f}$ requires $\mathcal{G} \vdash_{R}\left(w_{1}=w_{2}\right)$, which by definition ensures that $\mathcal{S}(\mathcal{G}, \sigma) \vdash_{E}\left(w_{1}=w_{2}\right)$ holds. The case for $T^{*} R$ works similarly. One thing to notice is that structural rules only add relational atoms into the current set, so except for $\mathcal{G}$ is becoming a bigger set, all the other structures in the sequent remain the same after the sequence $\sigma$ of applications. Let us examine the simulation of $* R$ in $L S_{B B I}^{e}$.

$$
\begin{gathered}
\mathcal{S}(\mathcal{G}, \sigma) ; \Gamma \vdash x^{\prime}: A ; w: A * B ; \Delta \quad \mathcal{S}(\mathcal{G}, \sigma) ; \Gamma \vdash y^{\prime}: B ; w: A * B ; \Delta \\
\mathcal{S}(\mathcal{G}, \sigma) ; \Gamma \vdash w: A * B ; \Delta \\
\vdots \sigma \\
\mathcal{G} ; \Gamma \vdash w: A * B ; \Delta
\end{gathered}
$$

The condition of the $* R$ rule is $\mathcal{S}(\mathcal{G}, \sigma) \vdash_{E}\left(w=w^{\prime}\right)$. Since the $L S_{B B I}^{s f}$ rule requires $\mathcal{G} \vdash_{R}(x, y \triangleright w)$, which by definition ensures that there is a solution $(\mathcal{G}, \sigma)$ such that $\left(x^{\prime}, y^{\prime} \triangleright w^{\prime}\right) \in \mathcal{S}(\mathcal{G}, \sigma)$, and the following holds:

$$
\begin{aligned}
& \mathcal{S}(\mathcal{G}, \sigma) \vdash_{E}\left(x=x^{\prime}\right) \\
& \mathcal{S}(\mathcal{G}, \sigma) \vdash_{E}\left(y=y^{\prime}\right) \\
& \mathcal{S}(\mathcal{G}, \sigma) \vdash_{E}\left(w=w^{\prime}\right)
\end{aligned}
$$

The last relation entailment is enough to guarantee that the $* R$ rule is applicable. To restore each branch, we need the Lemma 22 (Substitution lemma for $L S_{B B I}^{e}$ ). Here we use double line to indicate the premise and the conclusion are equivalent. Let us look at the left branch. By the first relation entailment, there is a sequence $\sigma^{\prime}$ of $E q_{1}, E q_{2}$ applications so that $x \theta=x^{\prime} \theta$. Therefore we can construct a proof for the left branch as follows.

$$
\begin{gathered}
\mathcal{S}(\mathcal{G}, \sigma) ; \Gamma \vdash x: A ; w: A * B ; \Delta \\
\overline{\mathcal{S}(\overline{\mathcal{G}}, \bar{\sigma}) \bar{\theta} ; \bar{\Gamma}-\bar{\vdash}-\bar{\theta}: \bar{A} ; w \theta: \bar{A} * \bar{B} ; \bar{\Delta} \theta} \\
\hline \hline \mathcal{S}(\mathcal{G}, \sigma) \theta ; \Gamma \theta \vdash x^{\prime} \theta: A ; w \theta: A * B ; \Delta \theta \\
\vdots \sigma^{\prime} \\
\mathcal{S}(\mathcal{G}, \sigma) ; \Gamma \vdash x^{\prime}: A ; w: A * B ; \Delta
\end{gathered}
$$

The case for $-* L$ is analogous. The rest rules are the same as in $L S_{B B I}^{e}$, thus we conclude that the rules in $L S_{B B I}^{s f}$ are sound.

\section{A.12 Completeness of $L S_{B B I}^{s f}$}

The completeness proof runs the same as in $L S_{B B I}^{e}$ : if we add the structural rules $E, U, A, A_{C}$ in $L S_{B B I}^{s f}$, then it becomes a superset of $L S_{B B I}^{e}$. Then we prove that these rules are admissible in $L S_{B B I}^{s f}$ by showing they can permute through $* R,-* L, i d$, and $T^{*} R$.

First of all, let us show that when we add $E, U, A, A_{C}$ (from $L S_{B B I}^{e}$ ) to $L S_{B B I}^{s f}$, its rules can simulate those ones in $L S_{B B I}^{e}$. As most of the rules are identical, the key part is the show the relation entailment is as powerful as the equality entailment. This is "built-in" the definition, so there is no surprise.

Lemma 23. If $\mathcal{G} \vdash_{E}\left(w_{1}=w_{2}\right)$, then $\mathcal{G} \vdash_{R}\left(w_{1}=w_{2}\right)$.

Proof. Let $\sigma$ be an empty list of rule applications, then $\mathcal{S}(\mathcal{G}, \emptyset)=\mathcal{G}$. Therefore by definition $\mathcal{G} \vdash_{R}\left(w_{1}=w_{2}\right)$.

If we change $\vdash_{R}$ to $\vdash_{E}$ in $L S_{B B I}^{s f}$, every rule is the same as the one in $L S_{B B I}^{e}$. Therefore $L S_{B B I}^{s f}+E+$ $U+A+A_{C}$ is at least as powerful as $L S_{B B I}^{e}$.

Lemma 24. The rules $E, U, A$, and $A_{C}$ are admissible in $L S_{B B I}^{s f}$.

Proof. We show that the said rules can permute upwards through $i d, \top^{*} R, * R$ and $-* L$, the other cases are cover by Lemma 6. We only give some examples here, the others are similar. The heart of the argument is that the application of structural rules are hidden inside the relation entailment, so we do not have to apply them explicitly.

Permute $E$ through $i d$, the suppose the original derivation runs as follows. 


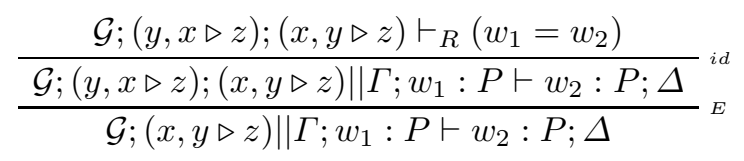

The permuted derivation is:

$$
\frac{\mathcal{G} ;(x, y \triangleright z) \vdash_{R}\left(w_{1}=w_{2}\right)}{\mathcal{G} ;(x, y \triangleright z) \| \Gamma ; w_{1}: P \vdash w_{2}: P ; \Delta} i d
$$

Assume $\mathcal{G} ;(y, x \triangleright z) ;(x, y \triangleright z) \vdash_{R}\left(w_{1}=w_{2}\right)$ is derived by applying a sequence $\sigma$ of structural rules. Then $\mathcal{S}\left((\mathcal{G} ;(x, y \triangleright z)), \sigma^{\prime}\right)$ can prove $\mathcal{G} ;(x, y \triangleright z) \vdash_{R}\left(w_{1}=w_{2}\right)$, where $\sigma^{\prime}$ is $E(\{(x, y \triangleright z)\}, \emptyset)$ followed by $\sigma$. That is, the application of $E$ is absorbed in $\vdash_{R}$.

Permute $A$ through $i d$, the argument is similar. The original derivation is:

$$
\frac{\frac{\mathcal{G} ;(u, w \triangleright z) ;(y, v \triangleright w) ;(x, y \triangleright z) ;\left(u, v \triangleright x^{\prime}\right) \vdash_{R}\left(w_{1}=w_{2}\right)}{\mathcal{G} ;(u, w \triangleright z) ;(y, v \triangleright w) ;(x, y \triangleright z) ;\left(u, v \triangleright x^{\prime}\right)|| \Gamma ; w_{1}: P \vdash w_{2}: P ; \Delta}}{\mathcal{G} ;(x, y \triangleright z) ;\left(u, v \triangleright x^{\prime}\right)|| \Gamma ; w_{1}: P \vdash w_{2}: P ; \Delta}{ }_{A}
$$

The condition on the rule $A$ is $\mathcal{G} ;(x, y \triangleright z) ;\left(u, v \triangleright x^{\prime}\right) \vdash_{E}\left(x=x^{\prime}\right)$. Then we can omit the application of $A$, since $\mathcal{G} ;(u, w \triangleright z) ;(y, v \triangleright w) ;(x, y \triangleright z) ;\left(u, v \triangleright x^{\prime}\right) \vdash_{R}\left(w_{1}=w_{2}\right)$ implies $\mathcal{G} ;(x, y \triangleright z) ;\left(u, v \triangleright x^{\prime}\right) \vdash_{R}\left(w_{1}=w_{2}\right)$, one just need to add the $A$ application ahead to the sequence of structural rules that derives the former relation entailment to get a new sequence of rules to derive the latter one.

\section{A.13 Soundness of $F V L S_{B B I}$}

Proof of Theorem 6 .

Proof. By induction on the height $n$ of derivation $\Pi$.

1. Base case: $n=1$. In this case, we can only use a zero-premise rule to prove the sequent. Since the sequent is ground, there are no free variables. Thus the constraint generated by the rule application is a simple constraint, of the form $\mathcal{G} \vdash ?_{R}^{?}(a=b)$ or $\mathcal{G} \vdash{ }_{R}^{?}(a=\epsilon)$. A solution of this constraint is simply a derivation $\sigma$ of $\mathcal{G} \vdash_{R}(a=b)$ (resp. $\mathcal{G} \vdash(a=\epsilon)$. In either case, this translates straightforwardly into a derivation in $L S_{B B I}^{s f}$ with the same rule.

2. Inductive case: $n>1$. This can be done by a case analysis of the last rule application in $\Pi$. We demonstrate the case for $* R$, where a constraint is generated. The case for $-* L$ is analogous, and the other cases are easy since we can use the induction hypothesis directly. Suppose $\Pi$ runs as follows.

$$
\begin{aligned}
& \Pi_{1} \quad \Pi_{2} \\
& \frac{\mathcal{G}\|\Gamma \vdash \mathbf{x}: A ; w: A * B ; \Delta \quad \mathcal{G}\| \Gamma \vdash \mathbf{y}: B ; w: A * B ; \Delta}{\mathcal{G} \| \Gamma \vdash w: A * B ; \Delta} * R
\end{aligned}
$$

Suppose $\mathbb{C}(\Pi)=\left(\left\{\mathfrak{c}_{1}, \ldots, \mathfrak{c}_{k}\right\}, \preceq\right)$, for some $k \geq 1$. Suppose that the constraint generated by this rule application is $\mathcal{G} \vdash_{R}^{?}(\mathbf{x}, \mathbf{y} \triangleright w)$ and it corresponds to $\mathfrak{c}_{i}$ for some $i \in\{1, \ldots, k\}$. By the assumption, there is a solution $\left(\theta,\left\{\sigma_{1}, \cdots, \sigma_{k}\right\}\right)$ for the constraint system $\mathbb{C}=\left(\mathcal{C}(\Pi), \preceq^{\Pi}\right)$. Now $\mathfrak{c}_{i}$ must be a simple constraint in $\mathbb{C}$, as the end sequent is ground. Let $\left(\theta_{i}, \sigma_{i}\right)$ be the solution to $\mathfrak{c}_{i}$, where $\theta_{i}$ is a restriction to $\theta$ containing $\mathbf{x}$ and $\mathbf{y}$, and $\sigma_{i} \in\left\{\sigma_{1}, \cdots, \sigma_{k}\right\}$. By definition of the solution to a simple constraint, $\sigma_{i}$ is a derivation of $\mathcal{G} \vdash_{R}\left(\mathbf{x} \theta_{i}, \mathbf{y} \theta_{i} \triangleright w\right)$. Therefore in $L S_{B B I}^{s f}$, to derive the end sequent, we apply $* R$ backwards:

$$
\frac{\mathcal{S}\left(\mathcal{G}, \sigma_{i}\right)\left\|\Gamma \vdash \mathbf{x} \theta_{i}: A ; w: A * B ; \Delta \quad \mathcal{S}\left(\mathcal{G}, \sigma_{i}\right)\right\| \Gamma \vdash \mathbf{y} \theta_{i}: B ; w: A * B ; \Delta}{\mathcal{G} \| \Gamma \vdash w: A * B ; \Delta} * R
$$

The condition on this rule is $\mathcal{G} \vdash_{R}\left(\mathbf{x} \theta_{i}, \mathbf{y} \theta_{i} \triangleright w\right)$. Now we construct the derivation for both branches in the following way. Firstly we substitute $\mathbf{x}$ and $\mathbf{y}$ with $\mathbf{x} \theta_{i}$ and $\mathbf{y} \theta_{i}$ respectively in $\Pi_{1}$ and $\Pi_{2}$, making the end sequents in the two derivations ground. Let us refer to the modified derivations as $\Pi_{1}^{\prime}$ and $\Pi_{2}^{\prime}$ respectively. Then for each sequent in $\Pi_{1}^{\prime}$ and $\Pi_{2}^{\prime}$ and each constraint in $\mathcal{C}\left(\Pi_{1}^{\prime}\right) \cup \mathcal{C}\left(\Pi_{2}^{\prime}\right)$, we change the set of relational atoms to be the union of $\mathcal{S}\left(\mathcal{G}, \sigma_{i}\right)$ and the original one. This is harmless because we 
can use weakening to obtain the same sequents as in $\Pi_{1}^{\prime}$ and $\Pi_{2}^{\prime}$, and weakening is height-preserving admissible. Let the resultant derivations be $\Pi_{1}^{\prime \prime}$ and $\Pi_{2}^{\prime \prime}$ respectively. Now the end sequents of $\Pi_{1}^{\prime \prime}$ and $\Pi_{2}^{\prime \prime}$ are respectively just the same as the two branches we created in the $L S_{B B I}^{s f}$ derivation. Moreover, each constraint in $\mathcal{C}\left(\Pi_{1}^{\prime \prime}\right) \cup \mathcal{C}\left(\Pi_{2}^{\prime \prime}\right)$ is in the restricted constraint system $\mathbb{C}^{\prime}=\left(\mathcal{C}(\Pi), \preceq^{\Pi}\right) \uparrow\left(\mathfrak{c}_{i}, \theta_{i}, \sigma_{i}\right)$, which has a solution $\left(\theta \backslash \theta_{i},\left\{\sigma_{1}, \cdots, \sigma_{k}\right\} \backslash \sigma_{i}\right)$, and obeys the partial order $\preceq^{\prime}$. Further, as $\Pi_{1}^{\prime \prime}$ (resp. $\Pi_{2}^{\prime \prime}$ ) uses the same rule applications as in $\Pi_{1}$ (resp. $\left.\Pi_{2}\right)$, the order of constraints is preserved. That is, in the constraints system $\mathbb{C}_{1}=\left(\mathcal{C}\left(\Pi_{1}^{\prime \prime}\right), \preceq \Pi_{1}^{\prime \prime}\right)\left(\right.$ resp. $\left.\mathbb{C}_{2}=\left(\mathcal{C}\left(\Pi_{2}^{\prime \prime}\right), \preceq \Pi_{2}^{\prime \prime}\right)\right)$, if $\mathfrak{c} \preceq \Pi_{1}^{\prime \prime} \mathfrak{c}^{\prime}\left(\right.$ resp. $\left.\mathfrak{c} \preceq \Pi_{2}^{\prime \prime} \mathfrak{c}^{\prime}\right)$ then $\mathfrak{c} \preceq^{\prime} \mathfrak{c}^{\prime}$ in $\mathbb{C}^{\prime}$. Therefore we can construct the solution $\left(\theta_{1}^{\prime \prime}, \Sigma_{1}\right)$ to $\mathbb{C}_{1}$ (and analogously to $\mathbb{C}_{2}$ ) as follows.

$$
\begin{aligned}
\theta_{1}^{\prime \prime} & =\left(\theta \backslash \theta_{i}\right) \uparrow f v\left(\mathcal{C}\left(\Pi_{1}^{\prime \prime}\right)\right) \\
\Sigma_{1} & =\left\{\sigma \mid \mathfrak{c} \in \mathcal{C}\left(\Pi_{1}^{\prime \prime}\right), \sigma \in\left\{\sigma_{1}, \cdots, \sigma_{k}\right\} \backslash \sigma_{i}, \text { and } \sigma=\operatorname{dev}(\mathfrak{c})\right\}
\end{aligned}
$$

By the induction hypothesis, we can obtain a $L S_{B B I}^{s f}$ derivation for each branch.

\section{A.14 Completeness of $F V L S_{B B I}$}

Proof of Theorem 7

Proof. We describe the construction from a $L S_{B B I}^{s f}$ derivation $\Pi$ to a $F V L S_{B B I}$ derivation $\Pi^{\prime}$. We need to prove a stronger invariant: for each sequent $\mathcal{G}_{E} ; \mathcal{G}_{S} \| \Gamma \vdash \Delta$ in $\Pi$, if there exists a triple consisting of:

- a symbolic sequent $\mathcal{G}_{E}^{\prime} \| \Gamma^{\prime} \vdash \Delta^{\prime}$,

- a well-formed constraint system $\mathbb{C}=(\mathcal{C}, \preceq)$,

- and a solution $S=(\theta,\{\vec{\sigma}\})$ to $\mathbb{C}$

such that

- $X$ is a thread of $\mathbb{C}$ consisted of $f v\left(\mathcal{G}_{E}^{\prime} \| \Gamma^{\prime} \vdash \Delta^{\prime}\right)$,

$-\mathcal{G}_{E}^{\prime} \theta=\mathcal{G}_{E}, \Gamma^{\prime} \theta=\Gamma, \Delta^{\prime} \theta=\Delta$ and

$-\mathcal{G}_{E} \cup \mathcal{G}_{S}=\mathcal{S}^{*}(\mathbb{C}, S, X)$

then there is a symbolic derivation $\Psi$ of $\mathcal{G}_{E}^{\prime} \| \Gamma^{\prime} \vdash \Delta^{\prime}$ such that $\mathbb{C}{ }^{\circ} X \mathbb{C}(\Psi)$ is well-formed and solvable.

First of all, by Lemma 10, since the end sequent in $\Psi$ only contains the free variables occur in $X$, the composition $\mathbb{C} \circ^{X} \mathbb{C}(\Psi)$ must be well-formed. Thus we only need to show that there is a solution to this constraint system. We prove this by case analysis on the last rule in $\Pi$, and show that in each case, for each premise of the rule, one can find a triple satisfying the above property, such that the symbolic sequent(s) in the premise(s), together with the one in the conclusion form a valid inference in $F V L S_{B B I}$. We illustrate it here with a case when $\Pi$ ends with $* R$ :

Suppose $\Pi$ ends with $* R$, where the conclusion, the premises, and the relational entailment are respectively:

$-\mathcal{G}_{E} ; \mathcal{G}_{S} \| \Gamma \vdash w: A * B ; \Delta$

$-\mathcal{S}\left(\left(\mathcal{G}_{E} ; \mathcal{G}_{S}\right), \sigma\right) \| \Gamma \vdash w_{1}: A ; w: A * B ; \Delta$

$-\mathcal{S}\left(\left(\mathcal{G}_{E} ; \mathcal{G}_{S}\right), \sigma\right) \| \Gamma \vdash w_{2}: B ; w: A * B ; \Delta$

$-\mathcal{G}_{E} ; \mathcal{G}_{S} \vdash_{R}\left(w_{1}, w_{2} \triangleright w\right)$

and suppose that the relation in the last item is derived via $\sigma$. Suppose that we can find a triple consisting of

- a symbolic sequent $\mathcal{G}_{E}^{\prime} \| \Gamma^{\prime} \vdash \mathbf{w}: A * B ; \Delta^{\prime}$

- a well-formed constraint system $\mathbb{C}=(\mathcal{C}, \preceq)$, and

- a solution $S=\left(\theta,\left\{\sigma_{1}, \ldots, \sigma_{n}\right\}\right)$ to $\mathbb{C}$

satisfying the following:

- $X$ is a thread of $\mathbb{C}$ consisted of $f v\left(\mathcal{G}_{E}^{\prime} \| \Gamma^{\prime} \vdash \mathbf{w}: A * B ; \Delta^{\prime}\right)$,

$-\mathcal{G}_{E}^{\prime} \theta=\mathcal{G}_{E}, \Gamma^{\prime} \theta=\Gamma, \Delta^{\prime} \theta=\Delta, w=\mathbf{w} \theta$ and

$-\mathcal{G}_{E} \cup \mathcal{G}_{S}=\mathcal{S}^{*}(\mathbb{C}, S, X)$. 
We need to show that we can find such triples for the premises, and more importantly, the symbolic sequents in the premises are related to the symbolic sequent in the conclusion via $* R$. In this case, the symbolic sequents are simply the following:

1. $\mathcal{G}_{E}^{\prime} \| \Gamma^{\prime} \vdash \mathbf{x}: A ; \mathbf{w}: A * B ; \Delta^{\prime}$, for the left premise,

2. $\mathcal{G}_{E}^{\prime} \| \Gamma^{\prime} \vdash \mathbf{y}: A ; \mathbf{w}: A * B ; \Delta^{\prime}$, for the right premise.

The constraint systems are: $\mathbb{C}^{\prime}=\left(\mathcal{C} \cup\left\{\mathfrak{c}_{j}\right\}, \preceq^{\prime}\right)$ for both premises, where $\mathfrak{c}_{j}=\mathcal{G}_{E}^{\prime} \vdash_{R}^{?}(\mathbf{x}, \mathbf{y} \triangleright \mathbf{w})$ and $\preceq^{\prime}$ is $\preceq$ extended with $\mathfrak{c}(\operatorname{end}(X)) \preceq^{\prime} \mathfrak{c}_{j}$. The solutions, for both premises, are the tuple $S^{\prime}=\left(\theta^{\prime}, \Sigma\right)$ where $\theta^{\prime}=\theta \cup\left\{\mathbf{x} \mapsto w_{1}, \mathbf{y} \mapsto w_{2}\right\}$ and $\Sigma=\left\{\sigma_{1}, \ldots, \sigma_{n}, \sigma\right\}$. It is guaranteed that $\theta^{\prime}$ is enough to make both premises grounded, as $\mathbf{x}$ and $\mathbf{y}$ are the only two new free variables. The threads of free variables $X_{1}$ and $X_{2}$ for the two premises are naturally $X @[\mathbf{x}]$ and $X @[\mathbf{y}]$ respectively. By Proposition 1 , in each premise, the following holds:

$$
\mathcal{G}_{E} \cup \mathcal{G}_{S}^{\prime}=\mathcal{S}\left(\mathcal{G}_{E} \cup \mathcal{G}_{S}, \sigma\right)=\mathcal{S}\left(\mathcal{G}_{E} \cup \mathcal{G}_{S} \cup \mathcal{G}_{E}^{\prime} \theta, \sigma\right)=\mathcal{S}^{*}\left(\mathbb{C}^{\prime}, S^{\prime}, X_{1}\right)=\mathcal{S}^{*}\left(\mathbb{C}^{\prime}, S^{\prime}, X_{2}\right) .
$$

So by the induction hypothesis we have a symbolic derivation $\Pi_{1}^{\prime}$ for sequent (1) and a symbolic derivation $\Pi_{2}^{\prime}$ for sequent $(2)$, such that $\mathbb{C}_{\beta 1}=\mathbb{C}^{\prime} \circ^{X_{1}} \mathbb{C}\left(\Pi_{1}^{\prime}\right)$ and $\mathbb{C}_{\beta 2}=\mathbb{C}^{\prime} \circ^{X_{2}} \mathbb{C}\left(\Pi_{2}^{\prime}\right)$ are both solvable. Suppose the solutions are respectively $\left(\theta^{\prime} \cup \theta_{1}, \Sigma \cup \Sigma_{1}\right)$ and $\left(\theta^{\prime} \cup \theta_{2}, \Sigma \cup \Sigma_{2}\right)$. Then construct $\Pi^{\prime}$ by applying the $* R$ rule to $\Pi_{1}^{\prime}$ and $\Pi_{2}^{\prime}$. Note that the variables created in $\Pi_{1}^{\prime}$ are $\Pi_{2}^{\prime}$ are distinct so their constraints are independent of each other. So we can construct $\mathbb{C}_{p}=\mathbb{C}\left(\Pi_{1}^{\prime}\right) \circ^{\emptyset} \mathbb{C}\left(\Pi_{2}^{\prime}\right)=\left(\mathcal{C}_{p}, \preceq_{p}\right)$, along an empty thread $\emptyset$. Now $\mathbb{C}\left(\Pi^{\prime}\right)$ is obtained as $\left(\mathcal{C}_{p} \cup\left\{\mathfrak{c}_{j}\right\}, \preceq^{\Pi^{\prime}}\right)$, where $\preceq^{\Pi^{\prime}}$ is derived as follows.

- If $\mathfrak{c} \preceq_{p} \mathfrak{c}^{\prime}$ in $\mathbb{C}_{p}$, then $\mathfrak{c} \preceq^{\Pi^{\prime}} \mathfrak{c}^{\prime}$ in $\mathbb{C}\left(\Pi^{\prime}\right)$

- For any minimum constraint $\mathfrak{c}_{m}$ in $\mathbb{C}_{p}, \mathfrak{c}_{j} \preceq^{\Pi^{\prime}} \mathfrak{c}_{m}$ in $\mathbb{C}\left(\Pi^{\prime}\right)$

The solution to $\mathbb{C}_{\alpha}=\mathbb{C} \circ^{X} \mathbb{C}\left(\Pi^{\prime}\right)$ is constructed as the combination of the solutions to $\mathbb{C}_{\beta 1}$ and $\mathbb{C}_{\beta 2}$ : $\left(\theta^{\prime} \cup \theta_{1} \cup \theta_{2}, \Sigma \cup \Sigma_{1} \cup \Sigma_{2}\right)$. This construction of the solution is indeed valid, because the symbolic derivation that gives $\mathbb{C}_{\alpha}$ also yields exactly $\mathbb{C}_{\beta 1}$ and $\mathbb{C}_{\beta 2}$ (respectively on its two branches created by the $* R$ rule).

\section{A.15 The Proof of the Heuristic Method}

In the following proofs we use the tree representation of a set of relational atoms. Given a labelled binary tree $t r$ as defined in Section [6, we say another labelled binary tree $t r^{\prime}$ is a permutation of $t r$ if they have the same root and same multiset of leaves. A permutation on $t r$ is generally done by applying the rules $E, A$ on $\operatorname{Rel}(t r)$. Figure 11 gives some examples on tree permutations. In Figure 11, (b) is permuted from (a) by using $E$ on $(d, e \triangleright b)$, whereas (c) is permuted from (a) by using $A$ on the two relational atoms in the original tree.

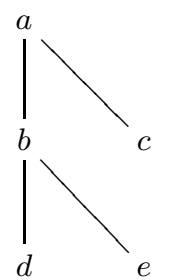

(a)

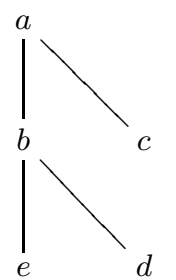

(b)

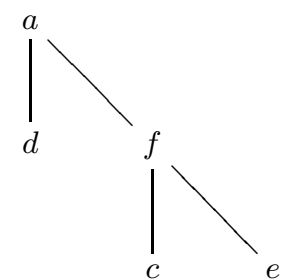

(c)

Fig. 11. Examples of tree permutations.

Lemma 25. Let $t r$ be a labelled binary tree with a root labelled with $r$ and a multiset of labels $L$ for the leaves. If there is a labelled binary tree $t^{\prime}$ with the same root and leaves labels respectively, then there is a variant $t r^{\prime \prime}$ of $t r^{\prime}$ and a sequence $\sigma$ of $E, A$ rule applications such that $\operatorname{Rel}\left(\operatorname{tr}^{\prime \prime}\right) \subseteq \mathcal{S}(\operatorname{Rel}(t r), \sigma)$. 
Proof. Prove by induction on the width of the tree $t r$. We show that any distinct permutation(i.e., they are not variants of each other) of a tree can be achieved by using the rules $E$ and $A$. Base case is when there are only two leaves in $t r$. In this case, there is only one relational atom in $\operatorname{Rel}(t r)$, thus clearly there is only one distinct permutation of $t r$, which can be obtained by applying $E$ on $\operatorname{Rel}(t r)$.

The next case is when there are 3 leaves in the tree, meaning $\operatorname{Rel}(t r)$ contains two relational atoms. In this case, it can be easily checked that there are 12 distinct permutations of $t r$, all of which can be derived by using $E$ and $A$.

Inductive case, suppose the lemma holds for all trees with width less than $n$, consider a tree $t r$ with width $n$. Suppose further that the root label of $t r$ is $r$, it's two children are in the relational atom $\left(w_{1}, w_{2} \triangleright r\right)$, and the multisets of leaves labels for the subtrees of $w_{1}$ and $w_{2}$ are $L_{1}, L_{2}$ respectively. Let $t r^{\prime}$ be a permutation of $t r$ with the same root label and leaves labels, and in $t r^{\prime}$ the two children of the root label are in the relational atom $\left(w_{3}, w_{4} \triangleright r\right)$. Suppose the multisets of leave labels for the subtrees of $w_{3}, w_{4}$ are $L_{3}, L_{4}$ respectively. Apparently, since $L_{1} \cup L_{2}=L_{3} \cup L_{4}=L$, every label in $L_{3}$ is either in $L_{1}$ or in $L_{2}$. Let $L^{\prime}=L_{1} \cap L_{3}$ and $L^{\prime \prime}=L_{2} \cap L_{3}$, then $L^{\prime} \cup L^{\prime \prime}=L_{3}$ and $\left(L_{1} \backslash L^{\prime}\right) \cup\left(L_{2} \backslash L^{\prime \prime}\right)=L_{4}$. By the induction hypothesis on the subtrees of $w_{1}$ and $w_{2}$, there exist $w_{5}, w_{6}, w_{7}, w_{8}$ s.t. $\left(w_{5}, w_{6} \triangleright w_{1}\right),\left(w_{7}, w_{8} \triangleright w_{2}\right)$ hold, and the subtrees of $w_{5}, w_{6}, w_{7}, w_{8}$ give the multisets of leaves $L^{\prime},\left(L_{1} \backslash L^{\prime}\right), L^{\prime \prime},\left(L_{2} \backslash L^{\prime \prime}\right)$ respectively. Then we use the following derivation to permute the tree:

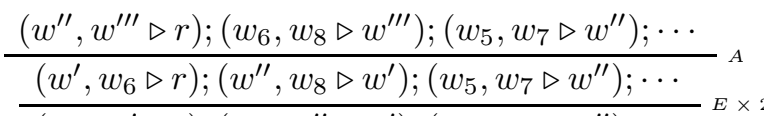

$$
\begin{aligned}
& \frac{\left(w_{6}, w^{\prime} \triangleright r\right) ;\left(w_{8}, w^{\prime \prime} \triangleright w^{\prime}\right) ;\left(w_{5}, w_{7} \triangleright w^{\prime \prime}\right) ; \cdots}{\left(w_{6}, w^{\prime} \triangleright r\right) ;\left(w_{2}, w_{5} \triangleright w^{\prime}\right) ;\left(w_{8}, w_{7} \triangleright w_{2}\right) ; \cdots}{ }_{E}^{A}
\end{aligned}
$$

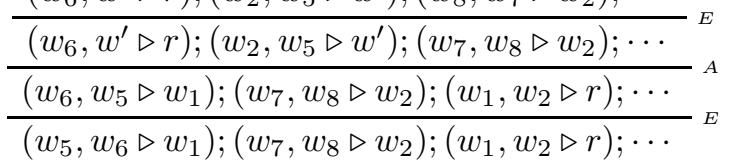

Now the subtrees of $w^{\prime \prime}$ and $w^{\prime \prime \prime}$ has the same multisets of leaves as $w_{3}$ and $w_{4}$ respectively. Again by the induction hypothesis on the subtrees of $w^{\prime \prime}$ and $w^{\prime \prime \prime}$, we obtain a tree $t r^{\prime \prime}$ which is a variant of $t r^{\prime}$.

\section{Proof of Lemma 11.}

Proof. The lemma restricts the labels of internal nodes to be free variables that are created after all the labels on the left hand side. Additionally, each free variable is only allowed to occur once in a tree. Therefore given a set $\mathcal{G}$ of relational atoms as the left hand side of those constraints, and any sequence $\sigma$ of structural rule applications, the free variable labels for internal nodes can be assigned to any labels occur in $\mathcal{S}(\mathcal{G}, \sigma)$. By Lemma 25. there exists a sequence $\sigma$ of $E, A$ applications which converts the tree on the left hand side to a tree which is a variant of the one on the right hand side, thus those constraints can be solved by assigning the free variables in the internal nodes to the corresponding labels.

\section{A.16 Proof of Formulae in the Conclusion}

In this section we show the proofs of the four formulae in the conclusion. We extend $L S_{B B I}$ in the obvious way to handle the additive connectives $\neg$ and $\vee$, where $\neg p=p \rightarrow \perp$ and $p \vee q=\neg(\neg p \wedge \neg q)$. Thus we obtain the left and right rules for $\neg, \vee$ as in the classical setting. To save space, we shall write $r^{n}$ to mean the rule $r$ is applied $n$ times, and write $r_{1} ; r_{2}$ to mean apply $r_{1}$ then apply $r_{2}$ on a sequent, when the order of rule applications does not matter.

1. To prove the formula $(F * F) \rightarrow F$, where $F=\neg\left(\top-* \neg \top^{*}\right)$, we use the following derivation in $L S_{B B I}$ : 


$$
\begin{gathered}
\frac{\left(w^{\prime}, w^{\prime \prime} \triangleright \epsilon\right) ;\left(b^{\prime}, c^{\prime} \triangleright w^{\prime \prime}\right) ;\left(b, c \triangleright w^{\prime}\right) ;(b, c \triangleright a) ; \cdots}{\left(w^{\prime}, c^{\prime} \triangleright w\right) ;\left(w, b^{\prime} \triangleright \epsilon\right) ; \cdots} A \\
\frac{\frac{\left(c^{\prime}, w^{\prime} \triangleright w\right) ;\left(b, c \triangleright w^{\prime}\right) ;\left(b^{\prime}, w \triangleright \epsilon\right) ; \cdots}{\left(b^{\prime}, w \triangleright \epsilon\right) ;(\epsilon, b \triangleright w) ;\left(c^{\prime}, c \triangleright \epsilon\right) ; \cdots}}{\frac{(b, c \triangleright a) ;\left(b^{\prime}, b \triangleright \epsilon\right) ;\left(c^{\prime}, c \triangleright \epsilon\right) ;(\epsilon, \epsilon \triangleright \epsilon) ; \cdots}{2}} A \\
\frac{\frac{(b, c \triangleright a) ;\left(b^{\prime}, b \triangleright \epsilon\right) ;\left(c^{\prime}, c \triangleright \epsilon\right) ; a: \top-* \neg \top^{*} ; b^{\prime}: \top, c^{\prime}: \top \vdash}{(b, c \triangleright a) ;\left(b^{\prime}, b \triangleright b^{\prime \prime}\right) ;\left(c^{\prime}, c \triangleright c^{\prime \prime}\right) ; a: \top-* \neg \top^{*} ; b^{\prime}: \top, c^{\prime}: \top ; b^{\prime \prime}: \top^{*} ; c^{\prime \prime}: \top^{*} \vdash}}{\frac{(b, c \triangleright a) ;\left(b^{\prime}, b \triangleright b^{\prime \prime}\right) ;\left(c^{\prime}, c \triangleright c^{\prime \prime}\right) ; a: \top-* \neg \top^{*} ; b^{\prime}: \top, c^{\prime}: \top \vdash{ }_{-}^{\prime}: \neg \top^{*} ; c^{\prime \prime}: \neg \top^{*}}{(b, c \triangleright a) ; a: \top-* \neg \top^{*} \vdash b: \top-* \neg \top^{*} ; c: \top-* \neg \top^{*}}} L^{2} \\
\frac{(b, c \triangleright a) ; b: \neg\left(\top-* \neg \top^{*}\right) ; c: \neg\left(\top-\neg \top^{*}\right) \vdash a: \neg\left(\top-* \neg \top^{*}\right)}{a: F * F \vdash a: F} R^{2} \\
\frac{a}{\vdash a:(F * F) \rightarrow F} \rightarrow R
\end{gathered}
$$

The correct relational atom that is required to split $a: \top-* \neg \top^{*}$ is $\left(w^{\prime \prime}, a \triangleright \epsilon\right)$. However, in the labelled sequent calculus we can only obtain $w^{\prime \prime}, w^{\prime} \triangleright \epsilon$. Although $w^{\prime}$ and $a$ both have exactly the same children, but the non-deterministic monoid allows the composition $b \circ c$ to be multiple elements, or even $\emptyset$ in $\mathcal{M}$. Thus we cannot conclude that $w^{\prime}=a$. This can be solved by using $P$ to replace $w^{\prime}$ by $a$, then use $E$ to obtain $\left(w^{\prime \prime}, a \triangleright \epsilon\right)$ on the left hand side of the sequent, then the derivation can go through:

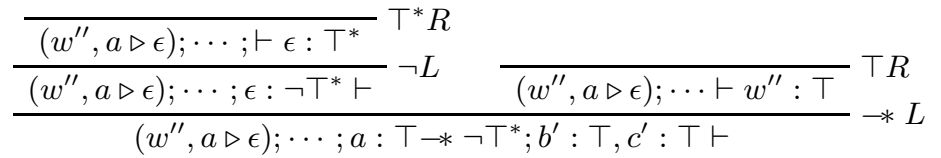

2. The trick to prove $\left(\neg \top^{*}-* \perp\right) \rightarrow \top^{*}$ is to create a relational atom $\left(w, w \triangleright w^{\prime}\right)$, as shown below.

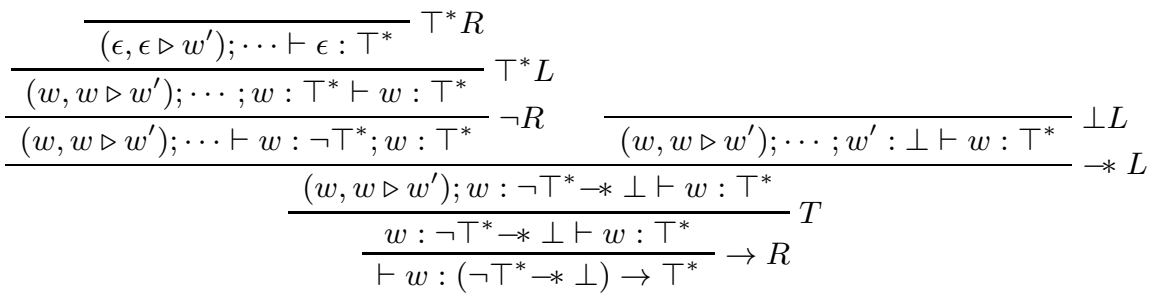

3. The proof for $\left(\top^{*} \wedge((p * q)-* \perp)\right) \rightarrow((p-* \perp) \vee(q-* \perp))$ is as follows.

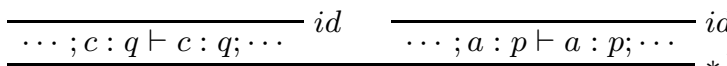

$$
\begin{aligned}
& (a, c \triangleright e) ; \cdots ; a: p ; c: q \vdash e: p * q ; \cdots \quad * R \quad \frac{}{\cdots e: \perp \vdash \cdots} \perp L \\
& \overline{(e, \epsilon \triangleright e) ;(a, c \triangleright e) ;(a, \epsilon \triangleright b) ;(c, \epsilon \triangleright d) ; \epsilon:(p * q)-* \perp ; a: p ; c: q \vdash b: \perp ; d: \perp}-* L \\
& \frac{(a, c \triangleright e) ;(a, \epsilon \triangleright b) ;(c, \epsilon \triangleright d) ; \epsilon:(p * q)-* \perp ; a: p ; c: q \vdash b: \perp ; d: \perp}{T} \\
& \underline{(a, \epsilon \triangleright b) ;(c, \epsilon \triangleright d) ; \epsilon:(p * q)-* \perp ; a: p ; c: q \vdash b: \perp ; d: \perp}{ }_{-* R^{2}} \\
& \epsilon:(p * q)-* \perp \vdash \epsilon: p-* \perp ; \epsilon: q-* \perp \\
& \frac{w: \top^{*} ; w:(p * q)-* \perp \vdash w: p-* \perp ; w: q-* \perp}{w: \top^{*} \wedge((p * q)-* \perp) \vdash w:(p-* \perp) \vee(q-* \perp)} \wedge L ; \vee R
\end{aligned}
$$

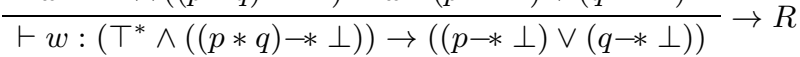

4. The proof for $\neg\left(\top^{*} \wedge A \wedge\left(B * \neg\left(C-*\left(\top^{*} \rightarrow A\right)\right)\right)\right)$ in $L S_{B B I}$ is as follows.

$$
\begin{aligned}
& \overline{(c, b \triangleright \epsilon) ;(a, b \triangleright \epsilon) ; \epsilon: A ; a: B ; c: C \vdash \epsilon: A} i d
\end{aligned}
$$

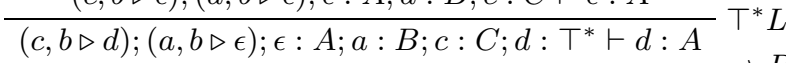

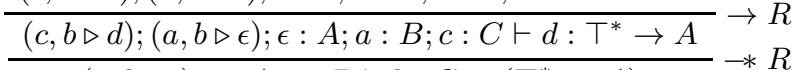

$$
\begin{aligned}
& \frac{(a, b \triangleright \epsilon) ; \epsilon: A ; a: B \vdash b: C-*\left(\top^{*} \rightarrow A\right)}{(a, b \triangleright \epsilon) ; \epsilon: A ; a: B ; b: \neg\left(C-*\left(\top^{*} \rightarrow A\right)\right) \vdash} \neg L \\
& \frac{\overline{(a, b \triangleright \epsilon) ; \epsilon: A ; a: B ; b: \neg\left(C-*\left(\top^{*} \rightarrow A\right)\right) \vdash}}{\epsilon: A ; \epsilon: B * \neg\left(C-*\left(\top^{*} \rightarrow A\right)\right) \vdash} * L
\end{aligned}
$$

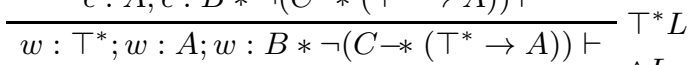

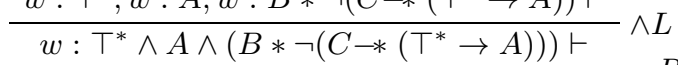

$$
\begin{aligned}
& \overline{\vdash w: \neg\left(\top^{*} \wedge A \wedge\left(B * \neg\left(C-*\left(\top^{*} \rightarrow A\right)\right)\right)\right)} \neg R
\end{aligned}
$$

